\title{
Selected Topics in Microwave Instabilities and Linacs
}

\author{
K.Y. Ng
}

Fermilab

June, 2013

Slides can be downloaded from www-ap.fnal.gov/ng/sinap-leture.pdf

This file is frequently updated. Two excercises can be downloaded from www-ap.fnal.gov/ng/exercises.pdf 
FERMILAB-FN-0961-APC

\title{
Selected Topics in Microwave Instabilities and Linacs
}

\author{
K.Y. Ng \\ Fermilab, Batavia, IL 60510
}

(June, 2013)

\begin{abstract}
The Shanghai Institute of Applied Physics (SINAP) is embarking on its first X-ray free-electron laser (FEL) project. It is a cascading high-gain harmonic generation FEL. Microwave instabilities driven by various effects, especially the space-charge force, will degrade the quality of the electron beam before entering into the undulator. However, inside the undulator, the occurrence of microbunching becomes an utmost important ingredient for the generation of coherent radiation. In short, controlled and uncontrolled microwave instabilities must be fully understood in such a project. These are the slides of a series of eight-hour lectures given at the SINAP in June of 2013, with the intention of a fully understanding of the microbunching phenomenon. The sections of wake field and impedance theory are added as an introduction for those who are not familiar with the subject.
\end{abstract}

This manuscript has been authored by Fermi Research Alliance, LLC under Contract No. DE-AC02-07CH11359 with the U.S. Department of Energy, Office of Science, Office of High Energy Physics. 


\section{Contents}

(1) Wake Functions and Coupling Impedances

(2) Coupling Impedances

(3) Landau Damping

(4) Microwave Instabilities

(5) Transition Growth From Noise

(6) FEL and Micro-bunching

(7) Beam Breakup

(8) References 


\section{Introduction}

- A particle interacts with the vacuum chamber produces EM fields.

- The motion of a particle following is perturbed.

$$
\begin{array}{r}
(\vec{E}, \vec{B})_{\text {seen by barticle }} \\
\text { pale }
\end{array}
$$

where

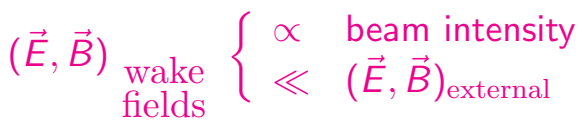




\section{Introduction}

- A particle interacts with the vacuum chamber produces EM fields.

- The motion of a particle following is perturbed.

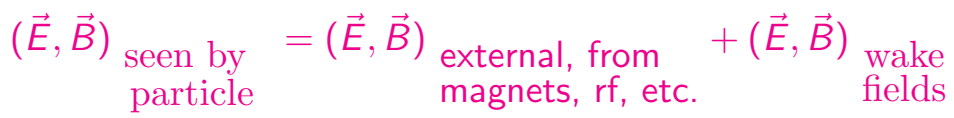

where

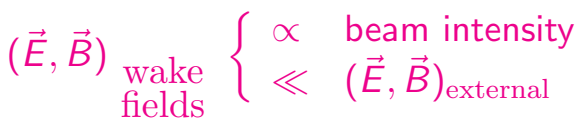

- Perturbation breaks down when potential-well distortion is large. Then, distortion has to be included into non-perturbative part. 


\section{Introduction}

- A particle interacts with the vacuum chamber produces EM fields.

- The motion of a particle following is perturbed.

$$
\begin{array}{r}
(\vec{E}, \vec{B})_{\text {seen by }} \\
\text { particle }
\end{array}=(\vec{E}, \vec{B})_{\begin{array}{l}
\text { external, from } \\
\text { magnets, rf, etc. }
\end{array}}+(\vec{E}, \vec{B})_{\text {wake }}^{\text {fields }}
$$

where

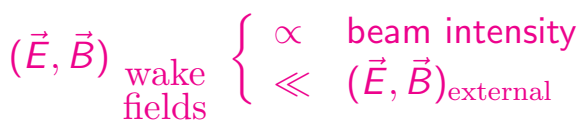

- Perturbation breaks down when potential-well distortion is large. Then, distortion has to be included into non-perturbative part.

- What we need to compute are the EM wake fields at a distance $z$ behind the source particle.

- The computation of the wake fields is nontrivial.

- Two approximations lead to a lot of simplification. 


\section{Rigid-Bunch Approximation [1]}

- Motion of beam not affected during traversal through discontinuities.

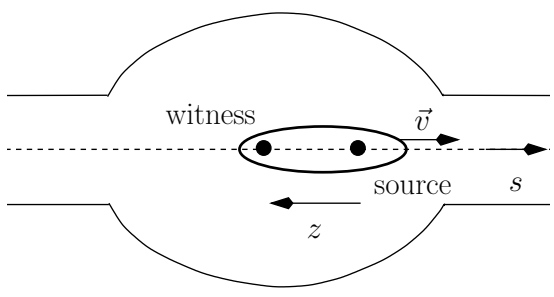

Source particle at $s=\beta c t$

Witness particle at $s=z+\beta c t$

$z<0$ for particle following.

This does not imply no syn. motion. Just require $z$ and $\beta c$ do not change after traversing the discontinuity.

- Rigidity implies beam at high energies. 


\section{Rigid-Bunch Approximation [1]}

- Motion of beam not affected during traversal through discontinuities.

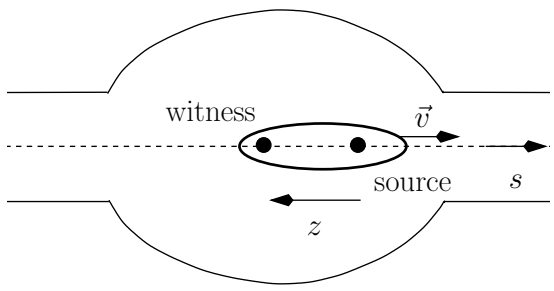

Source particle at $s=\beta c t$

Witness particle at $s=z+\beta c t$

$z<0$ for particle following.

This does not imply no syn. motion. Just require $z$ and $\beta c$ do not change after traversing the discontinuity.

- Rigidity implies beam at high energies.

\section{Impulse Approximation}

- We do not care about the wake fields $\vec{E}, \vec{B}$, or the wake force $\vec{F}$.

- We only care about the impulse

$$
\Delta \vec{p}=\int_{-\infty}^{\infty} d t \vec{F}=\int_{-\infty}^{\infty} d t q(\vec{E}+\vec{v} \times \vec{B}) \quad q \text { is charge of witness particle }
$$

- We will see how the simplification evolves. 


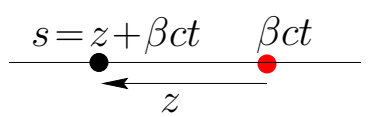

- Maxwell equation for witness particle at $(x, y, s, t)$ with $s=z+\beta c t$ : and $z$ constant

$$
\begin{aligned}
\vec{\nabla} \cdot \vec{E} & =\frac{\rho}{\epsilon_{0}} \\
\vec{\nabla} \times \vec{B}-\frac{1}{c^{2}} \frac{\partial \vec{E}}{\partial t} & =\mu_{0} \beta c \rho \hat{s} \\
\vec{\nabla} \cdot \vec{B} & =0 \\
\vec{\nabla} \times \vec{E}+\frac{\partial \vec{B}}{\partial t} & =0
\end{aligned}
$$

Gauss's law for electric charge

Ampere's law

Gauss's law for magnetic charge

Faraday's \& Lenz law

- Want to write Maxwell equation for the impulse $\Delta \vec{p}(x, y, z, t)$.

First compute

with $\vec{F}(x, y, z, t)=q(\vec{E}+\vec{v} \times \vec{B})$

$$
\begin{aligned}
\vec{\nabla} \cdot \vec{F} & =\frac{q \rho}{\epsilon_{0} \gamma^{2}}-\frac{q \beta}{c} \frac{\partial E_{s}}{\partial t}, \\
\vec{\nabla} \times \vec{F} & =-q\left(\frac{\partial}{\partial t}+\beta c \frac{\partial}{\partial s}\right) \vec{B} .
\end{aligned}
$$




\section{The Wake Force Equations}

- Wake force: $\vec{F}(x, y, z, t)=q(\vec{E}+\vec{v} \times \vec{B})$

- Maxwell's equations: $\vec{\nabla} \cdot \vec{E}=\frac{\rho}{\epsilon_{0}}, \quad \vec{\nabla} \cdot \vec{B}=0$,

- Divergent:

$$
\vec{\nabla} \times \vec{E}=-\frac{\partial \vec{B}}{\partial t}, \quad \vec{\nabla} \times \vec{B}=\frac{1}{c^{2}} \frac{\partial \vec{E}}{\partial t}+\mu_{0} \beta c \rho \hat{s}
$$

$$
\begin{aligned}
& \vec{\nabla} \cdot \vec{F}=q(\vec{\nabla} \cdot \vec{E}+\vec{\nabla} \cdot \vec{v} \times \vec{B})=\frac{q \rho}{\epsilon_{0}}-q \vec{v}\left(\frac{1}{c^{2}} \frac{\partial \vec{E}}{\partial t}+\mu_{0} \beta c \rho \hat{s}\right) \\
& =\frac{q \rho}{\epsilon_{0} \gamma^{2}}-\frac{q \beta}{c} \frac{\partial E_{s}}{\partial t} \quad c=\frac{1}{\sqrt{\epsilon_{0} \mu_{0}}}
\end{aligned}
$$

- Curl:

$$
\begin{aligned}
& \vec{\nabla} \times \vec{F}=q \vec{\nabla} \times \vec{E}+q \vec{\nabla} \times(\vec{v} \times \vec{B}) \\
& =-q \frac{\partial \vec{B}}{\partial t}+q \vec{v}(\vec{\nabla} \cdot \vec{B})-q v \frac{\partial \vec{B}}{\partial s}=q\left(\frac{\partial}{\partial t}+v \frac{\partial}{\partial s}\right) \vec{B}=q \frac{d \vec{B}}{d t}
\end{aligned}
$$




$$
\begin{aligned}
& \vec{\nabla} \times \Delta \vec{p}(x, y, z)=\int_{-\infty}^{\infty} d t[\vec{\nabla} \times \vec{F}(x, y, s, t)]_{s=z+\beta c t} . \\
& \text { this } \vec{\nabla} \text { refers } \\
& \text { this } \vec{\nabla} \text { refers } \\
& \text { to } x, y, z \\
& \text { to } x, y, s
\end{aligned}
$$




$$
\begin{aligned}
& \vec{\nabla} \times \Delta \vec{p}(x, y, z)=\int_{-\infty}^{\infty} d t\left[\begin{array}{l}
\vec{\nabla} \times \vec{F} \\
\uparrow
\end{array}(x, y, s, t)\right]_{s=z+\beta c t} \\
& \text { this } \vec{\nabla} \text { refers } \\
& \text { to } x, y, z \\
& \text { this } \vec{\nabla} \text { refers } \\
& \text { to } x, y, s
\end{aligned}
$$

\section{We obtain}

$$
\begin{aligned}
\vec{\nabla} \times \Delta \vec{p} & =-q \int_{-\infty}^{\infty} d t\left[\left(\frac{\partial}{\partial t}+\beta c \frac{\partial}{\partial s}\right) \vec{B}(x, y, s, t)\right]_{s=z+\beta c t} \\
& =-q \int_{-\infty}^{\infty} d t \frac{d \vec{B}}{d t}=-\left.q \vec{B}(x, y, z+\beta c t, t)\right|_{t=-\infty} ^{\infty}=0
\end{aligned}
$$




$$
\begin{aligned}
& \left.\underset{\uparrow}{\vec{\nabla}} \times \Delta \vec{p}(x, y, z)=\int_{-\infty}^{\infty} d t \underset{\uparrow}{[\vec{\nabla} \times \vec{F}}(x, y, s, t)\right]_{s=z+\beta c t} . \\
& \text { this } \vec{\nabla} \text { refers } \\
& \text { to } x, y, z \\
& \text { this } \vec{\nabla} \text { refers } \\
& \text { to } x, y, s
\end{aligned}
$$

We obtain

$$
\begin{aligned}
\vec{\nabla} \times \Delta \vec{p} & =-q \int_{-\infty}^{\infty} d t\left[\left(\frac{\partial}{\partial t}+\beta c \frac{\partial}{\partial s}\right) \vec{B}(x, y, s, t)\right]_{s=z+\beta c t} \\
& =-q \int_{-\infty}^{\infty} d t \frac{d \vec{B}}{d t}=-\left.q \vec{B}(x, y, z+\beta c t, t)\right|_{t=-\infty} ^{\infty}=0
\end{aligned}
$$

- Dot product with $\hat{s} \Longrightarrow \hat{s} \cdot(\vec{\nabla} \times \Delta \vec{p})=0 \Longrightarrow \frac{\partial \Delta p_{x}}{\partial y}=\frac{\partial \Delta p_{y}}{\partial x}$ 


$$
\begin{aligned}
& \left.\underset{\uparrow}{\vec{\nabla}} \times \Delta \vec{p}(x, y, z)=\int_{-\infty}^{\infty} d t \underset{\uparrow}{[\vec{\nabla} \times \vec{F}}(x, y, s, t)\right]_{s=z+\beta c t} . \\
& \text { this } \vec{\nabla} \text { refers } \\
& \text { to } x, y, z \\
& \text { this } \vec{\nabla} \text { refers } \\
& \text { to } x, y, s
\end{aligned}
$$

We obtain

$$
\begin{aligned}
\vec{\nabla} \times \Delta \vec{p} & =-q \int_{-\infty}^{\infty} d t\left[\left(\frac{\partial}{\partial t}+\beta c \frac{\partial}{\partial s}\right) \vec{B}(x, y, s, t)\right]_{s=z+\beta c t} \\
& =-q \int_{-\infty}^{\infty} d t \frac{d \vec{B}}{d t}=-\left.q \vec{B}(x, y, z+\beta c t, t)\right|_{t=-\infty} ^{\infty}=0
\end{aligned}
$$

- Dot product with $\hat{s} \Longrightarrow \hat{s} \cdot(\vec{\nabla} \times \Delta \vec{p})=0 \Longrightarrow \frac{\partial \Delta p_{x}}{\partial y}=\frac{\partial \Delta p_{y}}{\partial x}$

- Cross product with $\hat{s} \Longrightarrow \frac{\partial}{\partial z} \Delta \vec{p}_{\perp}=\vec{\nabla}_{\perp} \Delta p_{s} \quad \longleftarrow$ P-W Theorem 


$$
\begin{aligned}
& \underset{\uparrow}{\vec{\nabla}} \times \Delta \vec{p}(x, y, z)=\int_{-\infty}^{\infty} d t \underset{\uparrow}{[\vec{\nabla} \times \vec{F}(x, y, s, t)]_{s=z+\beta c t} .} \\
& \text { this } \vec{\nabla} \text { refers } \\
& \text { to } x, y, z \\
& \text { this } \vec{\nabla} \text { refers } \\
& \text { to } x, y, s
\end{aligned}
$$

We obtain

$$
\begin{aligned}
\vec{\nabla} \times \Delta \vec{p} & =-q \int_{-\infty}^{\infty} d t\left[\left(\frac{\partial}{\partial t}+\beta c \frac{\partial}{\partial s}\right) \vec{B}(x, y, s, t)\right]_{s=z+\beta c t} \\
& =-q \int_{-\infty}^{\infty} d t \frac{d \vec{B}}{d t}=-\left.q \vec{B}(x, y, z+\beta c t, t)\right|_{t=-\infty} ^{\infty}=0
\end{aligned}
$$

- Dot product with $\hat{s} \Longrightarrow \hat{s} \cdot(\vec{\nabla} \times \Delta \vec{p})=0 \Longrightarrow \frac{\partial \Delta p_{x}}{\partial y}=\frac{\partial \Delta p_{y}}{\partial x}$

- Cross product with $\hat{s} \Longrightarrow \frac{\partial}{\partial z} \Delta \vec{p}_{\perp}=\vec{\nabla}_{\perp} \Delta p_{s} \quad \longleftarrow$ P-W Theorem

- P-W theorem gives strong restriction between long. and trans.

- But it is very general. Does not depend on any boundary conditions. Even do not require $\beta=1$. 


\section{Supplement to Panofsky-Wenzel Theorem}

$$
\beta=1 \Longrightarrow \vec{\nabla}_{\perp} \cdot \Delta \vec{p}_{\perp}=0 .
$$

Proof:

$$
\begin{aligned}
\vec{\nabla} \cdot \Delta \vec{p} & =\int_{-\infty}^{\infty} d t[\vec{\nabla} \cdot \vec{F}(x, y, s, t)]_{s=z+c t}=q \int_{-\infty}^{\infty} d t\left[\frac{\rho}{\epsilon_{0} \gamma^{2}}-\frac{\beta}{c} \frac{\partial E_{s}}{\partial t}\right]_{s=z+c t} \\
& \stackrel{\gamma \rightarrow \infty}{\longrightarrow} q \int_{-\infty}^{\infty} d t\left[\frac{\partial E_{s}}{\partial s}\right]_{s=z+c t}=\frac{\partial}{\partial z} \Delta p_{s}
\end{aligned}
$$

Use has been made of

- Space-charge term $\frac{q \rho}{\epsilon_{0} \gamma^{2}}$ omitted because $\beta \rightarrow 1$.

$$
\text { (2) } \frac{\partial}{\partial t} E_{s}(s, t)=\frac{d}{d t} E_{s}(s, t)-\frac{d s}{d t} \frac{\partial}{\partial s} E_{s}(s, t) \text {. }
$$

Maxwell equations now become

$$
\vec{\nabla} \times \Delta \vec{p}=0 \text { and } \vec{\nabla} \cdot \Delta \vec{p}=\frac{\partial}{\partial z} \Delta p_{s}
$$

without any source terms. 


\section{Cylindrical Symmetric Vacuum Chamber}

$$
\left\{\begin{array} { r l } 
{ \frac { \partial } { \partial r } ( r \Delta p _ { \theta } ) } & { = \frac { \partial } { \partial \theta } \Delta p _ { r } } \\
{ \frac { \partial } { \partial z } \Delta p _ { r } } & { = \frac { \partial } { \partial r } \Delta p _ { s } } \\
{ \frac { \partial } { \partial z } \Delta p _ { \theta } } & { = \frac { 1 } { r } \frac { \partial } { \partial \theta } \Delta p _ { s } } \\
{ \frac { \partial } { \partial r } ( r \Delta p _ { r } ) } & { = - \frac { \partial } { \partial \theta } \Delta p _ { \theta } \quad ( \beta = 1 ) }
\end{array} \quad \Longrightarrow \left\{\begin{array}{rl}
\frac{\partial}{\partial r}\left(r \Delta \tilde{p}_{\theta}\right) & =-m \Delta \tilde{p}_{r} \\
\frac{\partial}{\partial z} \Delta \tilde{p}_{r} & =\frac{\partial}{\partial r} \Delta \tilde{p}_{s} \\
\frac{\partial}{\partial z} \Delta \tilde{p}_{\theta} & =-\frac{m}{r} \Delta \tilde{p}_{s} \\
\frac{\partial}{\partial r}\left(r \Delta \tilde{p}_{r}\right) & =-m \Delta \tilde{p}_{\theta} \quad(\beta=1)
\end{array}\right.\right.
$$

- Cylindrical symmetry $\Longrightarrow$ expansion in terms of $\cos m \theta$ or $\sin m \theta$.

We write $\Delta p_{s}=\Delta \tilde{p}_{s} \cos m \theta, \quad \Delta p_{r}=\Delta \tilde{p}_{r} \cos m \theta, \quad \Delta p_{\theta}=\Delta \tilde{p}_{\theta} \sin m \theta$, where $\Delta \tilde{p}_{s}, \Delta \tilde{p}_{r}$, and $\Delta \tilde{p}_{\theta}$ are $\theta$-independent. 


\section{Cylindrical Symmetric Vacuum Chamber}

$$
\left\{\begin{array} { r l } 
{ \frac { \partial } { \partial r } ( r \Delta p _ { \theta } ) } & { = \frac { \partial } { \partial \theta } \Delta p _ { r } } \\
{ \frac { \partial } { \partial z } \Delta p _ { r } } & { = \frac { \partial } { \partial r } \Delta p _ { s } } \\
{ \frac { \partial } { \partial z } \Delta p _ { \theta } } & { = \frac { 1 } { r } \frac { \partial } { \partial \theta } \Delta p _ { s } } \\
{ \frac { \partial } { \partial r } ( r \Delta p _ { r } ) } & { = - \frac { \partial } { \partial \theta } \Delta p _ { \theta } \quad ( \beta = 1 ) }
\end{array} \quad \Longrightarrow \left\{\begin{array}{rl}
\frac{\partial}{\partial r}\left(r \Delta \tilde{p}_{\theta}\right) & =-m \Delta \tilde{p}_{r} \\
\frac{\partial}{\partial z} \Delta \tilde{p}_{r} & =\frac{\partial}{\partial r} \Delta \tilde{p}_{s} \\
\frac{\partial}{\partial z} \Delta \tilde{p}_{\theta} & =-\frac{m}{r} \Delta \tilde{p}_{s} \\
\frac{\partial}{\partial r}\left(r \Delta \tilde{p}_{r}\right) & =-m \Delta \tilde{p}_{\theta} \quad(\beta=1)
\end{array}\right.\right.
$$

- Cylindrical symmetry $\Longrightarrow$ expansion in terms of $\cos m \theta$ or $\sin m \theta$.

We write $\Delta p_{s}=\Delta \tilde{p}_{s} \cos m \theta, \quad \Delta p_{r}=\Delta \tilde{p}_{r} \cos m \theta, \quad \Delta p_{\theta}=\Delta \tilde{p}_{\theta} \sin m \theta$, where $\Delta \tilde{p}_{s}, \Delta \tilde{p}_{r}$, and $\Delta \tilde{p}_{\theta}$ are $\theta$-independent.

- For $m=0, \Delta \tilde{p}_{r}=\Delta \tilde{p}_{\theta}=0$, otherwise $\propto \frac{1}{r}$, singular at $r=0, \therefore$ only $p_{s}$ 


\section{Cylindrical Symmetric Vacuum Chamber}

$$
\left\{\begin{array} { r l } 
{ \frac { \partial } { \partial r } ( r \Delta p _ { \theta } ) } & { = \frac { \partial } { \partial \theta } \Delta p _ { r } } \\
{ \frac { \partial } { \partial z } \Delta p _ { r } } & { = \frac { \partial } { \partial r } \Delta p _ { s } } \\
{ \frac { \partial } { \partial z } \Delta p _ { \theta } } & { = \frac { 1 } { r } \frac { \partial } { \partial \theta } \Delta p _ { s } } \\
{ \frac { \partial } { \partial r } ( r \Delta p _ { r } ) } & { = - \frac { \partial } { \partial \theta } \Delta p _ { \theta } \quad ( \beta = 1 ) }
\end{array} \quad \Longrightarrow \left\{\begin{array}{rl}
\frac{\partial}{\partial r}\left(r \Delta \tilde{p}_{\theta}\right) & =-m \Delta \tilde{p}_{r} \\
\frac{\partial}{\partial z} \Delta \tilde{p}_{r} & =\frac{\partial}{\partial r} \Delta \tilde{p}_{s} \\
\frac{\partial}{\partial z} \Delta \tilde{p}_{\theta} & =-\frac{m}{r} \Delta \tilde{p}_{s} \\
\frac{\partial}{\partial r}\left(r \Delta \tilde{p}_{r}\right) & =-m \Delta \tilde{p}_{\theta} \quad(\beta=1)
\end{array}\right.\right.
$$

- Cylindrical symmetry $\Longrightarrow$ expansion in terms of $\cos m \theta$ or $\sin m \theta$.

We write $\Delta p_{s}=\Delta \tilde{p}_{s} \cos m \theta, \quad \Delta p_{r}=\Delta \tilde{p}_{r} \cos m \theta, \quad \Delta p_{\theta}=\Delta \tilde{p}_{\theta} \sin m \theta$, where $\Delta \tilde{p}_{s}, \Delta \tilde{p}_{r}$, and $\Delta \tilde{p}_{\theta}$ are $\theta$-independent.

- For $m=0, \Delta \tilde{p}_{r}=\Delta \tilde{p}_{\theta}=0$, otherwise $\propto \frac{1}{r}$, singular at $r=0, \therefore$ only $p_{s}$

- For $m \neq 0, \frac{\partial}{\partial r}\left[r \frac{\partial}{\partial r}\left(r \Delta \tilde{p}_{r}\right)\right]=m^{2} \Delta \tilde{p}_{r} \Longrightarrow \Delta p_{r}(r, \theta, z) \sim m r^{m-1} \cos m \theta$ 


\section{Definition of Wake Functions}

- Formal solution can be written as

$$
\begin{aligned}
& \left\{\begin{array}{ll|}
v \Delta \vec{p}_{\perp}=-q \mathcal{Q}_{m} W_{m}(z) m r^{m-1}(\hat{r} \cos m \theta-\hat{\theta} \sin m \theta) & \begin{array}{l}
\text { dimension is } \\
\text { energy }
\end{array} \\
v \Delta p_{s}=-q \mathcal{Q}_{m} W_{m}^{\prime}(z) r^{m} \cos m \theta & \longrightarrow \text { transverse wake function of azimuthal } m
\end{array}\right. \\
& \text { - Defn: }\left\{\begin{array}{l}
W_{m}(z) \longrightarrow \text { longitudinal wake function of azimuthal } m \\
W_{m}^{\prime}(z) \longrightarrow
\end{array}\right.
\end{aligned}
$$




\section{Definition of Wake Functions}

- Formal solution can be written as

$\left\{\begin{aligned} v \Delta \vec{p}_{\perp} & =-q \mathcal{Q}_{m} W_{m}(z) m r^{m-1}(\hat{r} \cos m \theta-\hat{\theta} \sin m \theta) \\ v \Delta p_{s} & =-q \mathcal{Q}_{m} W_{m}^{\prime}(z) r^{m} \cos m \theta\end{aligned}\right.$

dimension is energy

- Defn: $\left\{\begin{array}{l}W_{m}(z) \longrightarrow \text { transverse wake function of azimuthal } m \\ W_{m}^{\prime}(z) \longrightarrow \text { longitudinal wake function of azimuthal } m\end{array}\right.$

They are functions of $z$ only and dependent on boundary conditions. They are related because of P-W theorem. 


\section{Definition of Wake Functions}

- Formal solution can be written as

$\left\{\begin{aligned} v \Delta \vec{p}_{\perp} & =-q \mathcal{Q}_{m} W_{m}(z) m r^{m-1}(\hat{r} \cos m \theta-\hat{\theta} \sin m \theta) \\ v \Delta p_{s} & =-q \mathcal{Q}_{m} W_{m}^{\prime}(z) r^{m} \cos m \theta\end{aligned}\right.$

dimension is energy

- Defn: $\left\{\begin{array}{l}W_{m}(z) \longrightarrow \text { transverse wake function of azimuthal } m \\ W_{m}^{\prime}(z) \longrightarrow \text { longitudinal wake function of azimuthal } m\end{array}\right.$

They are functions of $z$ only and dependent on boundary conditions.

They are related because of P-W theorem.

- $\mathcal{Q}_{m}=e a^{m}$ is $m$ th multipole of source particle of charge $e$. $W_{m}(z)$ has dimension $\mathrm{V} /$ Coulomb $/ \mathrm{m}^{2 m-1}$. 


\section{Definition of Wake Functions}

- Formal solution can be written as

$\left\{\begin{aligned} v \Delta \vec{p}_{\perp} & =-q \mathcal{Q}_{m} W_{m}(z) m r^{m-1}(\hat{r} \cos m \theta-\hat{\theta} \sin m \theta) \\ v \Delta p_{s} & =-q \mathcal{Q}_{m} W_{m}^{\prime}(z) r^{m} \cos m \theta\end{aligned}\right.$

dimension is energy

- Defn: $\left\{\begin{array}{l}W_{m}(z) \longrightarrow \text { transverse wake function of azimuthal } m \\ W_{m}^{\prime}(z) \longrightarrow \text { longitudinal wake function of azimuthal } m\end{array}\right.$

They are functions of $z$ only and dependent on boundary conditions.

They are related because of P-W theorem.

- $\mathcal{Q}_{m}=e a^{m}$ is $m$ th multipole of source particle of charge $e$. $W_{m}(z)$ has dimension $\mathrm{V} /$ Coulomb $/ \mathrm{m}^{2 m-1}$.

- Recall that solution of $\vec{E}$ and $\vec{B}$ reduces to solution of $W_{m}(z)$ only. Simplification comes from $\mathrm{P}-\mathrm{W}$ theorem or rigid-bunch and impulse approximations.

- negative sign in front is a convention to make $W_{m}^{\prime}(z)>0$, since witness particle loses energy from impulse. 


\section{Some Properties of Wake Functions}
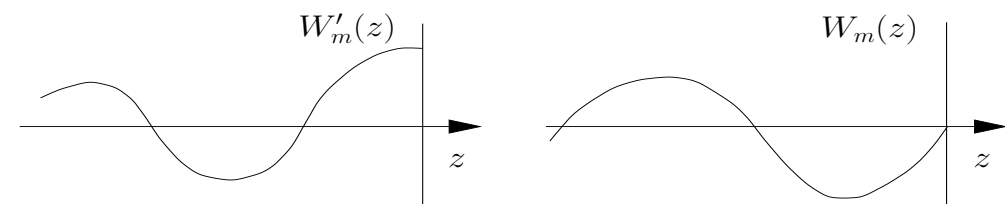


\section{Some Properties of Wake Functions}
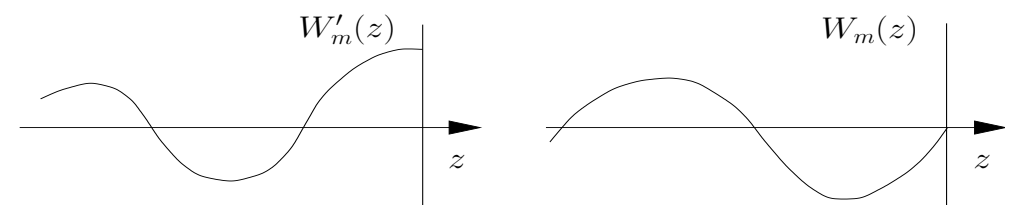

Fundamental Theorem of Beam Loading (P. Wilson)

A particle sees half of its wake, or $\frac{1}{2} W_{m}^{\prime}\left(0_{-}\right)$. 


\section{Some Properties of Wake Functions}
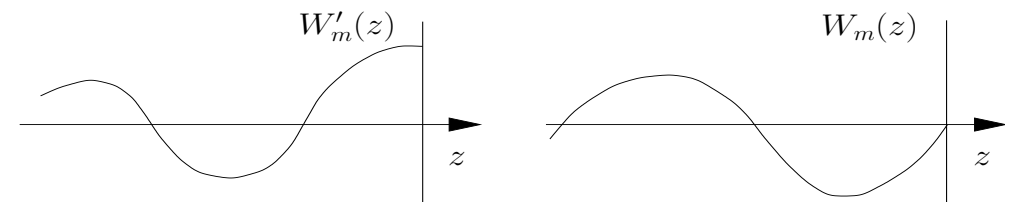

Fundamental Theorem of Beam Loading (P. Wilson)

A particle sees half of its wake, or $\frac{1}{2} W_{m}^{\prime}\left(0_{-}\right)$.

Proof:

A particle of charge $q$ passes a thin lossless cavity, excites cavity.

Energy gained $\Delta \mathcal{E}_{1}=-f q^{2} W_{m}^{\prime}\left(0_{-}\right)$, i.e., sees fraction $f$ of own wake. 


\section{Some Properties of Wake Functions}
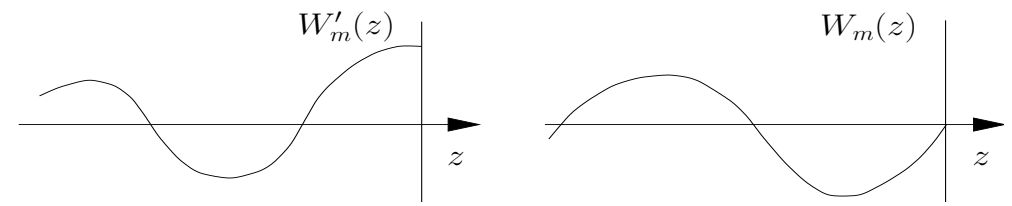

Fundamental Theorem of Beam Loading (P. Wilson)

A particle sees half of its wake, or $\frac{1}{2} W_{m}^{\prime}\left(0_{-}\right)$.

Proof:

A particle of charge $q$ passes a thin lossless cavity, excites cavity. Energy gained $\Delta \mathcal{E}_{1}=-f q^{2} W_{m}^{\prime}\left(0_{-}\right)$, i.e., sees fraction $f$ of own wake. Half cycle later, a 2nd particle of same charge passes the cavity.

Energy gained $\Delta \mathcal{E}_{2}=-f q^{2} W_{m}^{\prime}\left(0_{-}\right)+q^{2} W_{m}^{\prime}\left(0_{-}\right)$. 


\section{Some Properties of Wake Functions}
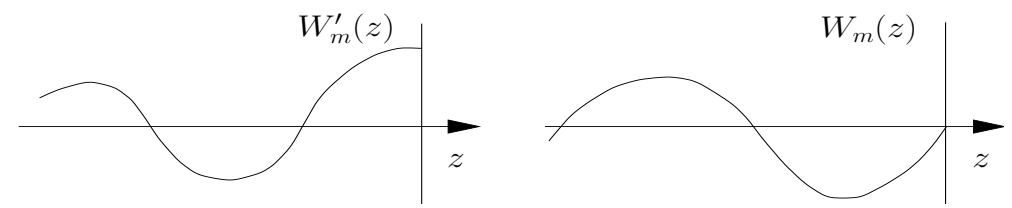

\section{Fundamental Theorem of Beam Loading (P. Wilson)}

A particle sees half of its wake, or $\frac{1}{2} W_{m}^{\prime}\left(0_{-}\right)$.

\section{Proof:}

A particle of charge $q$ passes a thin lossless cavity, excites cavity.

Energy gained $\Delta \mathcal{E}_{1}=-f q^{2} W_{m}^{\prime}\left(0_{-}\right)$, i.e., sees fraction $f$ of own wake.

Half cycle later, a 2nd particle of same charge passes the cavity.

Energy gained $\Delta \mathcal{E}_{2}=-f q^{2} W_{m}^{\prime}\left(0_{-}\right)+q^{2} W_{m}^{\prime}\left(0_{-}\right)$.

Field inside cavity is completely cancelled.

$\Delta \mathcal{E}_{1}+\Delta \mathcal{E}_{2}=-2 f q^{2} W_{m}^{\prime}\left(0_{-}\right)+q^{2} W_{m}^{\prime}\left(0_{-}\right)=0 \Longrightarrow f=\frac{1}{2}$. 


\section{Properties of Wake Functions}

- $W_{m}^{\prime}(z)=0$ for $z>0$.

- $W_{m}^{\prime}\left(0_{-}\right) \geq 0$

\section{(causality)}

(energy conservation) 


\section{Properties of Wake Functions}

- $W_{m}^{\prime}(z)=0$ for $z>0$.

- $W_{m}^{\prime}\left(0_{-}\right) \geq 0$

- $\left|W_{m}^{\prime}(-z)\right| \leq W_{m}^{\prime}\left(0_{-}\right)$.

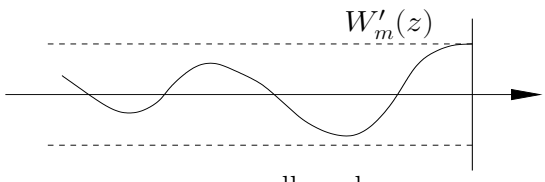

allowed

\section{(causality)}

(energy conservation) 


\section{Properties of Wake Functions}

- $W_{m}^{\prime}(z)=0$ for $z>0$.

(causality)

- $W_{m}^{\prime}\left(0_{-}\right) \geq 0$

(energy conservation)

- $\left|W_{m}^{\prime}(-z)\right| \leq W_{m}^{\prime}\left(0_{-}\right)$.

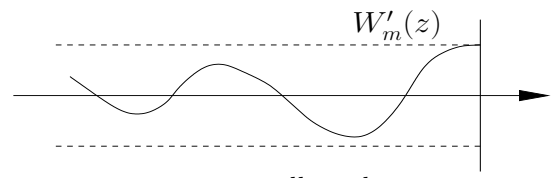

allowed

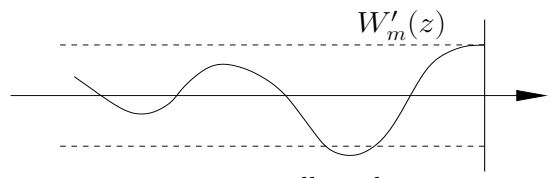

not allowed

$P$

1st particle of charge $q$ loses energy $\frac{1}{2} q^{2} W^{\prime}\left(0_{-}\right)$.

2nd particle of charge $q$ loses energy $\frac{1}{2} q^{2} W^{\prime}\left(0_{-}\right)+q^{2} W_{0}^{\prime}(-z)$.

Total loss $q^{2} W^{\prime}\left(0_{-}\right)+q^{2} W_{0}^{\prime}(-z) \geq 0$. Or $-W_{0}^{\prime}(-z) \leq W_{0}^{\prime}\left(0_{-}\right)$. 


\section{Properties of Wake Functions}

- $W_{m}^{\prime}(z)=0$ for $z>0$.

(causality)

- $W_{m}^{\prime}\left(0_{-}\right) \geq 0$

(energy conservation)

- $\left|W_{m}^{\prime}(-z)\right| \leq W_{m}^{\prime}\left(0_{-}\right)$.

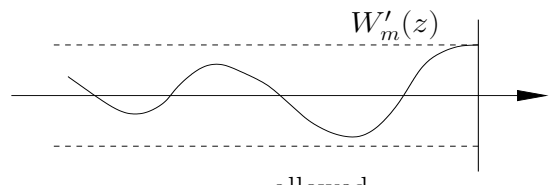

allowed

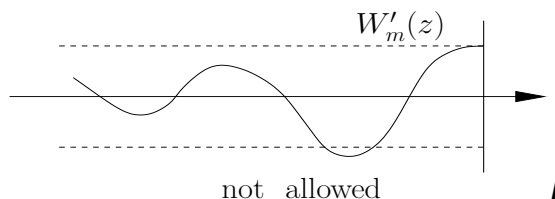

not allowed

$P_{I}$

1st particle of charge $q$ loses energy $\frac{1}{2} q^{2} W^{\prime}\left(0_{-}\right)$.

2nd particle of charge $q$ loses energy $\frac{1}{2} q^{2} W^{\prime}\left(0_{-}\right)+q^{2} W_{0}^{\prime}(-z)$.

Total loss $q^{2} W^{\prime}\left(0_{-}\right)+q^{2} W_{0}^{\prime}(-z) \geq 0$. Or $-W_{0}^{\prime}(-z) \leq W_{0}^{\prime}\left(0_{-}\right)$.

2nd particle of charge $-q$ loses energy $\frac{1}{2} q^{2} W^{\prime}\left(0_{-}\right)-q^{2} W_{0}^{\prime}(-z)$.

Total loss $q^{2} W^{\prime}\left(0_{-}\right)-q^{2} W_{0}^{\prime}(-z) \geq 0$. Or $W_{0}^{\prime}(-z) \leq W_{0}^{\prime}\left(0_{-}\right)$. 


\section{Properties of Wake Functions (cont.)}

- $W_{m}^{\prime}(-D)=W_{m}^{\prime}\left(0_{-}\right)$for some $D>0 \Longrightarrow$ wake is of period $D$.

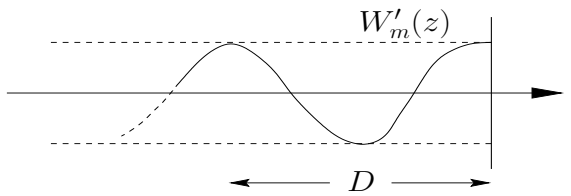




\section{Properties of Wake Functions (cont.)}

- $W_{m}^{\prime}(-D)=W_{m}^{\prime}\left(0_{-}\right)$for some $D>0 \Longrightarrow$ wake is of period $D$.

Proof:

Energy loss:

1. $\frac{1}{2} q_{1}^{2} W_{0}^{\prime}\left(0_{-}\right)$.

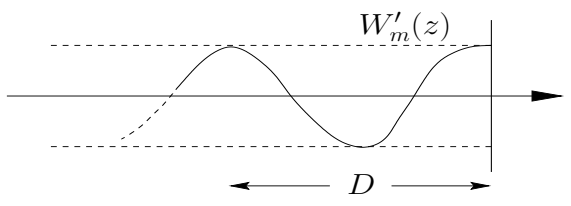

2. $\frac{1}{2} q_{2}^{2} W_{0}^{\prime}\left(0_{-}\right)+q_{1} q_{2} W_{0}^{\prime}(-z)$.

3. $\frac{1}{2} q_{2}^{2} W_{0}^{\prime}\left(0_{-}\right)-q_{1} q_{2} W_{0}^{\prime}(-z-D)-q_{2}^{2} W_{0}^{\prime}(-D)$.

Since total must be $\geq 0$ and $q_{1}$ arbitrary, $W_{0}^{\prime}(-z) \geq W_{0}^{\prime}(-z-D)$.

Change 3 charges to $\left(q_{1},-q_{2}, q_{2}\right)$ to get $W_{0}^{\prime}(-z) \leq W_{0}^{\prime}(-z-D)$.

- Area under $W_{m}^{\prime}(z)$ is non-negative. 


\section{Properties of Wake Functions (cont.)}

- $W_{m}^{\prime}(-D)=W_{m}^{\prime}\left(0_{-}\right)$for some $D>0 \Longrightarrow$ wake is of period $D$.

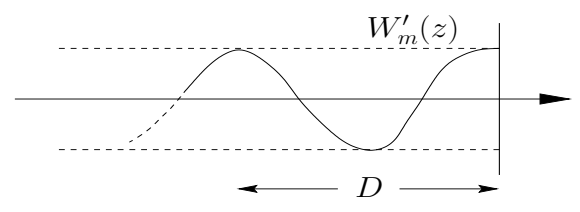

Proof:

Energy loss:

1. $\frac{1}{2} q_{1}^{2} W_{0}^{\prime}\left(0_{-}\right)$.

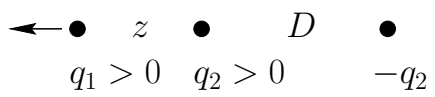

2. $\frac{1}{2} q_{2}^{2} W_{0}^{\prime}\left(0_{-}\right)+q_{1} q_{2} W_{0}^{\prime}(-z)$.

3. $\frac{1}{2} q_{2}^{2} W_{0}^{\prime}\left(0_{-}\right)-q_{1} q_{2} W_{0}^{\prime}(-z-D)-q_{2}^{2} W_{0}^{\prime}(-D)$.

Since total must be $\geq 0$ and $q_{1}$ arbitrary, $W_{0}^{\prime}(-z) \geq W_{0}^{\prime}(-z-D)$.

Change 3 charges to $\left(q_{1},-q_{2}, q_{2}\right)$ to get $W_{0}^{\prime}(-z) \leq W_{0}^{\prime}(-z-D)$.

- Area under $W_{m}^{\prime}(z)$ is non-negative.

Consider a dc beam current $I$.

For a particle of charge $q$ in the beam, energy loss is $q \int W_{0}^{\prime}(z) l \frac{d z}{v} \geq 0$. 


\section{Properties of Wake Functions (cont.)}

- $\left\{\begin{aligned} v \Delta \vec{p}_{\perp} & =-q \mathcal{Q}_{m} W_{m}(z) m r^{m-1}(\hat{r} \cos m \theta-\hat{\theta} \sin m \theta) \\ v \Delta p_{s} & =-q \mathcal{Q}_{m} W_{m}^{\prime}(z) r^{m} \cos m \theta\end{aligned}\right.$

- For longitudinal, lowest azimuthal is $m=0$ or $W_{0}^{\prime}(z)$.

- For transverse, lowest azimuthal is $m=1$ or $W_{1}(z)$.

- Higher azimuthals can be important for large transverse beam size compared with pipe radius. 


\section{Properties of Wake Functions (cont.)}

- $\left\{\begin{aligned} v \Delta \vec{p}_{\perp} & =-q \mathcal{Q}_{m} W_{m}(z) m r^{m-1}(\hat{r} \cos m \theta-\hat{\theta} \sin m \theta) \\ v \Delta p_{s} & =-q \mathcal{Q}_{m} W_{m}^{\prime}(z) r^{m} \cos m \theta\end{aligned}\right.$

- For longitudinal, lowest azimuthal is $m=0$ or $W_{0}^{\prime}(z)$.

- For transverse, lowest azimuthal is $m=1$ or $W_{1}(z)$.

- Higher azimuthals can be important for large transverse beam size compared with pipe radius.

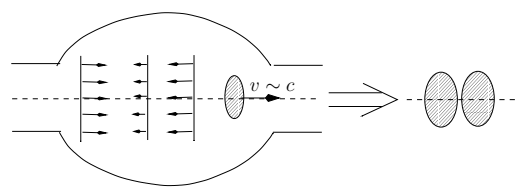

Particles in same vertical slice see same impulse. Can lead to longitudinal micro-bunching or microwave instability.

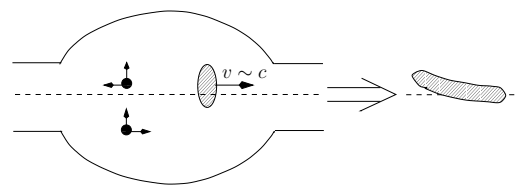

Particles in same vertical slice receive same vertical impulse independent of vertical position. Can lead to beam breakup. 


\section{Coupling Impedances}

- Beam particles form current. Component with freq. $\omega$ is $I(s, t)=\hat{l} e^{-i \omega(t-s / v)}$.

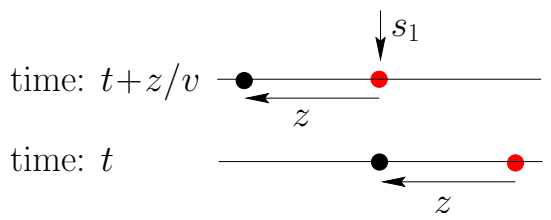

- A test particle of charge $q$ crossing a narrow discontinuity at $s_{1}$ gains energy from wake left by particles $-z$ in front $(z<0)$.

- From $v \Delta p_{s}=-q \mathcal{Q}_{m} W_{m}^{\prime}(z) r^{m} \cos m \theta$, voltage gained is $(m=0)$

$$
\begin{aligned}
V\left(s_{1}, t\right) & =-\int_{-\infty}^{\infty}\left[W_{0}^{\prime}(z)\right]_{1}\left[\hat{l} e^{-i \omega\left[(t+z / v)-s_{1} / v\right]} \frac{d z}{v}\right] \\
& =-I\left(s_{1}, t\right) \int_{-\infty}^{\infty}\left[W_{0}^{\prime}(z)\right]_{1} e^{-i \omega z / v} \frac{d z}{v} \equiv-I\left(s_{1}, t\right)\left[Z_{0}^{\|}(\omega)\right]_{1}
\end{aligned}
$$

- Defn: $Z_{0}^{\|}(\omega)=\int_{-\infty}^{\infty} W_{0}^{\prime}(z) e^{-i \omega z / v} \frac{d z}{v} \quad$ (summing over all continuities) 


\section{Coupling Impedances}

- Beam particles form current. Component with freq. $\omega$ is $I(s, t)=\hat{l} e^{-i \omega(t-s / v)}$.

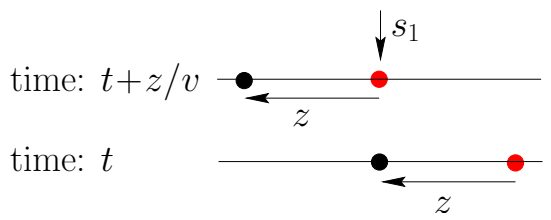

- A test particle of charge $q$ crossing a narrow discontinuity at $s_{1}$ gains energy from wake left by particles $-z$ in front $(z<0)$.

- From $v \Delta p_{s}=-q \mathcal{Q}_{m} W_{m}^{\prime}(z) r^{m} \cos m \theta$, voltage gained is $(m=0)$

$$
\begin{aligned}
V\left(s_{1}, t\right) & =-\int_{-\infty}^{\infty}\left[W_{0}^{\prime}(z)\right]_{1}\left[\hat{l} e^{-i \omega\left[(t+z / v)-s_{1} / v\right]} \frac{d z}{v}\right] \\
& =-I\left(s_{1}, t\right) \int_{-\infty}^{\infty}\left[W_{0}^{\prime}(z)\right]_{1} e^{-i \omega z / v} \frac{d z}{v} \equiv-I\left(s_{1}, t\right)\left[Z_{0}^{\|}(\omega)\right]_{1}
\end{aligned}
$$

- Defn: $Z_{0}^{\|}(\omega)=\int_{-\infty}^{\infty} W_{0}^{\prime}(z) e^{-i \omega z / v} \frac{d z}{v} \quad$ (summing over all continuities)

- Unlike a current in a circuit, a beam has transverse dimension and therefore higher multipoles.

- When the beam is off-center by amount $a$, the current $m$ th multipole is $\mathcal{Q}_{m}(s, t)=I(s, t) a^{m}=\hat{\mathcal{Q}}_{m} e^{-i \omega(t-s / v)}$. 


\section{Higher Azimuthal Impedances}

- Source particle has transverse density $\frac{\delta(r-a)}{a} \delta(\theta)$.

Subject to the $m$ th multipole element $\mathcal{Q}_{m}\left(s_{i}, t+\frac{z}{v}\right) \frac{d z}{v}$ passes location $i-z$ earlier, voltage gained by test particle is

$$
\begin{aligned}
V\left(s_{i}, t\right) & =-\int \frac{d z}{v} \mathcal{Q}_{m}\left(s_{i}, t+z / v\right)\left[W_{m}^{\prime}(z)\right]_{i} \int r d r d \theta r^{m} \cos m \theta \frac{\delta(r-a) \delta(\theta)}{a} \\
& =-\int \frac{d z}{v} \hat{\mathcal{Q}}_{m} e^{-i \omega[(t+z / v)-s / v]}\left[W_{m}^{\prime}(z)\right]_{i} a^{m} \\
& =-\frac{\mathcal{P}_{m}}{q} \mathcal{Q}_{m}\left(s_{i}, t\right) \int_{-\infty}^{0} \frac{d z}{v}\left[W_{m}^{\prime}(z)\right]_{i} e^{-i \omega z / v} \quad\left[\mathcal{P}_{m}=q a^{m}\right]
\end{aligned}
$$




\section{Higher Azimuthal Impedances}

- Source particle has transverse density $\frac{\delta(r-a)}{a} \delta(\theta)$.

Subject to the $m$ th multipole element $\mathcal{Q}_{m}\left(s_{i}, t+\frac{z}{v}\right) \frac{d z}{v}$ passes location $i-z$ earlier, voltage gained by test particle is

$$
\begin{aligned}
V\left(s_{i}, t\right) & =-\int \frac{d z}{v} \mathcal{Q}_{m}\left(s_{i}, t+z / v\right)\left[W_{m}^{\prime}(z)\right]_{i} \int r d r d \theta r^{m} \cos m \theta \frac{\delta(r-a) \delta(\theta)}{a} \\
& =-\int \frac{d z}{v} \hat{\mathcal{Q}}_{m} e^{-i \omega[(t+z / v)-s / v]}\left[W_{m}^{\prime}(z)\right]_{i} a^{m} \\
& =-\frac{\mathcal{P}_{m}}{q} \mathcal{Q}_{m}\left(s_{i}, t\right) \int_{-\infty}^{0} \frac{d z}{v}\left[W_{m}^{\prime}(z)\right]_{i} e^{-i \omega z / v} \quad\left[\mathcal{P}_{m}=q a^{m}\right]
\end{aligned}
$$

- Identify $m$ th multipole longitudinal impedance across location $i$ as

$$
\left[Z_{m}^{\|}(\omega)\right]_{i}=-\frac{q \hat{V}}{\mathcal{P}_{m} \hat{\mathcal{Q}}_{m}}=\int_{-\infty}^{\infty} \frac{d z}{v}\left[W_{m}^{\prime}(z)\right]_{i} e^{-i \omega z / v} .
$$

- Summing up around the vacuum chamber: $Z_{m}^{\|}(\omega)=\sum_{i}\left[Z_{m}^{\|}(\omega)\right]_{i}$. 


\section{Transverse Impedances}

- General defn. for long. imp.: $Z_{m}^{\|}(\omega)=\int_{-\infty}^{\infty} \frac{d z}{v} W_{m}^{\prime}(z) e^{-i \omega z / v}$.

- If we replace $W_{m}^{\prime}$ by $W_{m}$, we obtain transverse impedances

Defn. $Z_{m}^{\perp}(\omega)=\frac{i}{\beta} \int_{-\infty}^{\infty} \frac{d z}{v} W_{m}(z) e^{-i \omega z / v}$ $\left[W_{m}(z)=0\right.$ when $\left.z>0\right]$

- Long. and transverse imp. are then related by $Z_{m}^{\|}(\omega)=\frac{\omega}{c} Z_{m}^{\perp}(\omega)$, so that both $\operatorname{Re} Z_{m}^{\|}$and $\operatorname{Re} Z_{m}^{\perp}$ represent energy loss or gain.

- Transverse force, $F_{\perp} \propto-W_{m}$, must lag $\mathcal{Q}_{m}$ by $\frac{\pi}{2}$ in order for $\operatorname{Re} Z_{m}^{\perp}$ to dissipate energy. Hence the factor $i$.

- The factor $\beta$ is to cancel $\beta$ in Lorenz force, just a convention. 


\section{Direct Computation of Impedances}

- $Z_{1}^{\perp}$ can also be derived directly from the transverse force $F_{1}^{\perp}$ without going through $Z_{1}^{\|}$. 


\section{Direct Computation of Impedances}

- $Z_{1}^{\perp}$ can also be derived directly from the transverse force $F_{1}^{\perp}$ without going through $Z_{1}^{\|}$.

- When current $I(s, t)=\hat{l} e^{-i \omega(t-s / v)}$ is displaced by a transversely from axis of symmetry, deflecting force acting on a test particle is

$$
\begin{aligned}
\left\langle F_{1}^{\perp}(s, t)\right\rangle & =-\frac{q}{L} \int_{-\infty}^{\infty} W_{1}(z) a \hat{l} e^{-i \omega[(t+z / v)-s / v]} \frac{d z}{v} \\
& =-\frac{q a l(s, t)}{L} \int_{-\infty}^{\infty} W_{1}(z) e^{-i \omega z / v} \frac{d z}{v}=\frac{i \beta q l(s, t) a}{L} Z_{1}^{\perp}(\omega) .
\end{aligned}
$$

- $\langle\cdots\rangle$ implies averaged over all preceding particles. 


\section{Direct Computation of Impedances}

- $Z_{1}^{\perp}$ can also be derived directly from the transverse force $F_{1}^{\perp}$ without going through $Z_{1}^{\|}$.

- When current $I(s, t)=\hat{l} e^{-i \omega(t-s / v)}$ is displaced by a transversely from axis of symmetry, deflecting force acting on a test particle is

$$
\begin{aligned}
\left\langle F_{1}^{\perp}(s, t)\right\rangle & =-\frac{q}{L} \int_{-\infty}^{\infty} W_{1}(z) a \hat{l} e^{-i \omega[(t+z / v)-s / v]} \frac{d z}{v} \\
& =-\frac{q a l(s, t)}{L} \int_{-\infty}^{\infty} W_{1}(z) e^{-i \omega z / v} \frac{d z}{v}=\frac{i \beta q l(s, t) a}{L} Z_{1}^{\perp}(\omega) .
\end{aligned}
$$

- $\langle\cdots\rangle$ implies averaged over all preceding particles.

- For transverse: $Z_{1}^{\perp}(\omega)=-\frac{i}{q \hat{l}_{a} \beta}\left\langle\hat{F}_{1}^{\perp}\right\rangle$.

- For longitudinal: $Z_{0}^{\|}(\omega)=-\frac{1}{q \hat{l}}\left\langle\hat{F}_{0}^{\|}\right\rangle$.

- Other than from wake fcns, these are formulas employed to compute imp. directly from the long. and trans. forces seen by test particle. 
Some Properties of Impedances
(1) $Z_{m}^{\|}(\omega)=\frac{\omega}{c} Z_{m}^{\perp}(\omega)$
(P-W theorem). 


\section{Some Properties of Impedances}

(1) $Z_{m}^{\|}(\omega)=\frac{\omega}{c} Z_{m}^{\perp}(\omega) \quad(P-W$ theorem $)$.

(2) $Z_{m}^{\|}(-\omega)=\left[Z_{m}^{\|}(\omega)\right]^{*}$ and $Z_{m}^{\perp}(-\omega)=-\left[Z_{m}^{\perp}(\omega)\right]^{*} \quad\left[W_{m}(z)\right.$ is real $]$ 


\section{Some Properties of Impedances}

(1) $Z_{m}^{\|}(\omega)=\frac{\omega}{c} Z_{m}^{\perp}(\omega) \quad(P-W$ theorem $)$.

(2) $Z_{m}^{\|}(-\omega)=\left[Z_{m}^{\|}(\omega)\right]^{*}$ and $Z_{m}^{\perp}(-\omega)=-\left[Z_{m}^{\perp}(\omega)\right]^{*}$

$\left[W_{m}(z)\right.$ is real $]$

(3) $Z_{m}^{\|}(\omega)$ and $Z_{m}^{\perp}(\omega)$ are analytic, poles only in lower half $\omega$-plane.

$$
\begin{aligned}
& W_{m}(z)=-\frac{i \beta}{2 \pi} \int_{-\infty}^{\infty} Z_{m}^{\perp}(\omega) e^{i \omega z / v} d \omega \\
& W_{m}^{\prime}(z)=\frac{1}{2 \pi} \int_{-\infty}^{\infty} Z_{m}^{\|}(\omega) e^{i \omega z / v} d \omega
\end{aligned}
$$

Causality: $W_{m}(z)=W_{m}^{\prime}(z)=0$ when $z>0$.

Singularities cannot occur in upper $\omega$-plane. 


\section{Some Properties of Impedances}

(1) $Z_{m}^{\|}(\omega)=\frac{\omega}{c} Z_{m}^{\perp}(\omega) \quad(P-W$ theorem $)$.

(2) $Z_{m}^{\|}(-\omega)=\left[Z_{m}^{\|}(\omega)\right]^{*}$ and $Z_{m}^{\perp}(-\omega)=-\left[Z_{m}^{\perp}(\omega)\right]^{*}$

$\left[W_{m}(z)\right.$ is real $]$

(3) $Z_{m}^{\|}(\omega)$ and $Z_{m}^{\perp}(\omega)$ are analytic, poles only in lower half $\omega$-plane.

$W_{m}(z)=-\frac{i \beta}{2 \pi} \int_{-\infty}^{\infty} Z_{m}^{\perp}(\omega) e^{i \omega z / v} d \omega$

$W_{m}^{\prime}(z)=\frac{1}{2 \pi} \int_{-\infty}^{\infty} Z_{m}^{\|}(\omega) e^{i \omega z / v} d \omega$

$\operatorname{Re} Z_{m}^{\|}(\omega)=\frac{1}{\pi} \wp \int_{-\infty}^{\infty} d \omega^{\prime} \frac{\operatorname{Im} Z_{m}^{\|}\left(\omega^{\prime}\right)}{\omega^{\prime}-\omega}$,
Causality: $W_{m}(z)=W_{m}^{\prime}(z)=0$ when $z>0$.

Singularities cannot occur in upper $\omega$-plane. 


\section{Some Properties of Impedances}

(1) $Z_{m}^{\|}(\omega)=\frac{\omega}{c} Z_{m}^{\perp}(\omega) \quad(P-W$ theorem).

(2) $Z_{m}^{\|}(-\omega)=\left[Z_{m}^{\|}(\omega)\right]^{*}$ and $Z_{m}^{\perp}(-\omega)=-\left[Z_{m}^{\perp}(\omega)\right]^{*}$

$\left[W_{m}(z)\right.$ is real $]$

(3) $Z_{m}^{\|}(\omega)$ and $Z_{m}^{\perp}(\omega)$ are analytic, poles only in lower half $\omega$-plane.

$W_{m}(z)=-\frac{i \beta}{2 \pi} \int_{-\infty}^{\infty} Z_{m}^{\perp}(\omega) e^{i \omega z / v} d \omega$

$W_{m}^{\prime}(z)=\frac{1}{2 \pi} \int_{-\infty}^{\infty} Z_{m}^{\|}(\omega) e^{i \omega z / v} d \omega$

$\operatorname{Re} Z_{m}^{\|}(\omega)=\frac{1}{\pi} \wp \int_{-\infty}^{\infty} d \omega^{\prime} \frac{\operatorname{Im} Z_{m}^{\|}\left(\omega^{\prime}\right)}{\omega^{\prime}-\omega}, \quad \operatorname{Im} Z_{m}^{\|}(\omega)=-\frac{1}{\pi} \wp \int_{-\infty}^{\infty} d \omega^{\prime} \frac{\operatorname{Re} Z_{m}^{\|}\left(\omega^{\prime}\right)}{\omega^{\prime}-\omega}$.
Causality: $W_{m}(z)=W_{m}^{\prime}(z)=0$ when $z>0$.

Singularities cannot occur in upper $\omega$-plane.

(4) $\operatorname{Re} Z_{m}^{\|}(\omega) \geq 0$ and $\operatorname{Re} Z_{m}^{\perp}(\omega) \geq 0$ when $\omega>0$, if beam pipe has same entrance and exit cross section. (no accelerating forces) 


\section{Some Properties of Impedances}

(1) $Z_{m}^{\|}(\omega)=\frac{\omega}{c} Z_{m}^{\perp}(\omega) \quad(P-W$ theorem).

(2) $Z_{m}^{\|}(-\omega)=\left[Z_{m}^{\|}(\omega)\right]^{*}$ and $Z_{m}^{\perp}(-\omega)=-\left[Z_{m}^{\perp}(\omega)\right]^{*}$

$\left[W_{m}(z)\right.$ is real $]$

(3) $Z_{m}^{\|}(\omega)$ and $Z_{m}^{\perp}(\omega)$ are analytic, poles only in lower half $\omega$-plane.

$W_{m}(z)=-\frac{i \beta}{2 \pi} \int_{-\infty}^{\infty} Z_{m}^{\perp}(\omega) e^{i \omega z / v} d \omega$

$W_{m}^{\prime}(z)=\frac{1}{2 \pi} \int_{-\infty}^{\infty} Z_{m}^{\|}(\omega) e^{i \omega z / v} d \omega$

$\operatorname{Re} Z_{m}^{\|}(\omega)=\frac{1}{\pi} \wp \int_{-\infty}^{\infty} d \omega^{\prime} \frac{\operatorname{Im} Z_{m}^{\|}\left(\omega^{\prime}\right)}{\omega^{\prime}-\omega}, \quad \operatorname{Im} Z_{m}^{\|}(\omega)=-\frac{1}{\pi} \wp \int_{-\infty}^{\infty} d \omega^{\prime} \frac{\operatorname{Re} Z_{m}^{\|}\left(\omega^{\prime}\right)}{\omega^{\prime}-\omega}$.
Causality: $W_{m}(z)=W_{m}^{\prime}(z)=0$ when $z>0$.

Singularities cannot occur in upper $\omega$-plane.

(4) $\operatorname{Re} Z_{m}^{\|}(\omega) \geq 0$ and $\operatorname{Re} Z_{m}^{\perp}(\omega) \geq 0$ when $\omega>0$, if beam pipe has same entrance and exit cross section. (no accelerating forces)

(5) $\int_{0}^{\infty} d \omega \operatorname{Im} Z_{m}^{\perp}(\omega)=0$ and $\int_{0}^{\infty} d \omega \frac{\operatorname{Im} Z_{m}^{\|}(\omega)}{\omega}=0$. 


\section{General Behavior of Impedances}

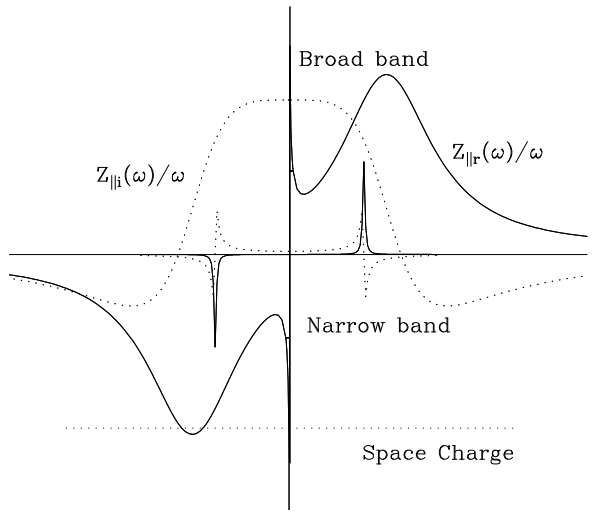

- Sharp resonances from cavities. Broad-band from bellows, BPM's, steps, etc Large values near $\omega=0$ from resistive walls.

- $-\frac{\operatorname{Im} Z_{0}^{\|}(\omega)}{\omega}$ inductive at low freq
and capacitive at high freq.

- $Z_{1}^{\perp}(\omega)$ behaves similar to $\frac{Z_{0}^{\|}(\omega)}{\omega}$ 


\section{General Behavior of Impedances}

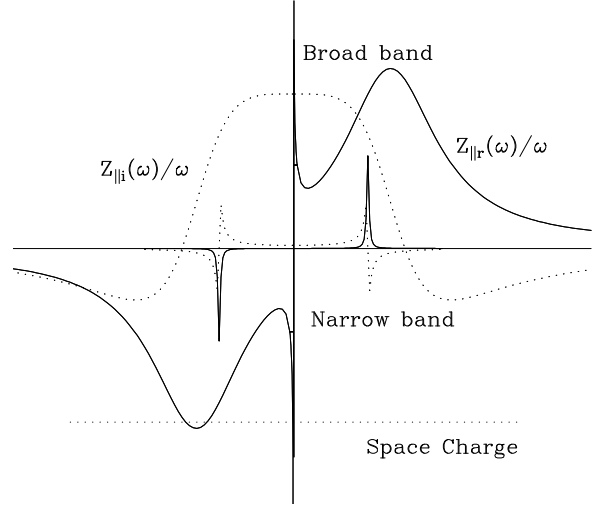

- Sharp resonances from cavities. Broad-band from bellows, BPM's, steps, etc Large values near $\omega=0$ from resistive walls.
- $-\frac{\operatorname{Im} Z_{0}^{\|}(\omega)}{\omega}$ inductive at low freq and capacitive at high freq.

- $Z_{1}^{\perp}(\omega)$ behaves similar to $\frac{Z_{0}^{\|}(\omega)}{\omega}$

- $\frac{\operatorname{Re} Z_{0}^{\|}(\omega)}{\omega}$ and $\operatorname{Re} Z_{1}^{\perp}(\omega)$ vanish at $\omega=0$.

Mathematically because of analyticity.

Physically because of no dc loss.

- At $\omega=0$, there is no Faraday's law. $\vec{E}$ and $\vec{B}$ are not related.

No image current created and no impedance. 


\section{General Comments}

- $W_{m}(z)=0, W_{m}^{\prime}(z)=0$ when $z>0$ because of causality.

- It is awkward to deal with negative $z$. Some like to use $z>0$ for particle following. Then $W_{m}(z)=0, W_{m}^{\prime}(z)=0$ when $z<0$. 


\section{General Comments}

- $W_{m}(z)=0, W_{m}^{\prime}(z)=0$ when $z>0$ because of causality.

- It is awkward to deal with negative $z$. Some like to use $z>0$ for particle following. Then $W_{m}(z)=0, W_{m}^{\prime}(z)=0$ when $z<0$.

- Then instead of

$$
Z_{m}^{\|}(\omega)=\int_{-\infty}^{\infty} e^{-i \omega z / v} W_{m}^{\prime}(z) \frac{d z}{v}, \quad W_{m}(z)=-\frac{i \beta}{2 \pi} \int_{-\infty}^{\infty} Z_{m}^{\perp}(\omega) e^{i \omega z / v} d \omega
$$

we have

$$
Z_{m}^{\|}(\omega)=\int_{-\infty}^{\infty} e^{i \omega z / v} W_{m}^{\prime}(z) \frac{d z}{v}, \quad W_{m}(z)=-\frac{i \beta}{2 \pi} \int_{-\infty}^{\infty} Z_{m}^{\perp}(\omega) e^{-i \omega z / v} d \omega
$$

and $W_{m}^{\prime}(z)=-\frac{d W_{m}(z)}{d z} . \quad \longleftarrow$ note negative sign 


\section{General Comments}

- $W_{m}(z)=0, W_{m}^{\prime}(z)=0$ when $z>0$ because of causality.

- It is awkward to deal with negative $z$. Some like to use $z>0$ for particle following. Then $W_{m}(z)=0, W_{m}^{\prime}(z)=0$ when $z<0$.

- Then instead of

$$
Z_{m}^{\|}(\omega)=\int_{-\infty}^{\infty} e^{-i \omega z / v} W_{m}^{\prime}(z) \frac{d z}{v}, \quad W_{m}(z)=-\frac{i \beta}{2 \pi} \int_{-\infty}^{\infty} Z_{m}^{\perp}(\omega) e^{i \omega z / v} d \omega
$$

we have

$$
Z_{m}^{\|}(\omega)=\int_{-\infty}^{\infty} e^{i \omega z / v} W_{m}^{\prime}(z) \frac{d z}{v}, \quad W_{m}(z)=-\frac{i \beta}{2 \pi} \int_{-\infty}^{\infty} Z_{m}^{\perp}(\omega) e^{-i \omega z / v} d \omega
$$

and $W_{m}^{\prime}(z)=-\frac{d W_{m}(z)}{d z}$. $\longleftarrow$ note negative sign

- All properties of the impedances remain unchanged, including no singularity in upper half $\omega$-plane. 


\section{General Comments}

- $W_{m}(z)=0, W_{m}^{\prime}(z)=0$ when $z>0$ because of causality.

- It is awkward to deal with negative $z$. Some like to use $z>0$ for particle following. Then $W_{m}(z)=0, W_{m}^{\prime}(z)=0$ when $z<0$.

- Then instead of

$$
Z_{m}^{\|}(\omega)=\int_{-\infty}^{\infty} e^{-i \omega z / v} W_{m}^{\prime}(z) \frac{d z}{v}, \quad W_{m}(z)=-\frac{i \beta}{2 \pi} \int_{-\infty}^{\infty} Z_{m}^{\perp}(\omega) e^{i \omega z / v} d \omega
$$

we have

$$
Z_{m}^{\|}(\omega)=\int_{-\infty}^{\infty} e^{i \omega z / v} W_{m}^{\prime}(z) \frac{d z}{v}, \quad W_{m}(z)=-\frac{i \beta}{2 \pi} \int_{-\infty}^{\infty} Z_{m}^{\perp}(\omega) e^{-i \omega z / v} d \omega
$$

and $W_{m}^{\prime}(z)=-\frac{d W_{m}(z)}{d z}$. $\longleftarrow$ note negative sign

- All properties of the impedances remain unchanged, including no singularity in upper half $\omega$-plane.

- Some may like to use $j$ instead of $i$ to denote imaginary value.

Most of the time $j=-i$. Then $Z_{m}^{\|}$and $Z_{m}^{\perp}$ have no singularity in lower half $\omega$-plane instead. 


\section{Space-Charge Impedances}

- Sp-ch imp. comes from EM fields of beam even when beam pipe is smooth and perfectly conducting.

- Want to compute $E_{s}$ due to variation

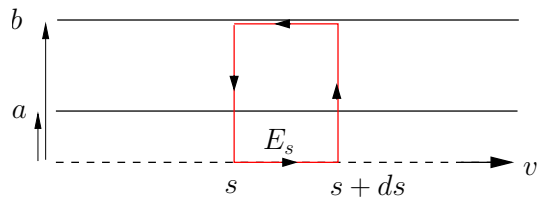
of linear density $\lambda(s-v t)$.

Assume small variation of long. dist. 


\section{Space-Charge Impedances}

- Sp-ch imp. comes from EM fields of beam even when beam pipe is smooth and perfectly conducting.

- Want to compute $E_{s}$ due to variation

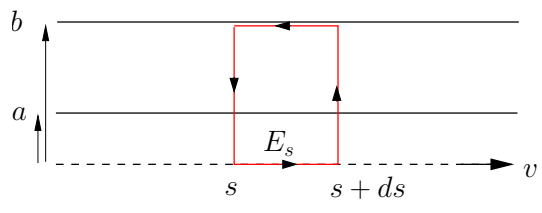
of linear density $\lambda(s-v t)$.

Assume small variation of long. dist.

- Faraday law: $\oint \vec{E} \cdot \overrightarrow{d \ell}=-\frac{\partial}{\partial t} \int \vec{B} \cdot d \vec{A}$.

$\oint \vec{E} \cdot \overrightarrow{d \ell}=E_{s} d s-\frac{e \lambda(s-v t)}{2 \pi \epsilon_{0}}\left[\int_{a}^{b} \frac{d r}{r}+\int_{0}^{a} \frac{r d r}{a^{2}}\right]+\{s \rightarrow s+d s\}$

uniform dist. assumed $\downarrow$

- Geometric factor $g_{0}=2\left[\int_{a}^{b} \frac{d r}{r}+\int_{0}^{a} \frac{r d r}{a^{2}}\right]=1+2 \ln \frac{b}{a}$. 
- Electric field or left side: $\oint \vec{E} \cdot \overrightarrow{d \ell}=E_{s} d s+\frac{e g_{0}}{4 \pi \epsilon_{0}} \frac{\partial \lambda}{\partial s} d s$.

- Magnetic field or right side:

$-\frac{\partial}{\partial t} \int \vec{B} \cdot d \vec{A}=-\frac{\partial}{\partial t} \frac{\mu_{0} e \lambda(s-v t) v}{2 \pi}\left[\int_{0}^{a} \frac{r d r}{a^{2}}+\int_{a}^{b} \frac{d r}{r}\right] d s=v^{2} \frac{e \mu_{0} g_{0}}{4 \pi} \frac{\partial \lambda}{\partial s} d s$.

- Long. field seen by particles on-axis: $E_{s}=-\frac{e g_{0}}{4 \pi \epsilon_{0} \gamma^{2}} \frac{\partial \lambda}{\partial s}$.

$c=\frac{1}{\sqrt{\epsilon_{0} \mu_{0}}}$ 
- Electric field or left side: $\oint \vec{E} \cdot \overrightarrow{d \ell}=E_{s} d s+\frac{e g_{0}}{4 \pi \epsilon_{0}} \frac{\partial \lambda}{\partial s} d s$.

- Magnetic field or right side:

$-\frac{\partial}{\partial t} \int \vec{B} \cdot d \vec{A}=-\frac{\partial}{\partial t} \frac{\mu_{0} e \lambda(s-v t) v}{2 \pi}\left[\int_{0}^{a} \frac{r d r}{a^{2}}+\int_{a}^{b} \frac{d r}{r}\right] d s=v^{2} \frac{e \mu_{0} g_{0}}{4 \pi} \frac{\partial \lambda}{\partial s} d s$.

- Long. field seen by particles on-axis: $E_{s}=-\frac{e g_{0}}{4 \pi \epsilon_{0} \gamma^{2}} \frac{\partial \lambda}{\partial s}$. $c=\frac{1}{\sqrt{\epsilon_{0} \mu_{0}}}$

- Consider a long. harmonic wave $\lambda_{1}(s ; t) \propto e^{i(n s / R-\Omega t)}$ perturbing a coasting beam of uniform linear density $\lambda_{0}$.

- Voltage drop per turn is $V=E_{s} 2 \pi R=\frac{i n e Z_{0} c g_{0}}{2 \gamma^{2}} \lambda_{1}=\frac{i n Z_{0} g_{0}}{2 \gamma^{2} \beta} I_{1}$.

- The wave constitutes a perturbing current of $I_{1}=e \lambda_{1} v$.

- Imp. is $\left.\frac{Z_{0}^{\|}}{n}\right|_{\mathrm{sp} \mathrm{ch}}=\frac{i Z_{0} g_{0}}{2 \gamma^{2} \beta}$ with $g_{0}=1+2 \ln \frac{b}{a}$.

$$
\left[Z_{0}=\sqrt{\frac{\mu_{0}}{\epsilon_{0}}}=\frac{1}{\epsilon_{0} c}=\mu_{0} c\right]
$$




\section{Comments}

- $\left.\frac{Z_{0}^{\|}}{n}\right|_{\text {sp ch }}=i \frac{Z_{0} g_{0}}{2 \beta \gamma^{2}}$ is independent of freq., but rolls off when $\omega \gtrsim \frac{\gamma c}{b}$.

- $\left.Z_{0}^{\|}\right|_{\mathrm{sp} \mathrm{ch}} \propto \omega$, resembling a neg. inductive imp. rather than a cap. imp.

- For a freq.-independent reactive imp. $\left.\frac{Z_{0}^{\|}}{n}\right|_{\mathrm{sp} \text { ch }}$, corresponding wake is $W_{0}^{\prime}(z)=-\delta^{\prime}(z)\left[i R c \beta \frac{Z_{0}^{\|}}{n}\right]_{\text {reactive }}=\delta^{\prime}(z) \frac{Z_{0} c R g_{0}}{2 \gamma^{2}}$. 


\section{Comments}

- $\left.\frac{Z_{0}^{\|}}{n}\right|_{\text {sp ch }}=i \frac{Z_{0} g_{0}}{2 \beta \gamma^{2}}$ is independent of freq., but rolls off when $\omega \gtrsim \frac{\gamma c}{b}$.

- $\left.Z_{0}^{\|}\right|_{\mathrm{sp} \mathrm{ch}} \propto \omega$, resembling a neg. inductive imp. rather than a cap. imp.

- For a freq.-independent reactive imp. $\left.\frac{Z_{0}^{\|}}{n}\right|_{\mathrm{sp} \text { ch }}$, corresponding wake is $W_{0}^{\prime}(z)=-\delta^{\prime}(z)\left[i R c \beta \frac{Z_{0}^{\|}}{n}\right]_{\text {reactive }}=\delta^{\prime}(z) \frac{Z_{0} c R g_{0}}{2 \gamma^{2}}$.

- Longitudinal reactive impedance results from a longitudinal reactive force $F_{0}^{\|}(s, t)=\left.\frac{i e^{2} v}{2 \pi} \frac{Z_{0}^{\|}}{n}\right|_{\text {reactive }} \frac{\partial \lambda(s, t)}{\partial s}$.

- This force modifies the bunch shape, called potential-well distortion. Below/above transition, capacitive force lengthens/shortens the bunch.

- Below/above transition, inductive/capacitive force can generate micro-bunching and eventual microwave instabilities. 


\section{Numerical Computation of Wakes and Impedances}

- There are numerical codes to compute wake potential of discontinuities in the vacuum chamber.

- For a cylindrical symmetric structure, there are the $\mathrm{TBCl}$ by $\mathrm{T}$. Weiland and $\mathrm{ABCl}$ by $\mathrm{Y}$. Chin, - they are called $2 \mathrm{D}$ codes. Both $m=0$ and $m=1$ modes can be computed.

- Without cylindrical symmetry, there is MAFIA by T. Weiland, with a very expensive license fee.

- The physical space is divided into grids, and the discontinuity is drawn along grid lines.

- The source cannot be a single particle.

It can be approximated by a narrow bunch, for example, Gaussian truncated with $5 \sigma$ 's.

- One need to specify the length of the wakes to be calculated. If one needs to compute impedances from the wakes by Fourier transformation, a rather long wake will be required. 
A B C I 9.2 : The Bellows of Tevatron (expanded by 10\%) $\mathrm{DDZ}=0.260 \mathrm{~mm}, \quad \mathrm{DDR}=0.320 \mathrm{~mm}$

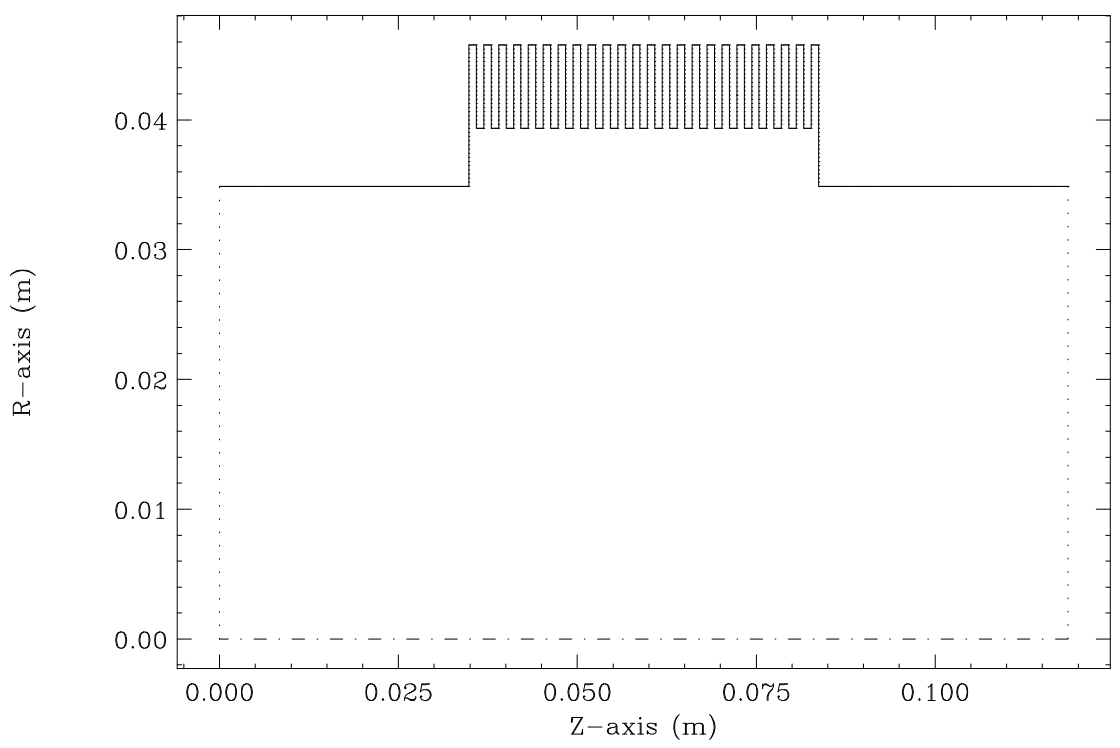

\section{K.Y. Ng (Fermilab)}


Wake Potentials

A B C I 9.2 : The Bellows of Tevatron (expanded by 10\%)

$\mathrm{MROT}=0, \quad \mathrm{SIG}=0.500 \mathrm{~cm}, \quad \mathrm{DDZ}=0.260 \mathrm{~mm}, \quad \mathrm{DDR}=0.320 \mathrm{~mm}$

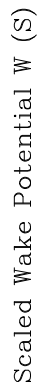

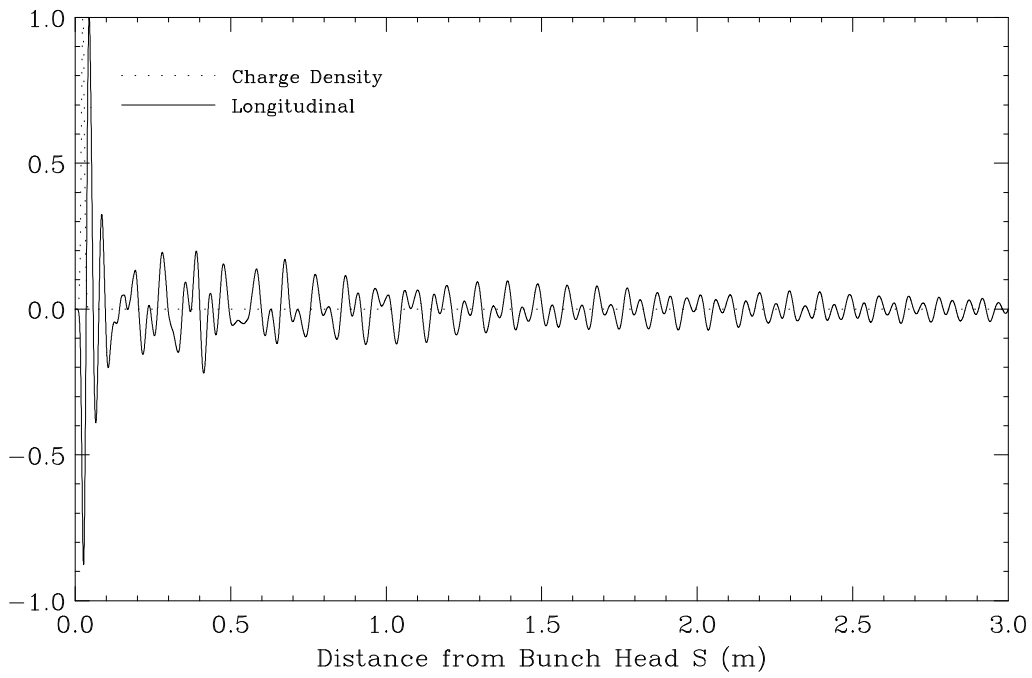

Longitudinal Wake Min $/ \mathrm{Max}=-5.711 \mathrm{E}-01 / 6.508 \mathrm{E}-01 \mathrm{~V} / \mathrm{pC}, \quad$ Loss Factor $=-3.895 \mathrm{E}-01 \mathrm{~V} / \mathrm{pC}$ 
Real Part of Longitudinal Impedance

A B C I 9.2 : The Bellows of Tevatron (expanded by 10\%)

$\mathrm{MROT}=0, \quad \mathrm{SIG}=0.500 \mathrm{~cm}, \quad \mathrm{DDZ}=0.260 \mathrm{~mm}, \quad \mathrm{DDR}=0.320 \mathrm{~mm}$

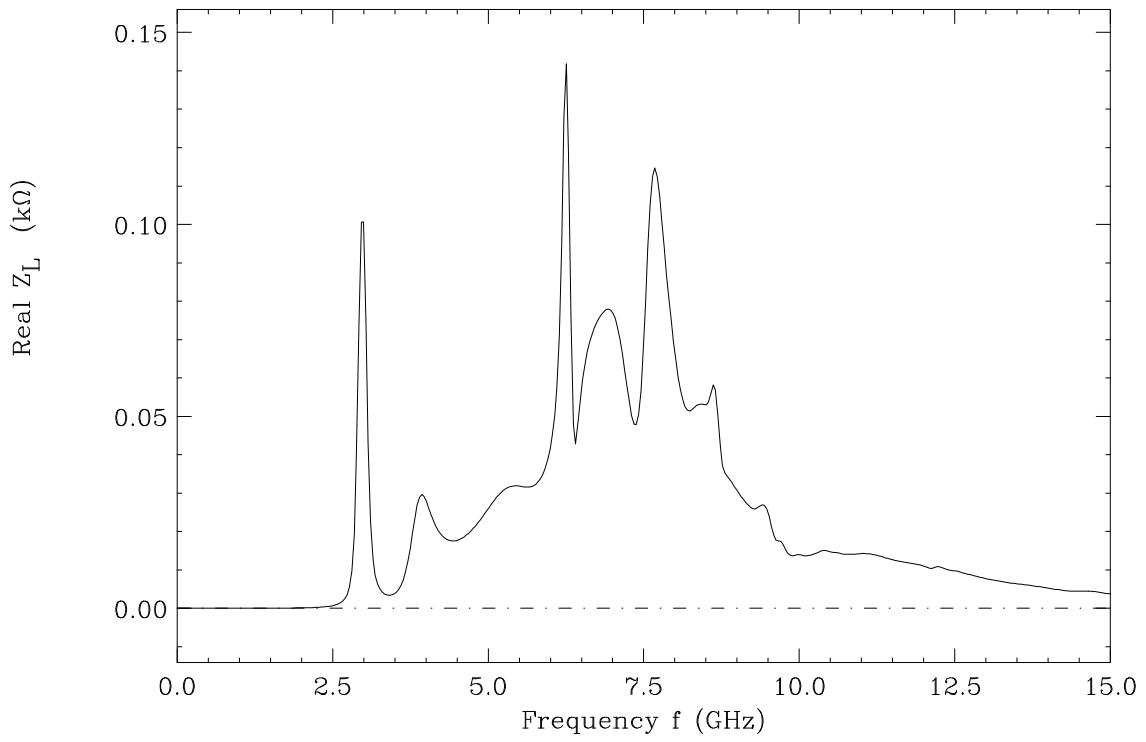


Imaginary Part of Longitudinal Impedance

A B C I 9.2 : The Bellows of Tevatron (expanded by 10\%)

$\mathrm{MROT}=0, \quad \mathrm{SIG}=0.500 \mathrm{~cm}, \quad \mathrm{DDZ}=0.260 \mathrm{~mm}, \quad \mathrm{DDR}=0.320 \mathrm{~mm}$

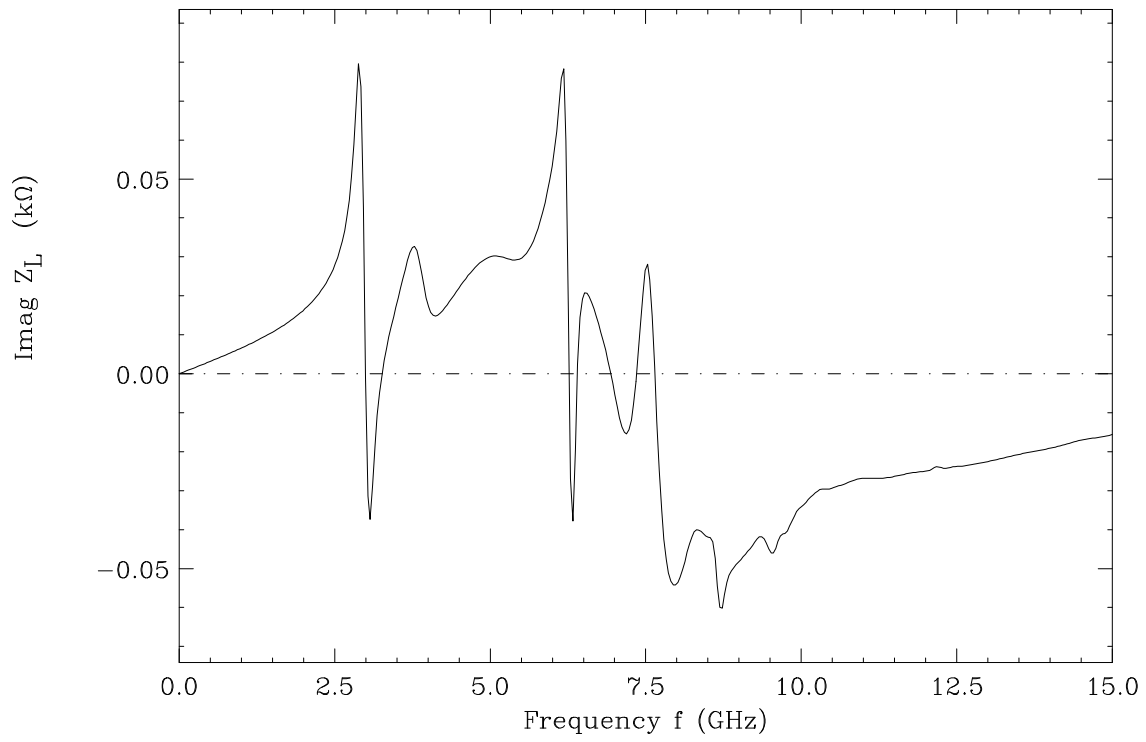


Frequency Spectrum of Loss Factor

A B C I 9.2 : The Bellows of Tevatron (expanded by 10\%)

$\mathrm{MROT}=0, \quad \mathrm{SIG}=0.500 \mathrm{~cm}, \quad \mathrm{DDZ}=0.260 \mathrm{~mm}, \quad \mathrm{DDR}=0.320 \mathrm{~mm}$

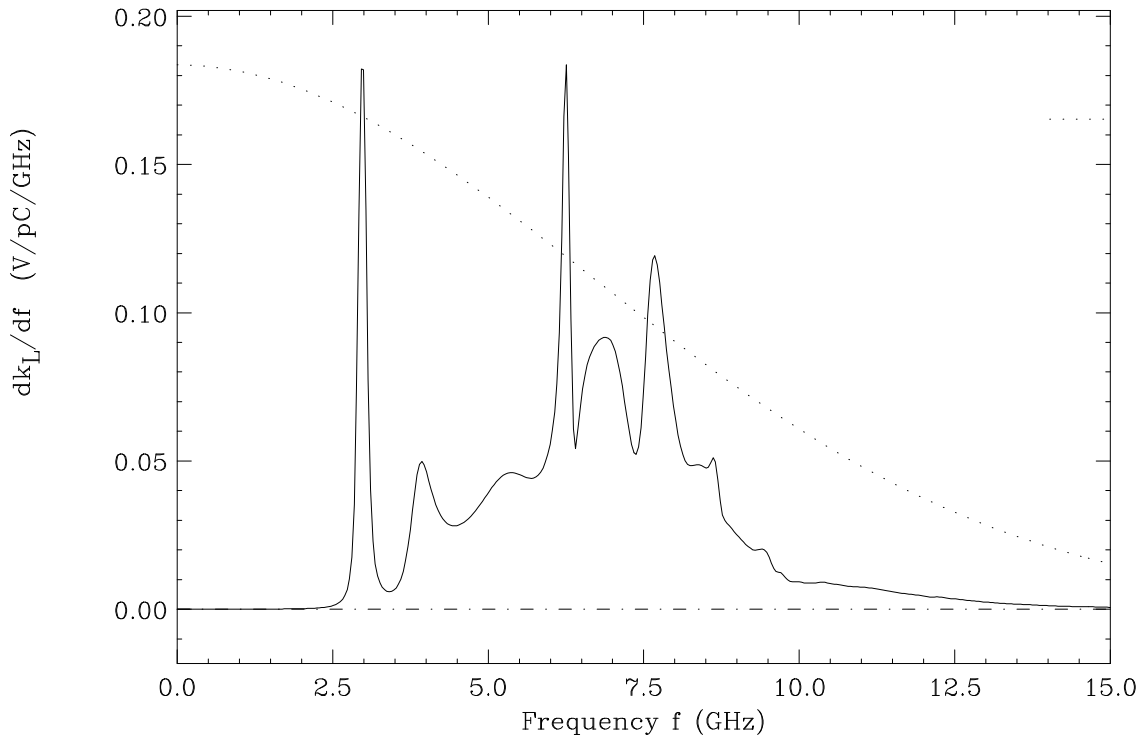


A B C I 9.2 : The Bellows of Tevatron (expanded by 10\%)

$\mathrm{MROT}=0, \quad \mathrm{SIG}=0.500 \mathrm{~cm}, \quad \mathrm{DDZ}=0.260 \mathrm{~mm}, \quad \mathrm{DDR}=0.320 \mathrm{~mm}$

$\overparen{0}$
2
$z$
$\overparen{E}$
$\stackrel{5}{1}$

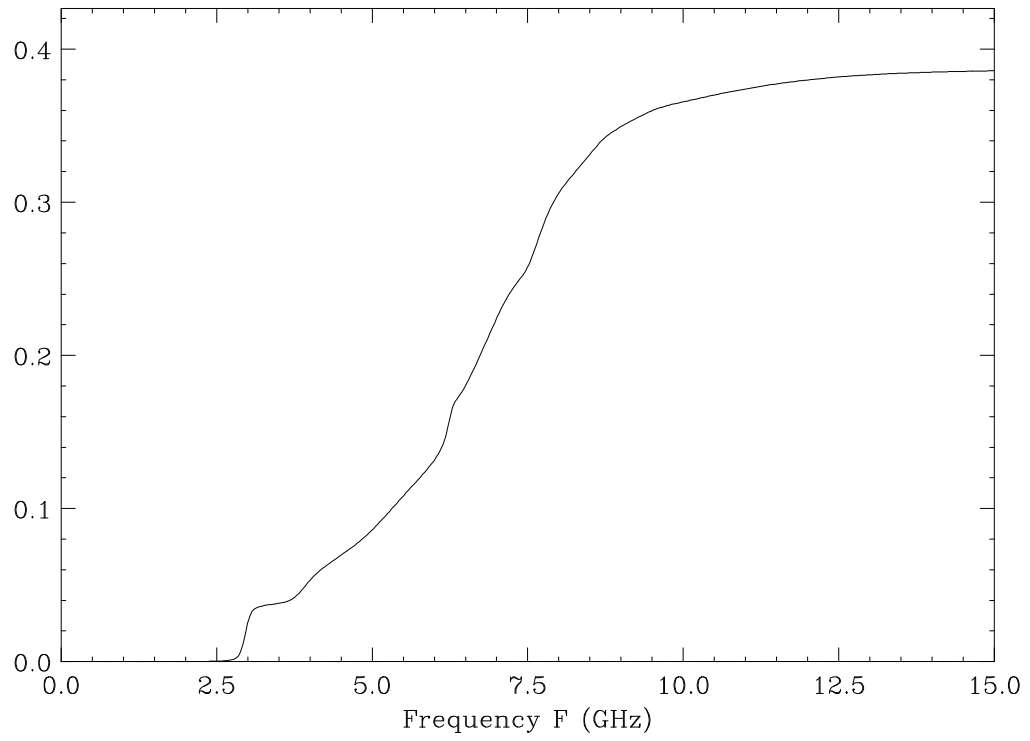




\section{Landau Damping}

- Wake force excites a number of collective waves in a beam and displaces it from its equilibrium position.

- These waves exchange energy among themselves, some grow and some are damped.

- Spread in oscillation frequency accelerates damping and decelerates growths.

- This is called Landau Damping [7, 8] 


\section{Illustration of Landau Damping [9]}

- Consider transverse oscillation of a particle in an unbunched beam $\left[\left(\frac{\partial}{\partial t}+\omega_{0} \frac{\partial}{\partial \theta}\right)^{2}+\omega_{\beta}^{2}\right] y(\theta, t)=-\frac{e l_{0}}{\gamma m C_{0}} \int_{-\infty}^{\infty} d t^{\prime} W_{1}\left(t^{\prime}-t\right)\left\langle y\left(\theta, t^{\prime}\right)\right\rangle$, with $\langle y(\theta, t)\rangle=\int \rho\left(\omega_{\beta}\right) y\left(\theta, t ; \omega_{\beta}\right) d \omega_{\beta}$

$$
\bar{\omega}=\int \omega_{\beta} \rho\left(\omega_{\beta}\right) d \omega_{\beta}
$$

- Go to frequency domain by introducing

$$
\tilde{y}_{n}(\Omega)=\frac{1}{4 \pi^{2}} \int_{-\infty}^{\infty} d t \int d \theta y(\theta, t) e^{-i n \theta+i \Omega t}
$$

- Here, we solve as an initial-value problem. For $t<0, y(\theta, t)=0$.

Beam receives a kick at $t=0$, or $\frac{\partial y(\theta, 0)}{\partial t}=\sum_{n} \dot{y}_{n 0} e^{i n \theta}$.

- R.S. $=\frac{i e c l_{0} Z_{1}^{\perp}(\Omega)}{E_{0} T_{0}}\left\langle\tilde{y}_{n}\right\rangle \equiv-2 \bar{\omega}(\Delta \omega)_{0}\left\langle\tilde{y}_{n}\right\rangle$

$$
(\Delta \omega)_{0} \text { is }\left\{\begin{array}{l}
\text { freq shift } w / o \\
\text { Landau damping }
\end{array}\right.
$$

- L.S. $=\left(\omega_{\beta}^{2}-\hat{\omega}^{2}\right) \tilde{y}_{n}(\Omega)+\left.\frac{1}{4 \pi^{2}} \int d \theta\left[\frac{\partial y}{\partial t}-i\left(\Omega-2 n \omega_{0}\right) y\right] e^{-i n \theta+i \Omega t}\right|_{0} ^{\infty}$

$$
=\left(\omega_{\beta}^{2}-\hat{\omega}^{2}\right) \tilde{y}_{n}(\Omega)-\frac{\dot{y}_{n 0}}{2 \pi} \leftarrow \text { for } \operatorname{Im} \Omega>0 \text { only. }
$$

$$
\hat{\omega}=\Omega-n \omega_{0}
$$


- Soln. for $\operatorname{Im} \Omega>0$ (upper $\Omega$-plane): $\tilde{y}_{n}(\Omega)=-\frac{2 \bar{\omega}(\Delta \omega)_{0}}{\omega_{\beta}^{2}-\hat{\omega}^{2}}\left\langle\tilde{y}_{n}\right\rangle+\frac{\dot{y}_{n 0}}{2 \pi\left(\omega_{\beta}^{2}-\hat{\omega}^{2}\right)}$

- Now average over the whole beam by $\int d \omega_{\beta} \rho\left(\omega_{\beta}\right) \times \ldots$

- $\left[1+2 \bar{\omega}(\Delta \omega)_{0} \int d \omega_{\beta} \frac{\rho\left(\omega_{\beta}\right)}{\omega_{\beta}^{2}-\hat{\omega}^{2}}\right]\left\langle\tilde{y}_{n}\right\rangle=\frac{\dot{y}_{n 0}}{2 \pi} \int d \omega_{\beta} \frac{\rho\left(\omega_{\beta}\right)}{\omega_{\beta}^{2}-\hat{\omega}^{2}}$

$\uparrow H(\Omega)$

- Thus $\left\langle\tilde{y}_{n}(\Omega)\right\rangle=\frac{\frac{\dot{y}_{n 0}}{2 \pi} \int d \omega_{\beta} \frac{\rho\left(\omega_{\beta}\right)}{\omega_{\beta}^{2}-\hat{\omega}^{2}}}{H(\Omega)}$ $\hat{\omega}=\Omega-n \omega_{0}$

- The inverse Fourier transform $\langle y(\theta, t)\rangle=\sum_{n} \int d \Omega\left\langle\tilde{y}_{n}(\Omega)\right\rangle e^{-i(\Omega t-n \theta)}$ gives solution in $\theta-t$ space.

- However, need to know $\left\langle\tilde{y}_{n}(\Omega)\right\rangle$ over the whole $\Omega$-plane.

- Notice that in above $\left\langle\tilde{y}_{n}(\Omega)\right\rangle$ is discontinuous across $\operatorname{Im} \Omega=0$

- $\hat{\omega}=\hat{\omega}_{R} \pm i \epsilon \longrightarrow \int \frac{\rho\left(\omega_{\beta}\right) d \omega_{\beta}}{\omega_{\beta}-\hat{\omega}}=\int \frac{\rho\left(\omega_{\beta}\right) d \omega_{\beta}}{\omega_{\beta}-\hat{\omega}_{R} \mp i \epsilon}=\wp \int d \omega_{\beta} \frac{\rho\left(\omega_{\beta}\right)}{\omega_{\beta}-\hat{\omega}_{R}} \pm i \pi \rho\left(\hat{\omega}_{R}\right)$ 


\section{Analytic Continuation}

- Reason of discontinuity is path of integration.

- Top: $\operatorname{Im} \Omega>0$

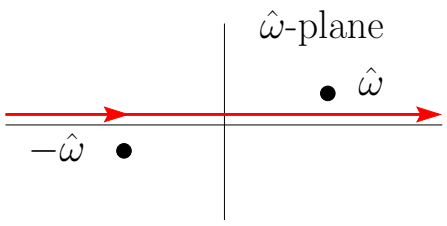

We know how the contour goes.

- Middle: $\operatorname{Im} \Omega<0$ and $\left\langle\tilde{y}_{n}(\Omega)\right\rangle$ not defined.

If same contour is followed, result will be different.

Discontinuity occurs across $\operatorname{Im} \Omega=0$.

- Bottom: If we follow Landau contour C, there will not be any discontinuity.
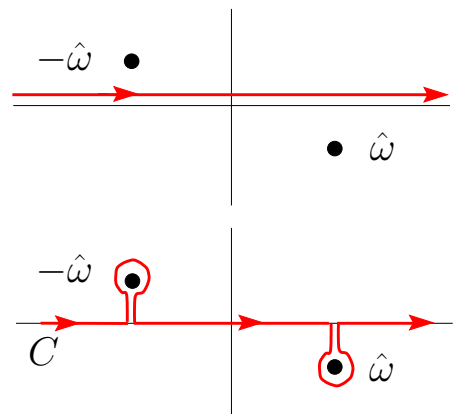

- Contour $C$ is always below pole at $\hat{\omega}$ and above pole at $-\hat{\omega}$.

This is what we mean by analytic continuation. 
- $\langle y(\theta, t)\rangle=\sum_{n} \int_{W} d \Omega \frac{\dot{y}_{n 0} \int_{C} d \omega_{\beta} \frac{\rho\left(\omega_{\beta}\right)}{\omega_{\beta}^{2}-\hat{\omega}^{2}}}{2 \pi H(\Omega)} e^{-i \Omega t+i n \theta}$

where $W$ is path above all singularities so that $\langle y(\theta, t)\rangle=0$ at $t<0$.

- $\langle y(\theta, t)\rangle=\sum_{k} \operatorname{Res}\left[\sum_{n} \frac{-i \dot{y}_{n 0} \int_{C} d \omega_{\beta} \frac{\rho\left(\omega_{\beta}\right)}{\omega_{\beta}^{2}-\hat{\omega}^{2}}}{H(\Omega)}\right]_{\Omega=\Omega_{k}} e^{-i \Omega_{k} t+i n \theta}$,

where $\Omega_{k}$ is $k^{\text {th }}$ zero of $H(\Omega)$.

(1) The poles of $H(\Omega)$ gives all waves excited

(2) A pole with $\operatorname{Im} \Omega_{k}>0$ implies growing

(3) A pole with $\operatorname{Im} \Omega_{k}<0$ implies damping

(4) $\therefore$ it is essential to solve $H(\Omega)=0$, which is known as the dispersion relation. 


\section{Dispersion Relation}

- $H(\Omega)=1+2 \bar{\omega}(\Delta \omega)_{0} \int_{C} d \omega_{\beta} \frac{\rho\left(\omega_{\beta}\right)}{\omega_{\beta}^{2}-\hat{\omega}^{2}}=0 \quad$ with $\hat{\omega}=\Omega-n \omega_{0}$

- Since $\pm \hat{\omega}$ are far apart, can linearize to get

$$
1+(\Delta \omega)_{0} \int_{C} d \omega_{\beta} \frac{\rho\left(\omega_{\beta}\right)}{\omega_{\beta}-\Omega+n \omega_{0}}=0
$$

- It gives excitation freq. $\Omega$ as a fcn. of wave number $n$, hence called dispersion relation.

- Can also write $1+(\Delta \omega)_{0} \int_{C} d \omega_{\beta} \frac{\rho\left(\omega_{\beta}\right)}{\left(\omega_{\beta}-\bar{\omega}\right)-\left(\Omega-n \omega_{0}-\bar{\omega}\right)}=0$

- When spread in $\omega_{\beta}$ is small, $\omega_{\beta}-\bar{\omega} \rightarrow 0$, get $(\Delta \omega)_{0}=\Omega-n \omega_{0}-\bar{\omega}$, which is just betatron freq. shift in the absence of Landau damping.

- Note that this is not a coherent shift.

It is the shift driven by the wake or imp. — called dynamic shift actually dynamic shift $=$ coherent shift - incoherent shift 


\section{Coherent, Incoherent, and Impedance Forces}

- Vertical force on a beam particle $\frac{d^{2} y}{d s^{2}}+\frac{\nu_{0 y}^{2}}{R^{2}} y=\frac{F(y, \bar{y})}{\gamma m v^{2}}$. 


\section{Coherent, Incoherent, and Impedance Forces}

- Vertical force on a beam particle $\frac{d^{2} y}{d s^{2}}+\frac{\nu_{0 y}^{2}}{R^{2}} y=\frac{F(y, \bar{y})}{\gamma m v^{2}}$.

- For small offsets, $\frac{d^{2} y}{d s^{2}}+\frac{\nu_{0 y}^{2}}{R^{2}} y=\frac{1}{\gamma m v^{2}}\left(\left.\frac{\partial F}{\partial y}\right|_{\bar{y}=0} y+\left.\frac{\partial F}{\partial \bar{y}}\right|_{y=0} \bar{y}\right)$. 


\section{Coherent, Incoherent, and Impedance Forces}

- Vertical force on a beam particle $\frac{d^{2} y}{d s^{2}}+\frac{\nu_{0 y}^{2}}{R^{2}} y=\frac{F(y, \bar{y})}{\gamma m v^{2}}$.

- For small offsets, $\frac{d^{2} y}{d s^{2}}+\frac{\nu_{0 y}^{2}}{R^{2}} y=\frac{1}{\gamma m v^{2}}\left(\left.\frac{\partial F}{\partial y}\right|_{\bar{y}=0} y+\left.\frac{\partial F}{\partial \bar{y}}\right|_{y=0} \bar{y}\right)$.

- For center of mass, $\frac{d^{2} \bar{y}}{d s^{2}}+\frac{\nu_{0 y}^{2}}{R^{2}} \bar{y}=\frac{1}{\gamma m v^{2}}\left(\left.\frac{\partial F}{\partial y}\right|_{\bar{y}=0}+\left.\frac{\partial F}{\partial \bar{y}}\right|_{y=0}\right) \bar{y}$. 


\section{Coherent, Incoherent, and Impedance Forces}

- Vertical force on a beam particle $\frac{d^{2} y}{d s^{2}}+\frac{\nu_{0 y}^{2}}{R^{2}} y=\frac{F(y, \bar{y})}{\gamma m v^{2}}$.

- For small offsets, $\frac{d^{2} y}{d s^{2}}+\frac{\nu_{0 y}^{2}}{R^{2}} y=\frac{1}{\gamma m v^{2}}\left(\left.\frac{\partial F}{\partial y}\right|_{\bar{y}=0} y+\left.\frac{\partial F}{\partial \bar{y}}\right|_{y=0} \bar{y}\right)$.

- For center of mass, $\frac{d^{2} \bar{y}}{d s^{2}}+\frac{\nu_{0 y}^{2}}{R^{2}} \bar{y}=\frac{1}{\gamma m v^{2}}\left(\left.\frac{\partial F}{\partial y}\right|_{\bar{y}=0}+\left.\frac{\partial F}{\partial \bar{y}}\right|_{y=0}\right) \bar{y}$.

- Thus $\left.\Delta \nu_{y \text { inc }} \propto \frac{\partial F}{\partial y}\right|_{\bar{y}=0}$

$$
\left.\Delta \nu_{y \operatorname{coh}} \propto \frac{\partial F}{\partial y}\right|_{\bar{y}=0}+\left.\frac{\partial F}{\partial \bar{y}}\right|_{y=0}
$$




\section{Coherent, Incoherent, and Impedance Forces}

- Vertical force on a beam particle $\frac{d^{2} y}{d s^{2}}+\frac{\nu_{0 y}^{2}}{R^{2}} y=\frac{F(y, \bar{y})}{\gamma m v^{2}}$.

- For small offsets, $\frac{d^{2} y}{d s^{2}}+\frac{\nu_{0 y}^{2}}{R^{2}} y=\frac{1}{\gamma m v^{2}}\left(\left.\frac{\partial F}{\partial y}\right|_{\bar{y}=0} y+\left.\frac{\partial F}{\partial \bar{y}}\right|_{y=0} \bar{y}\right)$.

- For center of mass, $\frac{d^{2} \bar{y}}{d s^{2}}+\frac{\nu_{0 y}^{2}}{R^{2}} \bar{y}=\frac{1}{\gamma m v^{2}}\left(\left.\frac{\partial F}{\partial y}\right|_{\bar{y}=0}+\left.\frac{\partial F}{\partial \bar{y}}\right|_{y=0}\right) \bar{y}$.

- Thus $\left.\Delta \nu_{y \text { inc }} \propto \frac{\partial F}{\partial y}\right|_{\bar{y}=0}$

$$
\left.\Delta \nu_{y \operatorname{coh}} \propto \frac{\partial F}{\partial y}\right|_{\bar{y}=0}+\left.\frac{\partial F}{\partial \bar{y}}\right|_{y=0}
$$

- But $\left.Z_{1}^{\perp} \propto \frac{\partial F}{\partial \bar{y}}\right|_{y=0}$,

- $\therefore$ Impedance Shift $=$ Coherent Shift - Incoherent Shift.

$\uparrow$ also called dynamic shift 
- $\Delta \nu_{y \text { coh }}$ : result of all forces acting on center of beam at $\bar{y}$.

- $Z_{1}^{\perp}$ : force generated by center motion of beam on individual particle. 
- $\Delta \nu_{y \text { coh }}$ : result of all forces acting on center of beam at $\bar{y}$.

- $Z_{1}^{\perp}$ : force generated by center motion of beam on individual particle.

- Example: a beam between two infinite horizontal conducting planes.

- Horizontal translational invariance $\Longrightarrow$ horizontal image force acting at center of beam vanishes independent of whether beam is oscillating horizontally or vertically. $\therefore \Delta \nu_{x \text { coh }}=0$, but $Z_{1}^{H} \neq 0$. 
- $\Delta \nu_{y \text { coh }}$ : result of all forces acting on center of beam at $\bar{y}$.

- $Z_{1}^{\perp}$ : force generated by center motion of beam on individual particle.

- Example: a beam between two infinite horizontal conducting planes.

- Horizontal translational invariance $\Longrightarrow$ horizontal image force acting at center of beam vanishes independent of whether beam is oscillating horizontally or vertically. $\therefore \Delta \nu_{x \text { coh }}=0$, but $Z_{1}^{H} \neq 0$.

- Single bunch tune shift measurement at CERN SPS. [5]
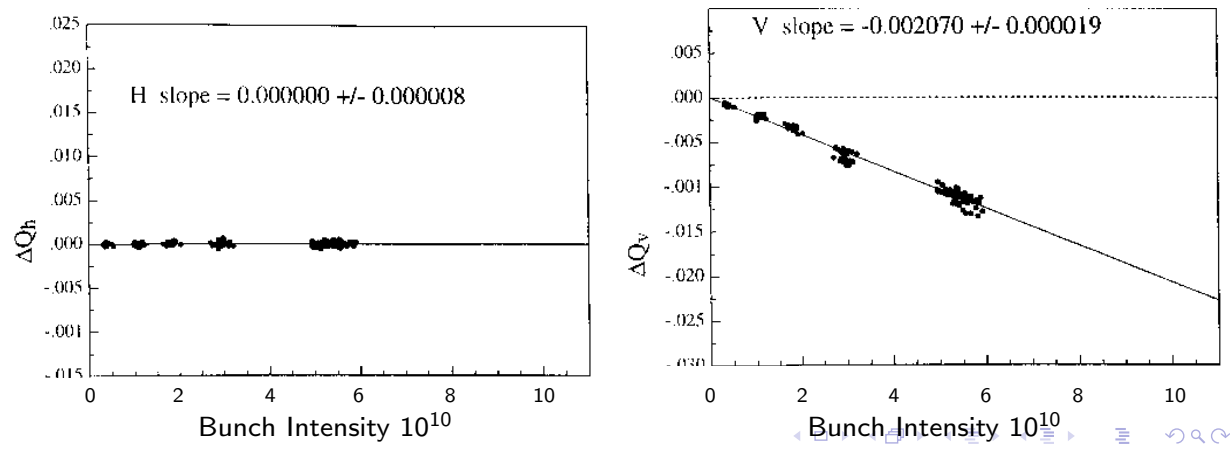


\section{Decoherence vs Landau Damping}

- Start with a displacement.

Because particles oscillate with slightly different frequencies, very soon oscillations will not be in phase, and average displacement decays to zero.

- For decoherence, we do not need any force.

- This problem is easy to solve.

However, let's go back to our derivation, and see what happen when the force is zero.

$\begin{aligned}\langle y(\theta, t)\rangle & =\sum_{n} \int_{W} d \Omega \frac{\dot{y}_{n 0} \int_{C} d \omega_{\beta} \frac{\rho\left(\omega_{\beta}\right)}{\omega_{\beta}^{2}-\hat{\omega}^{2}}}{2 \pi H(\Omega)} e^{-i \Omega t+i n \theta} \\ \langle y(\theta, t)\rangle & =\sum_{n} \frac{\dot{y}_{n 0}}{2 \pi} e^{i n\left(\theta-\omega_{0} t\right)} \int_{C} d \omega_{\beta} \int_{W} d \hat{\omega} \frac{\rho\left(\omega_{\beta}\right)}{\omega_{\beta}^{2}-\hat{\omega}^{2}} e^{-i \hat{\omega} t}\end{aligned}$

- We integrate over $\hat{\omega}$ by picking up the residue of two poles. 
- For $t \geq 0,\langle y(\theta, t)\rangle=\sum_{n} \frac{\dot{y}_{n 0}}{2 \pi} e^{i n\left(\theta-\omega_{0} t\right)} \int d \omega_{\beta} \frac{\rho\left(\omega_{\beta}\right)}{\omega_{\beta}} \sin \omega_{\beta} t$

- We see that at the impact of a velocity, the average displacement increases from zero to a maximum, then decays $\longrightarrow$ decoheres.

- Want to check result at $t=0$. For $t \geq 0$,

$$
\begin{aligned}
\langle\dot{y}(\theta, t)\rangle= & \sum_{n} \frac{\dot{y}_{n 0}}{2 \pi} e^{i n\left(\theta-\omega_{0} t\right)} \int d \omega_{\beta} \rho\left(\omega_{\beta}\right) \cos \omega_{\beta} t \\
& -\sum_{n} \frac{i n \omega_{0} \dot{y}_{n 0}}{2 \pi} e^{i n\left(\theta-\omega_{0} t\right)} \int d \omega_{\beta} \frac{\rho\left(\omega_{\beta}\right)}{\omega_{\beta}} \sin \omega_{\beta} t .
\end{aligned}
$$

- Thus $\langle\dot{y}(\theta, t)\rangle=\sum_{n} \frac{\dot{y}_{n 0}}{2 \pi} e^{i n \theta}=\dot{y}(\theta, 0)$ as required.

- Notice that Landau damping requires a dynamic force. Here, it is the wake force or impedance.

- So decoherence is kinematic, while Landau damping is dynamic. 


\section{A Solvable Example}

- Consider the Lorentzian distribution $\rho\left(\omega_{\beta}\right)=\frac{S_{\frac{1}{2}}}{\pi} \frac{1}{\left(\omega_{\beta}-\hat{\omega}\right)^{2}+S_{\frac{1}{2}}^{2}}$

- $\int_{C} \frac{\rho\left(\omega_{\beta}\right) d \omega_{\beta}}{\omega_{\beta}^{2}-\hat{\omega}^{2}}=\frac{S_{\frac{1}{2}}}{\pi} \int_{C} \frac{d \omega_{\beta}}{\left(\omega_{\beta}-\hat{\omega}\right)\left(\omega_{\beta}+\hat{\omega}\right)\left(\omega_{\beta}-\bar{\omega}+i S_{\frac{1}{2}}\right)\left(\omega_{\beta}-\bar{\omega}-i S_{\frac{1}{2}}\right)}$

- Can choose closing contour at top or bottom of $\omega_{\beta}$-plane.

- Pick up only 2 poles.

- The moral of this example is easy integration.

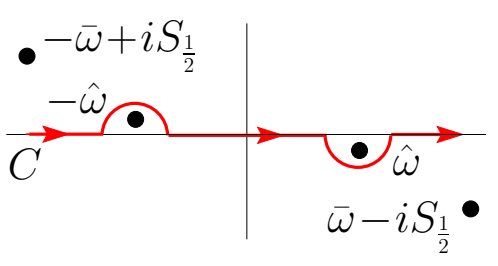

- $\int_{C} \frac{\rho\left(\omega_{\beta}\right) d \omega_{\beta}}{\omega_{\beta}^{2}-\bar{\omega}^{2}}=-\frac{\hat{\omega}+i S_{\frac{1}{2}}}{\hat{\omega}\left(\hat{\omega}-\bar{\omega}+i S_{\frac{1}{2}}\right)\left(\hat{\omega}+\bar{\omega}+i S_{\frac{1}{2}}\right)}$

- $H(\Omega)=1+2 \bar{\omega}(\Delta \omega)_{0} \int_{C} \frac{\rho\left(\omega_{\beta}\right) d \omega_{\beta}}{\omega_{\beta}^{2}-\bar{\omega}^{2}}$

$$
=\frac{\hat{\omega}\left(\hat{\omega}-\bar{\omega}+i S_{\frac{1}{2}}\right)\left(\hat{\omega}+\bar{\omega}+i S_{\frac{1}{2}}\right)-2 \bar{\omega}(\Delta \omega)_{0}\left(\hat{\omega}+i S_{\frac{1}{2}}\right)}{\hat{\omega}\left(\hat{\omega}-\bar{\omega}+i S_{\frac{1}{2}}\right)\left(\hat{\omega}+\bar{\omega}+i S_{\frac{1}{2}}\right)}
$$


- $\langle y(\theta, t)\rangle=\sum_{n} \dot{y}_{n 0} \int_{W} \frac{d \hat{\omega}}{2 \pi} \frac{\left(\hat{\omega}+i S_{\frac{1}{2}}\right) e^{-i\left(\hat{\omega}+n \omega_{0}\right) t+i n \theta}}{\hat{\omega}\left(\hat{\omega}-\bar{\omega}+i S_{\frac{1}{2}}\right)\left(\hat{\omega}+\bar{\omega}+i S_{\frac{1}{2}}\right)-2 \bar{\omega}(\Delta \omega)_{0}\left(\hat{\omega}+i S_{\frac{1}{2}}\right)}$

- Approximation: freq. shift $(\Delta \omega)_{0}$ and freq. spread $S_{\frac{1}{2}}$ are small $\frac{\left|(\Delta \omega)_{0}\right|}{\bar{\omega}} \ll 1$ and $\frac{S_{\frac{1}{2}}}{\bar{\omega}} \ll 1$

- denom $=\hat{\omega}\left[\hat{\omega}-\bar{\omega}-(\Delta \omega)_{0}+i S_{\frac{1}{2}}\right]\left[\hat{\omega}+\bar{\omega}+(\Delta \omega)_{0}+i S_{\frac{1}{2}}\right]+\left[-2 i \bar{\omega}(\Delta \omega)_{0} S_{\frac{1}{2}}+\hat{\omega}(\Delta \omega)_{0}^{2}\right]$

$$
\approx \hat{\omega}\left[\hat{\omega}-\bar{\omega}-(\Delta \omega)_{0}+i S_{\frac{1}{2}}\right]\left[\hat{\omega}+\bar{\omega}+(\Delta \omega)_{0}+i S_{\frac{1}{2}}\right]
$$

- 3 solutions:

$$
\Omega=\left\{\begin{array}{l}
n \omega_{0} \\
n \omega_{0}+\bar{\omega}+(\Delta \omega)_{0}-i S_{\frac{1}{2}} \\
n \omega_{0}-\bar{\omega}-(\Delta \omega)_{0}-i S_{\frac{1}{2}}
\end{array}=\left\{\begin{array}{l}
n \omega_{0} \\
n \omega_{0}+\bar{\omega}+(\Delta \omega)_{0 R}-i\left(S_{\frac{1}{2}}-(\Delta \omega)_{01}\right) \\
n \omega_{0}-\bar{\omega}-(\Delta \omega)_{0 R}-i\left(S_{\frac{1}{2}}+(\Delta \omega)_{0}\right)
\end{array}\right.\right.
$$

- 1st soln. consists of higher-harmonic revolution waves without freq. perturbation and is of not much interest.

- 2nd and 3rd are the same physically. We will show their combined result. 
- $\langle y(\theta, t)\rangle=\sum_{n} \frac{-i \dot{y}_{n 0}}{2 \bar{\omega}} e^{i n \theta-i\left[n \omega_{0}+\bar{\omega}+(\Delta \omega)_{0 R}\right] t} e^{-\left[S_{\frac{1}{2}}-(\Delta \omega)_{01}\right] t}$ with $(\Delta \omega)_{0_{R}}=\frac{e c l_{0} \mathcal{I} m Z_{1}^{\perp}(\Omega)}{2 \bar{\omega} E_{0} T_{0}},(\Delta \omega)_{0 \prime}=-\frac{e c l_{0} \operatorname{Re} Z_{1}^{\perp}(\Omega)}{2 \bar{\omega} E_{0} T_{0}}, \Omega=n \omega_{0}+(\Delta \omega)_{0}$

- Distr. spread $S_{\frac{1}{2}}$ always provides damping, but $(\Delta \omega)_{0} \gtrless 0$ grows/damps.

- Since $\operatorname{Re} Z_{1}^{\perp}(\Omega) \gtrless 0$ for $\Omega \gtrless 0$, damping/growth is related to $\Omega \gtrless 0$ or fast/slow waves.

- If $S_{\frac{1}{2}} \gtrless\left|(\Delta \omega)_{0}\right|$, slow waves damp/grow.

- This mechanism is called Landau Damping. 
- $\langle y(\theta, t)\rangle=\sum_{n} \frac{-i \dot{y}_{n 0}}{2 \bar{\omega}} e^{i n \theta-i\left[n \omega_{0}+\bar{\omega}+(\Delta \omega)_{0 R}\right] t} e^{-\left[S_{\frac{1}{2}}-(\Delta \omega)_{01}\right] t}$ with $(\Delta \omega)_{0_{R}}=\frac{e c l_{0} \mathcal{I} m Z_{1}^{\perp}(\Omega)}{2 \bar{\omega} E_{0} T_{0}},(\Delta \omega)_{0 \prime}=-\frac{e c l_{0} \operatorname{Re} Z_{1}^{\perp}(\Omega)}{2 \bar{\omega} E_{0} T_{0}}, \Omega=n \omega_{0}+(\Delta \omega)_{0}$

- Distr. spread $S_{\frac{1}{2}}$ always provides damping, but $(\Delta \omega)_{0,} \gtrless 0$ grows/damps.

- Since $\operatorname{Re} Z_{1}^{\perp}(\Omega) \gtrless 0$ for $\Omega \gtrless 0$, damping/growth is related to $\Omega \gtrless 0$ or fast/slow waves.

- If $S_{\frac{1}{2}} \gtrless\left|(\Delta \omega)_{0}\right|$, slow waves damp/grow.

- This mechanism is called Landau Damping.

- Note that even $\langle y(\theta, t)\rangle$ is damped to zero, displacements of individual particles are not. $\longleftarrow$ important

- In practice, any small initial ripples of the beam center will be damped if $S_{\frac{1}{2}}>\left|(\Delta \omega)_{0, l}\right|$, so that $\operatorname{Re} Z_{1}^{\perp}$ cannot drive individual displacements.

- In other words, Landau damping nips any instability growth in the bud. 


\section{Stability Region Plot $[10]$}

- Spread in particle distribution provides Landau damping.

- For a given particle distribution $\rho\left(\omega_{\beta}\right)$, wish to know what $Z_{1}^{\perp}$ will ensure beam stability - the so-called stability contour.

- For this, need to solve the dispersion relation

$$
1+(\Delta \omega)_{0} \int_{C} d \omega_{\beta} \frac{\rho\left(\omega_{\beta}\right)}{\omega_{\beta}-\Omega+n \omega_{0}}=0
$$$$
\hat{\omega}=\Omega-n \omega_{0}
$$

- We believe the HWHM provides a better description of distribution than half width or rms width.

Want to express all variables in terms of $S_{\frac{1}{2}}$.

- Let $u=\frac{\hat{\omega}-\bar{\omega}}{S_{\frac{1}{2}}}, \quad v=\frac{\omega_{\beta}-\bar{\omega}}{S_{\frac{1}{2}}}, \quad \hat{\rho}(v)=S_{\frac{1}{2}} \rho\left(\omega_{\beta}\right)$ so that $\int \hat{\rho}(v) d v=1$

- We are interested in the stability contour or the threshold of instability. So let $i \operatorname{Im} \hat{\omega} \longrightarrow+i \epsilon$ with $\epsilon=0^{+}$

- The same as letting $u \longrightarrow u+i \epsilon$ with $u$ real. 
- Dispersion relation becomes $1+\frac{(\Delta \omega)_{0}}{S_{\frac{1}{2}}} \int_{-\infty}^{\infty} \frac{\hat{\rho}(v) d v}{v-u-i \epsilon}=0$ or $1+\frac{(\Delta \omega)_{0}}{S_{\frac{1}{2}}}\left[\wp \int_{-\infty}^{\infty} \frac{\hat{\rho}(v) d v}{v-u}+i \pi \hat{\rho}(u)\right]^{2}=0$

(1) Given $\hat{\rho}(v)$ and pick a $u$

(2) Solve for $\frac{\operatorname{Re}(\Delta \omega)_{0}}{S_{\frac{1}{2}}}$ and $\frac{\operatorname{Im}(\Delta \omega)_{0}}{S_{\frac{1}{2}}}$

(3) Vary $u$ and trace out the contour.

One point on the contour is easy to solve. When $u=0, \wp \int=0 \longrightarrow$

$$
\frac{\operatorname{Re}(\Delta \omega)_{0}}{S_{\frac{1}{2}}}=0, \frac{\operatorname{Im}(\Delta \omega)_{0}}{S_{\frac{1}{2}}}=-\frac{1}{\pi \hat{\rho}(0)}
$$

This is intercept on vertical axis and is roughly $-1 / \sqrt{3}$

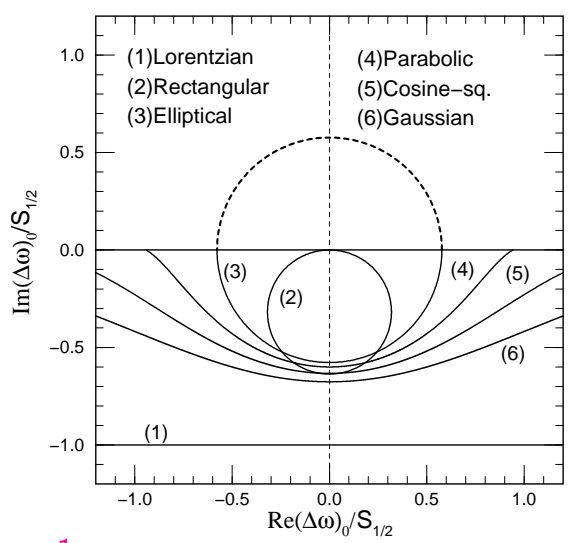

- Simplified stability criterion $\left|(\Delta \omega)_{0}\right|<\frac{1}{\sqrt{3}} S_{\frac{1}{2}} F_{\perp}$ $F_{\perp}=1.103,1,1.040,1.068,1.097,1.174$ for distr's (2), (3), (4), (5), (6) 


\section{Generalized elliptical distribution}

- $\hat{\rho}(v)=\frac{A_{n}}{a_{n}}\left(1-\frac{v^{2}}{a_{n}^{2}}\right)^{n} H\left(a_{n}-v\right)$

- By choosing various $n$ 's can reproduce nearly all distributions, from rectangular to Gaussian.

- Can approximate cosine-square with $n=2.36$

- Choose $A_{n}=\frac{\Gamma\left(n+\frac{3}{2}\right)}{\sqrt{\pi} \gamma(n+1)}$

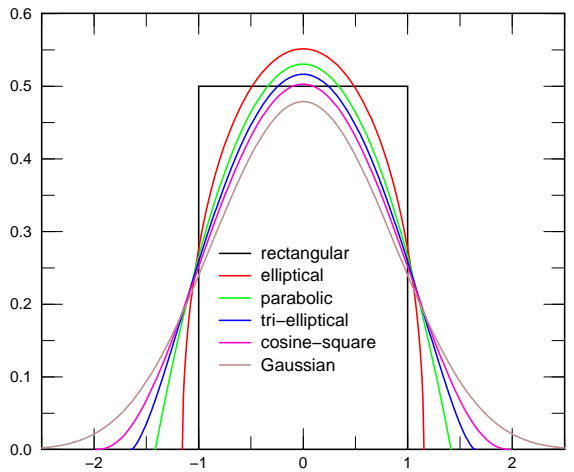
for normalization.

- Choose $a_{n}=\frac{1}{\sqrt{1-2^{-1 / n}}}$ so that $v=1$ is the HWHM.

- Trans. stability form factor $F_{\perp}=\frac{\sqrt{3} a_{n}}{\pi A_{n}}$

\begin{tabular}{lll}
\hline$n$ & distribution & $F_{\perp}$ \\
\hline 0 & rectangular & 1.103 \\
$\frac{1}{2}$ & elliptical & 1.000 \\
1 & parabolic & 1.040 \\
$\frac{3}{2}$ & tri-elliptical & 1.068 \\
2.36 & cosine square & 1.097 \\
$\infty$ & Gaussian & 1.174 \\
\hline
\end{tabular}




\section{Tune Spread Dependence on Momentum Spread}

- Spread in betatron tune can come from mom. spread: $\Delta \nu=\xi \delta$

- However, rev. freq. also has a spread from mom. spread: $\Delta \omega_{0}=-\eta \bar{\omega}_{0} \delta$

- Recall the dispersion relation $1+(\Delta \omega)_{0} \int \frac{\rho\left(\omega_{\beta}\right) d \omega_{\beta}}{\omega_{\beta}-\left(\Omega-n \omega_{0}\right)-i \epsilon}=0$

- $\omega_{\beta}-\left(\Omega-n \omega_{0}\right)=(n+\nu) \omega_{0}-\Omega=\left[(n+\nu) \omega_{0}-\overline{(n+\nu) \omega_{0}}\right]-\left[\Omega-\overline{(n+\nu) \omega_{0}}\right]$

$=\Delta\left[(n+\nu) \omega_{0}\right]-\Delta \Omega$

- $\Delta\left[(n+\nu) \omega_{0}\right]=\xi \delta \bar{\omega}_{0}-(n+\nu) \eta \bar{\omega}_{0} \delta=[\xi-\eta(n+\bar{\nu})] \delta \bar{\omega}_{0} \equiv \xi_{\text {eff }} \delta \bar{\omega}_{0}$

- Let $v=\frac{\delta}{\delta_{\frac{1}{2}}}, \quad u=\frac{\Delta \Omega}{\xi_{\text {eff }} \bar{\omega}_{0} \delta_{\frac{1}{2}}}$

chromaticity

eff. chromaticity

- Dispersion relation becomes $1+\frac{(\Delta \omega)_{0}}{\xi_{\text {eff }} \bar{\omega}_{0} \delta_{\frac{1}{2}}} \int \frac{\hat{\rho}(v) d v}{v-u+j \epsilon}$

$$
\xi_{\text {eff }} \bar{\omega}_{0} \delta_{\frac{1}{2}} \leftrightarrow S_{\frac{1}{2}}
$$

- Thus the stability contour will be exactly the same as before and the simplified stability criterion is $\left|\frac{(\Delta \omega)_{0}}{\xi_{\text {eff }} \bar{\omega}_{0} \delta_{\frac{1}{2}}}\right|<\frac{1}{\sqrt{3}} F_{\perp} \quad$ or $\quad\left|Z_{1}^{\perp}\right|<\frac{4 \pi \beta E_{0} \xi_{\text {eff }}}{\sqrt{3} e I_{0} \beta_{\perp}} \delta_{\frac{1}{2}} F_{\perp}$ 
Transverse Oscillation of a Point Bunch [10]

- A bunch will only be affected by its own wake turn-after-turn ago. $\ddot{y}(t)+\omega_{\beta}^{2} y(t)=-\frac{N e^{2}}{\gamma m C_{0}} \sum_{k=1}^{\infty}\left\langle y\left(t-k T_{0}\right)\right\rangle W_{1}\left(-k T_{0}\right)$

- Solve as initial-value problem in the freq. space by $\int_{0}^{\infty} \frac{d t}{2 \pi} e^{i \Omega t} \times \cdots$

- For the upper $\Omega$-plane $(\operatorname{Im} \Omega>0)$,

L.S. $=\left(-\Omega^{2}+\omega_{\beta}^{2}\right) \tilde{y}(\Omega)+\frac{\dot{y}_{0}-i \Omega y_{0}}{2 \pi}$ with $\tilde{y}(\Omega) \equiv \int_{-\infty}^{\infty} \frac{d t}{2 \pi} e^{i \Omega t} y(t)$

- R.S. $=-\frac{N e^{2}}{\gamma m C_{0}} \int \frac{d t}{2 \pi} e^{i \Omega t} \sum_{k}\left[\int d \omega e^{i \omega\left(t-k T_{0}\right)}\left\langle\tilde{y}\left(\omega^{\prime}\right)\right\rangle\right]\left[-i \beta \int \frac{d \omega^{\prime}}{2 \pi} Z_{1}^{\perp}\left(\omega^{\prime}\right) e^{i \omega^{\prime} k T_{0}}\right]$

- $\sum_{k} e^{-i \omega k T_{0}+i \omega^{\prime} k T_{o}}=2 \pi \sum_{p} \delta\left(\omega^{\prime} T_{0}-\omega T_{0}-2 \pi p\right)=\frac{2 \pi}{T_{0}} \sum_{p} \delta\left(\omega^{\prime}-\omega-p \omega_{0}\right)$

- $\int d t e^{i \Omega t-i \omega t}=2 \pi \delta(\omega-\Omega) \quad$ Next integrate over $d \omega^{\prime}$ and $d \omega$

- For $\operatorname{Im} \Omega>0, \quad\left(-\Omega^{2}+\omega_{\beta}^{2}\right) \tilde{y}(\Omega)-\frac{\dot{y}_{0}-i \Omega y_{0}}{2 \pi}=\frac{i \beta N e^{2} \mathcal{W}}{\gamma m C_{0}}\langle\tilde{y}(\Omega)\rangle$ 
- For the upper $\Omega$-plane $(\operatorname{Im} \Omega>0)$,

$\left(-\Omega^{2}+\omega_{\beta}^{2}\right) \tilde{y}(\Omega)-\frac{\dot{y}_{0}-i \Omega y_{0}}{2 \pi}=\frac{i \beta N e^{2} \mathcal{W}}{\gamma m C_{0}}\langle\tilde{y}(\Omega)\rangle \equiv-2 \bar{\omega}(\Delta \omega)_{0}\langle\tilde{y}(\Omega)\rangle$, where

$(\Delta \omega)_{0}=-\frac{i \beta N e^{2} \mathcal{W}}{2 \bar{\omega} \gamma m C_{0}}$ is freq. shift without Landau damping

and $\mathcal{W}=\frac{1}{T_{0}} \sum_{p} Z_{1}^{\perp}\left(p \omega_{0}+\Omega\right)$

- So problem is the same as that of

transverse oscillation of an unbunched beam.

The dispersion relation is exactly the same.

Only difference is the $(\Delta \omega)_{0}$. 


\section{Longitudinal Oscillation of an Extremely Short Bunch}

- Let $z$ be position of a particle ahead of some synchronous particle. $\frac{d z}{d s}=-\eta \delta, \quad \frac{d \delta}{d s}=\frac{\omega_{s}^{2}}{\eta v^{2}} z-\frac{N e^{2}}{\beta^{2} E_{0} C_{0}} \sum_{k} W_{0}^{\prime}\left[-k C_{0}-\left\langle z\left(s-k C_{0}\right)\right\rangle+z(s)\right]$

- Here we use $s$ as indep. variable, because time for a rev. varies due to syn. oscillation. $v$ is velocity of synchronous particle.

- $\frac{d^{2} z(s)}{d s^{2}}+\frac{\omega_{s}^{2}}{v^{2}} z(s)=\frac{\eta N e^{2}}{\beta^{2} E_{0} C_{0}} \sum_{k} W_{0}^{\prime}\left[-k C_{0}-\left\langle z\left(s-k C_{0}\right)\right\rangle+z(s)\right]$

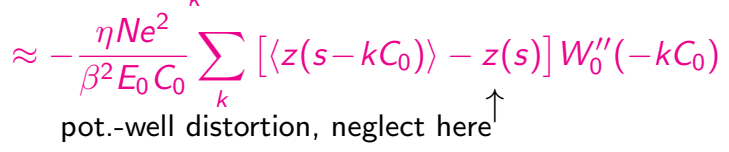

- Solve as initial-value problem in freq. space via $\int_{0}^{\infty} \frac{d s}{2 \pi} e^{i \Omega s / v} \times \cdots$.

- $\operatorname{Im} \Omega>0 \rightarrow \frac{\omega_{s}^{2}-\Omega^{2}}{v^{2}} \tilde{z}(\Omega)+\frac{z_{0}^{\prime}-i \Omega z_{0} / v}{2 \pi}=-\frac{i \eta N e^{2} \mathcal{W}}{\beta^{2} E_{0} C_{0}^{2}}\langle\tilde{z}(\Omega)\rangle \equiv-\frac{2 \bar{\omega}_{s}(\Delta \omega)_{0}}{v^{2}}\langle\tilde{z}(\Omega)\rangle$ where $(\Delta \omega)_{0}=\frac{i \eta N e^{2} \mathcal{W}}{2 \bar{\omega}_{s} \beta^{2} E_{0} T_{0}^{2}}$ is freq. shift without Landau damping and $\mathcal{W}=\sum_{p}\left(p \omega_{0}+\Omega\right) Z_{0}^{\|}\left(p \omega_{0}+\Omega\right)-\sum_{p} p \omega_{0} Z_{0}^{\|}\left(p \omega_{0}\right) \leftarrow \begin{gathered}\text { potential well } \\ \text { distortion }\end{gathered}$ 


\section{Robinsion Instabilities [16]}

- When imp. is a narrow resonance centered at $\omega_{r}$ near $q \omega_{0}$, only 2 syn. sidebands at $q \omega_{0} \pm \omega_{s}$ contribute.

- Without any spread in $\omega_{s}$ or Landau damping, growth rate is $\frac{1}{\tau}=\operatorname{Im}(\Delta \omega)_{0}=\frac{\eta N e^{2} \omega_{r}}{2 \omega_{s} \beta^{2} E_{0} T_{0}^{2}}\left[\operatorname{Re} Z_{0}^{\|}\left(q \omega_{0}+\omega_{s}\right)-\operatorname{Re} Z_{0}^{\|}\left(q \omega_{0}-\omega_{s}\right)\right]$

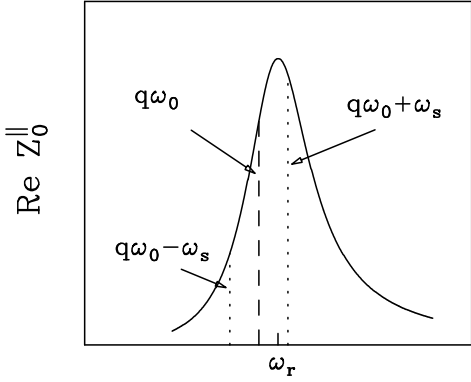

Angular Frequency

(a)

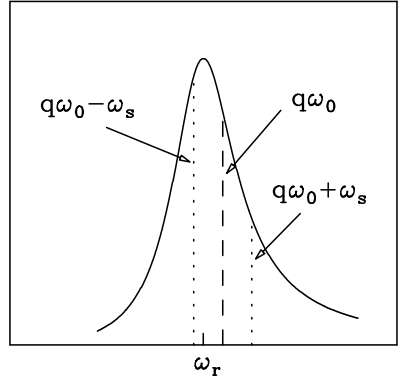

Angular Frequency

(b)

- Above transition $(\eta>0),(a)$ is anti-damped and (b) is damped. Need to detune $\omega_{r}$ to lower than $q \omega_{0}$ for stability. 
- It is clear that growth rate will be severe when resonance is very narrow.

- Example, $\eta=0.03, N=10^{11}, E_{0}=1 \mathrm{GeV}, \omega_{0}=9.4 \times 10^{6} \mathrm{~s}^{-1}, \nu_{\mathrm{s}}=0.01$,

$R_{s}=1 \mathrm{M} \Omega, h \omega_{0} / 2 \pi=360 \mathrm{MHz}, Q=2000$. If $\mathrm{rf}$ is detuned to

$\left(h \omega-\omega_{r}\right) / 2 \pi=-10 \mathrm{kHz}$,

growth time is $\tau=1.2 \mathrm{~ms}$, which is rather strong.

- For stability, detune lower above transition, detune higher below transition.

- Electron rings has rf cavities of relatively higher $Q$, and larger $\eta$; Robinson stability is an important issue.

- Proton rings have rf cavities of relatively lower $Q$ and smaller $\eta$; Robinson instability will be relative mild, even if cavities are detuned incorrectly in the wrong direction.

- For example, people were uncertain about the detuning of every rf cavity in the Tevatron at some time. 


\section{Transverse Robinson Instabilities}

- Recall that for a point bunch, the soln. of equation of motion is:

For the upper $\Omega$-plane $(\operatorname{Im} \Omega>0)$,

$$
\begin{aligned}
& \left(-\Omega^{2}+\omega_{\beta}^{2}\right) \tilde{y}(\Omega)-\frac{\dot{y}_{0}-i \Omega y_{0}}{2 \pi}=\frac{i \beta N e^{2} \mathcal{W}}{\gamma m C_{0}}\langle\tilde{y}(\Omega)\rangle \equiv-2 \bar{\omega}(\Delta \omega)_{0}\langle\tilde{y}(\Omega)\rangle \text {, where } \\
& (\Delta \omega)_{0}=-\frac{i \beta N e^{2}}{2 \bar{\omega} \gamma m C_{0} T_{0}} \sum_{p} Z_{1}^{\perp}\left(p \omega_{0}+\Omega\right)
\end{aligned}
$$

- So in the absence of Landau damping or spread in $\omega_{\beta}$, $\operatorname{Im}(\Delta \omega)_{0}$ is the growth rate.

- When the impedance is a narrow resonance near $q \omega_{0}$, only the sidebands $(q \pm[\nu]) \omega_{0}$ contribute.

Again stability is decided by whether the resonance peak $\omega_{r}$ is above or below $q \omega_{0}$.

- growth rate is

$$
\frac{1}{\tau}=\operatorname{Im}(\Delta \omega)_{0}=\frac{\beta N e^{2} c^{2} \beta_{\perp}}{2 E_{0} C_{0}^{2}}\left[\underset{\substack{\uparrow \\ \text { slow wave }}}{\operatorname{Re} Z_{1}^{\perp}}\left(p \omega_{0}-\omega_{\beta}\right)-\underset{\uparrow}{\operatorname{Re} Z_{1}^{\perp}}\left(p \omega_{0}+\omega_{\beta}\right)\right]
$$




\section{Longitudinal instabilities an unbunched beam}

- In the last 3 cases, there is an intrinsic oscillation to start with.

However, for an unbunched beam there is no syn. oscillation to start with The derivation of dispersion relation and stability criterion will be very much different.

- We start with a beam with uniform linear density $\lambda_{0}$ Let us envision a small perturbation $\lambda_{1}$ of harmonic $n$ and collective freq. $\Omega$ :

$\lambda(\theta, t)=\lambda_{0}+\lambda_{1} e^{-i(\Omega t-n \theta)}$.

$$
\frac{\lambda_{1}}{\lambda_{0}} \ll 1
$$

- Rev. freq. will also be perturbed:

$\omega(\theta, t)=\omega_{0}+\omega_{1} e^{-i(\Omega t-n \theta)}$.

- Continuity eq.: $\frac{\partial \lambda}{\partial t}+\frac{\partial}{\partial \theta}(\lambda \omega)=0 \longrightarrow \omega_{1}=\left(\frac{\Omega}{n}-\omega_{0}\right) \frac{\lambda_{1}}{\lambda_{0}}$.

- The current $I=\lambda \omega$ is also perturbed to

$$
I(\theta, t)=I_{0}+I_{1} e^{-i(\Omega t-n \theta)} \quad \text { with } I_{1}=\frac{\Omega \lambda_{1}}{n} . \quad\left\{\begin{array}{l}
\text { just linear density } \lambda_{1} \times \\
\text { angular velocity } \Omega / n
\end{array}\right.
$$

- $\therefore \lambda_{1}$ leads to the determination of $\omega_{1}$ and $l_{1}$. 
- $I_{1}$ interacts $Z_{0}^{\|}$, changes $E$ by $\frac{d E}{d t}=-\frac{e l_{1} Z_{0}^{\|}}{T_{0}}$, then $\omega$ by $\frac{d \omega}{d E}=-\frac{\eta \omega_{0}}{\beta^{2} E}$.

- Riding on a particle, one sees only the rev. freq. change due to energy change, or $\frac{d \omega}{d t}=\frac{\partial \omega}{\partial t}+\frac{\partial \omega}{\partial \theta} \frac{\partial \theta}{\partial t}=\frac{d \omega}{d E} \frac{d E}{d t}$.

- Thus $\frac{d \omega}{d t}=-i\left(\Omega-n \omega_{0}\right) \omega_{1}=\frac{\eta e l_{1} Z_{0}^{\|}}{2 \pi \beta^{2} E_{0}} \omega_{0}^{2}$,

$\omega_{1}=\left(\frac{\Omega}{n}-\omega_{0}\right) \frac{\lambda_{1}}{\lambda_{0}}$


- $I_{1}$ interacts $Z_{0}^{\|}$, changes $E$ by $\frac{d E}{d t}=-\frac{e l_{1} Z_{0}^{\|}}{T_{0}}$, then $\omega$ by $\frac{d \omega}{d E}=-\frac{\eta \omega_{0}}{\beta^{2} E}$.

- Riding on a particle, one sees only the rev. freq. change due to energy change, or $\frac{d \omega}{d t}=\frac{\partial \omega}{\partial t}+\frac{\partial \omega}{\partial \theta} \frac{\partial \theta}{\partial t}=\frac{d \omega}{d E} \frac{d E}{d t}$.

- Thus $\frac{d \omega}{d t}=-i\left(\Omega-n \omega_{0}\right) \omega_{1}=\frac{\eta e l_{1} Z_{0}^{\|}}{2 \pi \beta^{2} E_{0}} \omega_{0}^{2}, \quad \omega_{1}=\left(\frac{\Omega}{n}-\omega_{0}\right) \frac{\lambda_{1}}{\lambda_{0}}$

- Physical meaning:

(1) $I_{1}$ interacts with $Z_{0}^{\|}$to give $V=I_{1} Z_{0}^{\|}$, creating $n$ buckets along the ring.

(2) Inside a bucket, executes syn. freq.: $\omega_{s}^{2}=\frac{i \eta n e l_{1} Z_{0}^{\|}}{2 \pi \beta^{2} E_{0}} \omega_{0}^{2}$. $-\frac{\eta h e V_{\mathrm{RF}} \cos \phi_{s}}{2 \pi \beta^{2} E_{0}}$

(3) Voltage and current are $90^{\circ}$ out of phase, therefore the factor $i$

(4) Particle inside a bucket moves azimuthal angle $\Delta \theta=\frac{\pi}{n}$ in $\Delta t=\frac{\pi}{\omega_{s}}$, or a perturbed rev. freq. $\Delta \omega \sim \frac{\omega_{s}}{n} e^{-i \omega_{s} t}$ bucket width $2 \pi / n$.

(5) Thus $\frac{d \omega}{d t}=-i \frac{\omega_{s}^{2}}{n}$. 
- $I_{1}$ interacts $Z_{0}^{\|}$, changes $E$ by $\frac{d E}{d t}=-\frac{e l_{1} Z_{0}^{\|}}{T_{0}}$, then $\omega$ by $\frac{d \omega}{d E}=-\frac{\eta \omega_{0}}{\beta^{2} E}$.

- Riding on a particle, one sees only the rev. freq. change due to energy change, or $\frac{d \omega}{d t}=\frac{\partial \omega}{\partial t}+\frac{\partial \omega}{\partial \theta} \frac{\partial \theta}{\partial t}=\frac{d \omega}{d E} \frac{d E}{d t}$.

- Thus $\frac{d \omega}{d t}=-i\left(\Omega-n \omega_{0}\right) \omega_{1}=\frac{\eta e l_{1} Z_{0}^{\|}}{2 \pi \beta^{2} E_{0}} \omega_{0}^{2}$,

- Physical meaning:

(1) $I_{1}$ interacts with $Z_{0}^{\|}$to give $V=l_{1} Z_{0}^{\|}$, creating $n$ buckets along the ring.

(2) Inside a bucket, executes syn. freq.: $\omega_{s}^{2}=\frac{i \eta n e l_{1} Z_{0}^{\|}}{2 \pi \beta^{2} E_{0}} \omega_{0}^{2} \cdot-\frac{\eta h e V_{\mathrm{RF}} \cos \phi_{s}}{2 \pi \beta^{2} E_{0}}$

(2) Inside a bucket, executes syn. freq.: $\omega_{s}^{2}=\frac{i \eta n e l_{1} Z_{0}^{\|}}{2 \pi \beta^{2} E_{0}} \omega_{0}^{2} \cdot \frac{\eta h e V_{\mathrm{RF}} \cos \phi_{s}}{2 \pi \beta^{2} E_{0}}$
(3) Voltage and current are $90^{\circ}$ out of phase, therefore the factor $i$

(4) Particle inside a bucket moves azimuthal angle $\Delta \theta=\frac{\pi}{n}$ in $\Delta t=\frac{\pi}{\omega_{s}}$, or a perturbed rev. freq. $\Delta \omega \sim \frac{\omega_{s}}{n} e^{-i \omega_{s} t}$

$\omega_{1}=\left(\frac{\Omega}{n}-\omega_{0}\right) \frac{\lambda_{1}}{\lambda_{0}}$

(5) Thus $\frac{d \omega}{d t}=-i \frac{\omega_{s}^{2}}{n}$.

- From above, $\left(\Omega-n \omega_{0}\right)^{2}=\frac{i n \eta e l_{1} Z_{0}^{\|}}{2 \pi \beta^{2} E_{0}} \frac{\lambda_{0}}{\lambda_{1}} \omega_{0}^{2}=\frac{i n \eta e I_{0} Z_{0}^{\|}}{2 \pi \beta^{2} E_{0}} \omega_{0}^{2}$. 
- Finally $(\Delta \omega)_{0}^{2} \equiv\left(\Omega-n \omega_{0}\right)^{2}=\frac{i n \eta I_{0} Z_{0}^{\|}}{2 \pi \beta^{2} E_{0}} \omega_{0}^{2}$.

- We identify $(\Delta \omega)_{0}$ as shift in rev. freq. due to $Z_{0}^{\|}$in the absence of Landau damping.

- Dispersion relation is slightly different: $1-(\Delta \omega)_{0}^{2} \int_{C} \frac{\rho\left(\omega_{0}\right) d \omega_{0}}{\left(\Omega-n \omega_{0}\right)^{2}}=0$.

- Landau damping comes from variation of $\omega_{0}$ inside the unbunched beam.

- Let $v=\frac{\omega_{0}-\bar{\omega}_{0}}{S_{\frac{1}{2}}}, \hat{\rho}(v)=S_{\frac{1}{2}} \rho\left(\omega_{0}\right)$ so that $\int \hat{\rho}(v) d v=1$ and $v=1$ is at HWHM.

- Further let $u=\frac{\Omega / n-\bar{\omega}_{0}}{S_{\frac{1}{2}}}$.

Dispersion relation becomes $1-\frac{(\Delta \omega)_{0}^{2}}{n^{2} S_{\frac{1}{2}}^{2}} \int_{C} \frac{\hat{\rho}(v) d v}{(v-u)^{2}}=0$.

- Integration by parts: $1-\frac{(\Delta \omega)_{0}^{2}}{n^{2}{S_{\frac{1}{2}}^{2}}^{2}} \int_{C} \frac{\hat{\rho}^{\prime}(v) d v}{v-u}=0$. 
- To obtain the stability contour, let $u \rightarrow u+i \epsilon$, with $u$ real and $\epsilon=0^{+}$.

Then what needs to be solve is $1-\frac{(\Delta \omega)_{0}^{2}}{n^{2} S_{\frac{1}{2}}^{2}} \int_{C} \frac{\hat{\rho}^{\prime}(v) d v}{v-u-i \epsilon}=0$

- Spread in $\omega_{0}$ usually comes from spread in momentum $\delta$, then $S_{\frac{1}{2}}=|\eta| \bar{\omega}_{0} \delta_{\frac{1}{2}}$.

- Finally obtain $1-\frac{2 i \operatorname{sgn}(\eta)}{\pi}(U+i V) \int \frac{\hat{\rho}^{\prime}(v) d v}{v-u-i \epsilon}=0$ with $U+i V=\frac{e l_{0}}{4|\eta| \beta^{2} E_{0} \delta_{\frac{1}{2}}^{2}} \frac{Z_{0}^{\|}\left(n \bar{\omega}_{0}\right)}{n}, \quad v=\frac{\delta}{\delta_{\frac{1}{2}}}, \quad u=\frac{1}{|\eta| \delta_{\frac{1}{2}}}\left(\frac{\Omega}{n \omega_{0}}-1\right)$

- $1-\frac{2 i \operatorname{sgn}(\eta)}{\pi}(U+i V)\left[\wp \int \frac{\hat{\rho}^{\prime}(v) d v}{v-u}+i \pi \hat{\rho}^{\prime}(u)\right]=0$.

- One point is easy to solve: $\hat{\rho}^{\prime}(u)=0$ when $u=0$

This is intercept on $V$-axis:

$$
U=0 \quad \text { and } \quad 1+\frac{2 \operatorname{sgn}(\eta) V}{\pi} \wp \int \frac{\hat{\rho}^{\prime}(v) d v}{v}=0
$$

$$
(\Delta \omega)_{0}^{2}=\frac{i n \eta I_{0} Z_{0}^{\|}}{2 \pi \beta^{2} E_{0}} \omega_{0}^{2}
$$




\section{Stability Contours}

- Stability plot for $\eta<0$ : most contours cut $V$-axis at $V=-1$.

- Simplified stability criterion

$$
\left|\frac{Z_{0}^{\|}}{n}\right|<\frac{4|\eta| \beta^{2} E_{0}}{e l_{0}} \delta_{\frac{1}{2}}^{2} F_{\|}
$$

- For generalized elliptical model $\hat{\rho}(v)=\frac{A_{n}}{a_{n}}\left[1-\frac{v^{2}}{a_{n}^{2}}\right]^{n}, \quad F_{\|}=\frac{\pi a_{n}^{2}}{4 n+2}$

- For distributions with long tails, system is stable below transition if $Z_{0}^{\|}$is pure capacitive.

- As soon as transition is crossed, capacitive imp. excites instability because of small $\eta$

- negative-mass instability

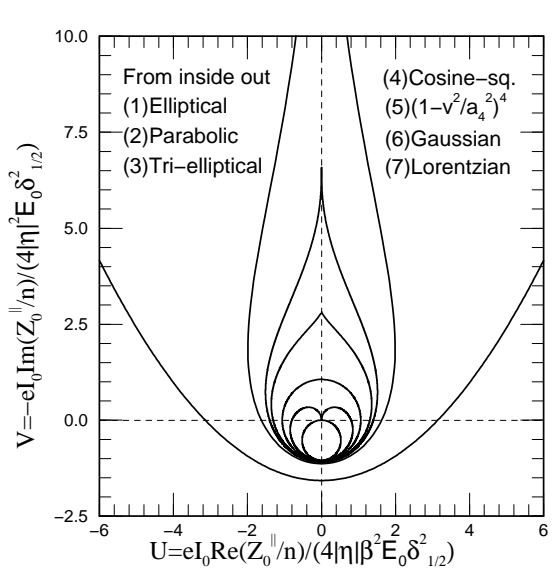




\section{Comments}

- For Gaussian distribution, the stability contour can be integrated in the closed form with $F_{\|}=\frac{\pi}{4 \ln 2}=1.133$. Stability criterion can

also be expressed neatly as $\left|\frac{Z_{0}^{\|}}{n}\right|<\frac{|\eta| \beta^{2} E_{0}}{e l_{0}} \sigma_{\delta}^{2}$

- Although for unbunched beam, criterion can be applied to long bunches provided that (suggestion by Boussard) [14]

1 . The perturbing wavelength $\lambda_{1} \ll$ bunch length

2. Growth time $\tau \ll T_{s}$, syn. period

3. Substituting average current $I_{0}$ by peak local current $I_{\mathrm{pk}}$.

- Microwave instability is not catastrophic.

When instability occurs, bunch will be lengthened and mom. spread increased so that stability is re-established. 
- Since the growth is fast and stability becomes re-established, it poses difficulty in observing the microwave signals.

- If a network analyzer is used to view the bunch spectrum, high-freq. signals will often not be observed, because it takes time for the analyzer to process high freq. signals.

- Analyzer must be set at a narrow freq. span or zero span, and observe beam signal as a fcn. of time.

The freq. span is next set to an adjacent narrow freq. interval and the observation repeated. 
Microwave Signal Observation

- Pick-up signals after injection in CERN ISR [13]

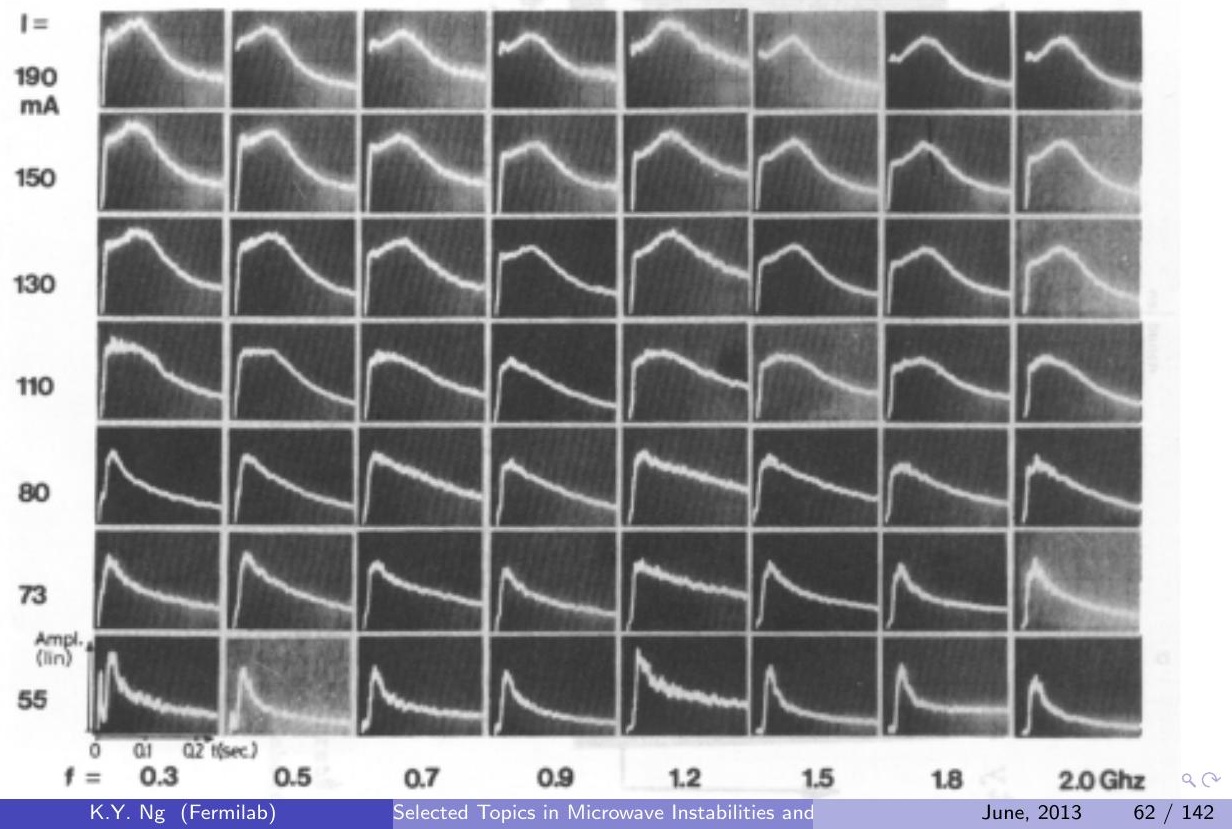




\section{Determination of $Z_{0}^{\|}$from Instability [14]}

- A very thick cable has to be used from beam monitor to network analyzer, because signal decays over distance.

- RF voltage lowered adiabatically $\Longrightarrow$ mom. spread will be reduced gradually until stability criterion is violated. Usually, it is hard to know the exact $V_{\mathrm{rf}}$ when it is low enough.

- RF voltage turned off abruptly, beam will be debunched. When local mom. spread is small enough, instability occurs.

- RF cavity must be mechanically shorted to avoid beam loading.

- It is hard to determine exact time when stability occurs.

- Fig: CERN CPS, signals between 1.5 to $1.8 \mathrm{GHz}, 2 \mathrm{~ms} /$ division

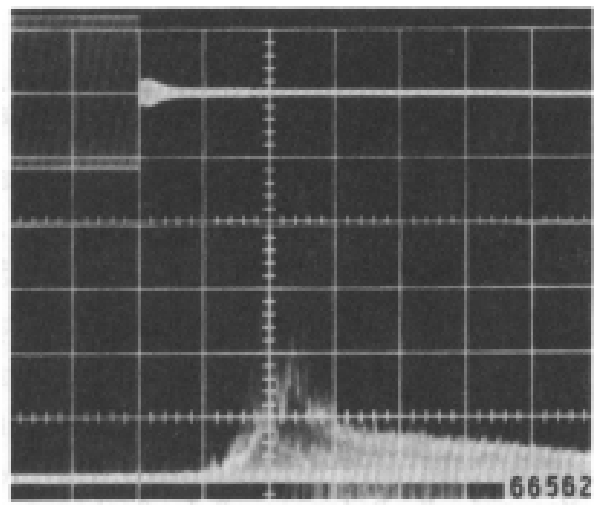




\section{Longitudinal Decoherence}

- Dispersion relation for microwave instability depends on

gradient of energy or frequency distribution, $\frac{d f_{0}(\Delta E)}{d \Delta E}$ or $\frac{d f_{0}\left(\Delta \omega_{0}\right)}{d \Delta \omega_{0}}$

- Thus flat distribution $\longrightarrow$ no Landau damping.

- Is there longitudinal decoherence for a flat beam?

- We study the response of an energy impulse to a beam at $t=0$. [21]

- At $t=0+, f(\theta, \Delta E ; 0+)=f_{0}(\Delta E-\widehat{\delta E} \cos k \theta)=f_{0}(\Delta E)-\frac{d f_{0}}{d \Delta E} \widehat{\delta E} \cos k \theta$

- A particle moves according to $\theta=\theta_{0}+\omega_{0} t$,

Perturbed distribution at time is $f_{1}(\theta, \Delta E ; t)=-\frac{d f_{0}}{d \Delta E} \widehat{\delta E} \cos \left(k \theta-k \omega_{0} t\right)$.

- Perturbed part of current jumps from zero to

$I_{1}(\theta, t)=\frac{e N}{2 \pi} \int \omega_{0}(\Delta E) f_{1}(\theta, \Delta E ; t) d \Delta E$ 
- $I_{1}(\theta, t)=\underbrace{-H(t) \frac{e N \widehat{\delta E}}{2 \pi} \int \frac{d f_{0}}{d \Delta E} \omega_{0} \cos \left(k \theta-k \omega_{0} t\right) d \Delta E} \quad H(t)= \begin{cases}1 & t>0 \\ 0 & t<0\end{cases}$

$\uparrow$ Response function

- So longitudinal decoherence does depends on gradient of distribution.

- Next introduce $\Delta \omega_{0}=\omega_{0}-\bar{\omega}_{0}$ and $g_{0}\left(\Delta \omega_{0}\right)$.

Then $f_{0}(\Delta E) d \Delta E=g_{0}\left(\Delta \omega_{0}\right) d \Delta \omega_{0}$

and $\frac{d f_{0}}{d \Delta E} d \Delta E=\frac{d g_{0}}{d \Delta E} d \Delta \omega_{0}=-\frac{\eta \bar{\omega}_{0}}{\beta^{2} E_{0}} \frac{d g_{0}}{d \Delta \omega_{0}} d \Delta \omega_{0}$

- $I_{1}(\theta, t)=\frac{e N \eta \bar{\omega}_{0}^{2} \widehat{\delta E}}{2 \pi \beta^{2} E_{0}} \int_{-\infty}^{\infty} \frac{d g_{0}}{d \Delta \omega_{0}}\left[\cos \left(k \theta-k \bar{\omega}_{0} t\right) \cos k \Delta \omega_{0} t\right.$

$$
\left.+\sin \left(k \theta-k \bar{\omega}_{0} t\right) \sin k \Delta \omega_{0} t\right] d \Delta \omega_{0}
$$

- If $g_{0}\left(\Delta \omega_{0}\right)$ is even,

$$
I_{1}(\theta, t)=\frac{e N \eta \bar{\omega}_{0}^{2} \widehat{\delta E}}{2 \pi \beta^{2} E_{0}} \sin \left(k \theta-k \bar{\omega}_{0} t\right) \int_{-\infty}^{\infty} \frac{d g_{0}}{d \Delta \omega_{0}} \sin k \Delta \omega_{0} t d \Delta \omega_{0} .
$$




\section{About the Plots}

- $f(\theta, \Delta E ; t)=f_{0}\left[\Delta E-\widehat{\delta E} \cos \left(k \theta-k \bar{\omega}_{0} t+\frac{k \eta \bar{\omega}_{0} t}{\beta^{2} E_{0}} \Delta E\right)\right]$

- Normalized everything to the rms energy spread $\sigma_{E}$

- $f(\theta, \Delta E ; t)=f_{0}[\varepsilon-a \cos (k \theta+k \xi \varepsilon)]$

where $\varepsilon=\frac{\Delta E}{\sigma_{E}}, \quad a=\frac{\widehat{\delta E}}{\sigma_{E}}, \quad \xi=\frac{2 \pi n \eta \bar{\sigma}_{E}}{\beta^{2} E_{0}}$

- Equi-density curve at density $x \sigma^{\prime} s f_{0}(x)$ is obtained by solving for $\varepsilon(\theta)$ from $\quad \varepsilon-a \cos (k \theta+k \xi \varepsilon)=x$

- Initial distribution: $f_{0}(x)=\frac{1}{\sqrt{2 \pi}} e^{-x^{2} / 2} \longleftarrow$ Gaussian assumed

- Perturbed current: $I(\theta, \xi)=\int_{-\infty}^{\infty} f_{0}[\varepsilon-a \cos (k \theta+k \xi \varepsilon)] d \varepsilon$ 


\section{Transition}

- For a particle in an accelerator ring, the period is $T=\frac{C}{\mathrm{~V}}$

Relative to the synchronized particle, $\frac{\Delta T}{T_{0}}=\frac{\Delta C}{C_{0}}-\frac{\Delta v}{v_{0}}$

$$
\text { or } \frac{\Delta T}{T_{0}}=\left(\alpha-\frac{1}{\gamma^{2}}\right) \frac{\Delta p}{p_{0}}=\eta \frac{\Delta p}{p_{0}}
$$

- Transition is crossed when frequency-slip parameter $\eta=0$.

- Above transition, velocity decreases as more energy is put in, as if the mass is negative.

- Space-charge force is repulsive. But above transition, it is attractive. Particles tend to lump together at the same place.

This is called negative-mass instability.

- Negative-mass instability is a type of microwave instability, which will also result in emittance growth and even possible breakup of the bunch.

- Instability occurs only when $|\eta|$ is small (close to transition).

- It is important to know the growth of emittance. 


\section{Negative-Mass Instability}

- Growth rate without Landau damping at peak current $I_{p}$ at the revolution harmonic $k_{c}$ is $G\left(k_{c}, t\right)=\omega_{0}\left(\frac{\eta e k_{c} Z_{I} I_{p}}{2 \pi \beta^{2} \gamma E_{0}}\right)^{1 / 2}$ where $Z_{I}$ is $\left.\operatorname{Im} Z_{0}^{\|}\right|_{\mathrm{spch}}=\frac{Z_{I}}{k_{c}}=\frac{Z_{0} g}{2 \beta \gamma^{2}}$

- At low freq., geometric factor $g \rightarrow g_{0}=1+2 \ln \frac{b}{a}$, where $a$ is beam radius and $b$ is beam pipe radius.

- Thus the growth rate increases linearly with harmonic $k_{c}$.

- But at high harmonic, $g$ rolls off at high frequencies roughly like

$$
g=\frac{g_{0}}{1+\left(k_{c} / k_{c \frac{1}{2}}\right)^{2}} \quad \frac{b}{a} \text { not too big, } \quad k_{c \frac{1}{2}} \approx \gamma R\left(\frac{1.6}{b}+\frac{0.52}{a}\right)
$$

- Therefore $Z_{I} / k_{c}$ is almost constant below $k_{c \frac{1}{2}}$ and the growth rate is $G\left(k_{c}, t\right) \propto k_{c}$.

- With Landau damping, Hardt showed that the growth rate becomes $G\left(k_{c}, t\right) \propto k_{c} g^{2}$ with maximum at $k_{c p}=k_{c \frac{1}{2}} / \sqrt{3}$. 


\section{Fermilab Old Main Ring}

- Fermilab Main Ring with $a=5 \mathrm{~mm}$ and $b=35 \mathrm{~mm}$ : this corresponds to $77.6 \mathrm{GHz}$.

- But cutoff frequency is $\sim 1.5 \mathrm{GHz}$.

- Typical cycle: Growth of power spectral line is $1.5 \times 10^{6}$ times at $77.6 \mathrm{GHz}$ and 1.6 times at $1.5 \mathrm{GHz}$.

- In Wei's simulation, [22] the bunch was divided into bins with bin width equal to the cutoff wavelength, so higher frequencies were not included.

- Lee and Teng [23] were the first to demonstrate negative-mass instability by simulating transition crossing in the Fermilab Booster.

They divided the bunch into cutoff wavelengths also.

- Lee said that as the bins become smaller, the observed growth is larger. But because of limited speed of computer in the old days, he could not reduce bin size by too much. 


\section{Schottky-Noise Model}

- Hardt [24] assumed that the seeds of the negative-mass growth come from statistical fluctuations or Schottky noise of the finite number of particles $N_{b}$ within the bunch.

- The growth across transition is to be derived analytically.

- We measure rf phase offset $\Delta \phi$ from the synchronous particle.

- The bunch is supposed to have an expected smooth distribution $F(\Delta \phi)$, which is normalized to $2 \widehat{\Delta \phi}$, the total bunch length.

In other words, $\frac{1}{2 \widehat{\Delta \phi}} \int F(\Delta \phi) d \Delta \phi=1$.

- The bunch is divided into $M$ bins in the rf phase

- Number of particles in $m$ th bin is $\frac{N_{b} F\left(\Delta \phi_{m}\right)}{M}$.

- Due to statistical fluctuation, the $m$ th bin contains $\delta N_{m}$ extra particles.

- Define a fluctuation step function: $f(\Delta \phi, t)=\frac{\delta N_{m}}{\Delta N}$ where $\Delta N=\frac{N_{b}}{M}$ is average number of particles in a bin. 
- Expand in a Fourier series $f(\Delta \phi, t)=\sum_{k_{b}=-\infty}^{\infty} c_{k_{b}}(t) e^{i 2 \pi k_{b} \Delta \phi /(2 \widehat{\Delta \phi})}$ where $c_{k_{b}}(t)=\frac{1}{2 \widehat{\Delta \phi}} \int_{-\widehat{\Delta \phi}}^{\widehat{\Delta \phi}} f(\Delta \phi, t) e^{-i 2 \pi k_{b} \Delta \phi /(2 \widehat{\Delta \phi})} d \Delta \phi$

- In above, $k_{b}$ is bunch mode number, or number of wavelengths can reside in the bunch.

We are using periodic boundary conditions. Therefore $k_{b}$ can be all integers, positive and negative.

Relation of $k_{b}$ to harmonic number $n$ or $k_{c}$ is $\frac{k_{b}}{k_{c}}=\frac{2 \widehat{\Delta \phi}}{2 \pi h}$.

- $E\left[\left|c_{k_{b}}(0)\right|^{2}\right]=\frac{1}{(2 \widehat{\Delta \phi})^{2}} \int_{-\widehat{\Delta \phi}}^{\widehat{\Delta \phi}} d \Delta \phi \int_{-\widehat{\Delta \phi}}^{\widehat{\Delta \phi}} d \Delta \phi^{\prime} E\left[\frac{\delta N_{m} \delta N_{n}}{(\Delta N)^{2}}\right] e^{i 2 \pi k_{b}\left(\Delta \phi-\Delta \phi^{\prime}\right) /(2 \widehat{\Delta \phi})}$

- Since $E\left[\delta N_{m} \delta N_{n}\right]=\delta_{m n} \Delta N F(\Delta \phi)$,

$E\left[\left|c_{k_{b}}(0)\right|^{2}\right]=\frac{1}{(2 \widehat{\Delta \phi})^{2}} \int_{-\widehat{\Delta \phi}}^{\widehat{\Delta \phi}} \frac{F(\Delta \phi)}{\Delta N} \frac{2 \widehat{\Delta \phi}}{M} d \Delta \phi=\frac{1}{N_{b}}$

independent of mode number $k_{b}$ and the number of bins $M$, otherwise model will be meaningless. 
- The growth rate of each mode amplitude $c_{k_{b}}$ can be derived from Vlasov equation, and the evolution is $\left|c_{k_{b}}(t)\right| \approx \frac{1}{\sqrt{N_{b}}} \exp \int_{0}^{t} G\left(k_{c}, t\right) d t$

- Hardt's assertion: no blowup if $\sum_{k_{b}}\left|c_{k_{b}}\left(t_{0}\right)\right|^{2}<1$ where $t_{0}$ is end of nonadiabatic time or when stability is regained.

- Comment 1: Critical condition implies $\frac{1}{2 \widehat{\Delta \phi}} \int\left|f\left(\Delta \phi, t_{0}\right)\right|^{2} d \Delta \phi=1$ or the average fluctuation in each bin is comparable to the average number of particles in each bin.

However, below critical condition, there is still emittance blowup.

- Comment 2: Perturbation expansion is $F(\Delta \phi)+f(\Delta \phi, t)=F(\Delta \phi)+\sum_{k_{b}=-\infty}^{\infty} c_{k_{b}}(t) e^{i 2 \pi k_{b} \Delta \phi /(2 \widehat{\Delta \phi})}$

The perturbation is justified when $\sum_{k_{b}}\left|c_{k_{b}}\left(t_{0}\right)\right|^{2}<1$.

- Remaining problem: compute $G\left(k_{c}, t\right)$ in the presence of Landau damping. 


\section{Dispersion Relation}

- To obtain growth rate in the presence of Landau damping, we need to solve the dispersion relation

$$
1=-\left(\frac{\Delta \Omega_{1}}{n}\right)^{2} \int \frac{d F(\Delta \omega) / d \Delta \omega}{\Delta \Omega / n-\Delta \omega} d \Delta \omega
$$

where the frequency shift without Landau damping is

$$
\left(\frac{\Delta \Omega_{1}}{n}\right)^{2}=\frac{i e l_{\text {local }} \eta \omega_{0}^{2}\left[Z_{0}^{\|}(\Omega) / n\right]_{\mathrm{spch}}}{2 \pi \beta^{2} \gamma E_{\text {rest }}}
$$

- What Hardt did:

1. Assume elliptical initial distribution

$$
\psi(\Delta \phi, \Delta E)=\frac{3}{2 \pi \widehat{\Delta \phi} \widehat{\Delta E}} \sqrt{1-\frac{\Delta \phi^{2}}{\widehat{\Delta \phi}^{2}}-\frac{\Delta E^{2}}{\widehat{\Delta E}^{2}}}
$$

which becomes parabolic in linear distribution.

2. The region of maximum growth is center of bunch.

3. Substitute in dispersion relation, and solve for $\operatorname{Im} \Delta \Omega$. 


\section{Analytic Solution}

- Growth per unit $x=t / T_{c}$

$$
G(n, x)=T_{c} \operatorname{Im} \Omega=\frac{n \eta_{N}}{h} \sqrt{\frac{S_{c}\left|\tan \phi_{s}\right| \beta_{t}}{\pi \dot{\gamma}_{t} T_{c}}} \frac{1-\frac{x}{\eta_{N} \theta}}{\sqrt{\frac{2 \eta_{N} \theta}{x}-1}}
$$

$T_{C}$ is nonadiabatic time

$\eta_{N} \propto g(n)$ is space charge parameter

$S_{c}=\pi \widehat{\beta \gamma} \widehat{\Delta \phi}$ is long bunch emittance

$\theta$ is normalized half bunch length

$\phi_{s}$ is synchronous if phase

- The growth increases from zero at $x=0$ to a maximum and decreases to zero at $x=\eta_{N} \theta$.

- Integrated growth decrement

$$
E_{\mathrm{acc}}(n)=\int_{0}^{\eta_{N} \theta} G(n, x) d x=\frac{n \eta_{N}^{2} \theta}{h}\left(1-\frac{\pi}{4}\right) \sqrt{\frac{S_{c}\left|\tan \phi_{s}\right| \beta_{t}}{\pi \dot{\gamma}_{t} T_{c}}}
$$


- Recall that the space charge geometric factor $g(n)$ rolls off at high freq.

- $E_{\text {acc }}(n) \propto \frac{n}{\left(1+\frac{n^{2}}{n_{\frac{1}{2}}^{2}}\right)^{2}}$

- The maximum occurs at $n=n_{\max }=n_{\frac{1}{2}} / \sqrt{3}$ :

$$
E_{\max }=\frac{3 \sqrt{3} n_{\frac{1}{2}} \eta_{N 0}^{2} \theta}{16 h}\left(1-\frac{\pi}{4}\right) \sqrt{\frac{S_{c}\left|\tan \phi_{s}\right| \beta_{t}}{\pi \dot{\gamma}_{t} T_{c}}}
$$

- Up to now, we have everything derived to compute the growth from Schottky noise, i.e.,

$$
\begin{aligned}
& f(\Delta \phi, t)=\sum_{k_{b}=-\infty}^{\infty} c_{k_{b}}(t) e^{i 2 \pi k_{b} \Delta \phi /(2 \widehat{\Delta \phi})} \\
& \left|c_{k_{b}}(t)\right| \approx \frac{1}{\sqrt{N_{b}}} \exp \int_{0}^{t} G\left(k_{c}, t\right) d t=\frac{1}{\sqrt{N_{b}}} \exp E_{\text {acc }}\left(k_{b}\right)
\end{aligned}
$$

- In a linac, we can follow this idea to compute the microwave instability growth with Schottky noise as seeds. 


\section{Final Touch of Transition Growth}

- Hardt's blowup assertion:

$$
\sum_{k_{b}=-\infty}^{\infty}\left|c_{k_{b}}\left(t_{0}\right)\right|^{2} \lesssim 1 \Longrightarrow \sum_{k_{b}=-\infty}^{\infty} \exp \left[2 E_{\mathrm{acc}}\left(k_{b}\right)\right] \lesssim N_{b}
$$

- Because of sharp peak at $n_{\max }=n_{\frac{1}{2}} / \sqrt{3}$, can transform $\sum_{k_{b}}$ to an integral and employ method of steepest descend for evaluation.

- Final result: $E_{\mathrm{crit}} \approx \frac{1}{2}\left[\ln N_{b}-\ln \left(\frac{2 k_{b \frac{1}{2}}}{3} \sqrt{\frac{\pi}{\ln N_{b}}}\right)\right]$

- Can introduce a parameter c: [25]

$E_{\text {acc }}=c E_{\text {crit }} \quad$ or

$2.45 n_{\max }\left(\frac{r_{p}}{R}\right)^{2}\left(\frac{E_{\mathrm{rest}}^{5 / 2} \beta_{t}^{7 / 6}}{h^{1 / 3} \omega_{0}^{4 / 3} \gamma_{t}^{2 / 3}}\right)\left(\frac{N_{b}^{2} g_{0}^{2}\left|\tan \phi_{s}\right|^{1 / 3}}{S^{5 / 2} \dot{\gamma}_{t}^{7 / 6}}\right)=c E_{\mathrm{crit}}$

Thus $c<1$ implies no blowup. 


\section{Numerical Results}

\begin{tabular}{ccrrrr}
\hline $\begin{array}{c}\text { Bunch area } \\
(\mathrm{eV}-\mathrm{s})\end{array}$ & Half bunch width & \multicolumn{2}{c}{$N_{b}=2.2 \times 10^{10}$} & \multicolumn{2}{c}{$N_{b}=4.0 \times 10^{10}$} \\
& $(\mathrm{~ns})$ & $c$ & $E_{\text {crit }}$ & \multicolumn{1}{c}{$c$} & $E_{\text {crit }}$ \\
\hline 0.040 & 0.439 & 3.84 & 10.23 & 12.70 & 10.54 \\
0.050 & 0.490 & 2.21 & 10.18 & 7.31 & 10.48 \\
0.060 & 0.537 & 1.41 & 10.13 & 4.65 & 10.44 \\
0.070 & 0.580 & 0.96 & 10.09 & 3.18 & 10.40 \\
0.080 & 0.620 & 0.69 & 10.06 & 2.28 & 10.36 \\
0.100 & 0.693 & 0.40 & 10.00 & 1.31 & 10.31 \\
0.120 & 0.760 & 0.25 & 9.96 & 0.84 & 10.26 \\
0.140 & 0.820 & 0.17 & 9.92 & 0.57 & 10.22 \\
0.160 & 0.877 & 0.12 & 9.89 & 0.41 & 10.19 \\
0.180 & 0.930 & 0.09 & 9.86 & 0.31 & 10.16 \\
0.200 & 0.981 & 0.07 & 9.83 & 0.24 & 10.13 \\
0.220 & 1.028 & 0.06 & 9.81 & 0.19 & 10.11 \\
0.240 & 1.074 & 0.05 & 9.78 & 0.15 & 10.09 \\
\hline
\end{tabular}

Critical parameter $c$ for negative-mass instability for a proton bunch in the Fermilab Main Ring with $N_{b}=2.2 \times 10^{10}$ or $4.0 \times 10^{10}$ particles.

The ramp rate across transition is $\dot{\gamma}_{t}=90.0 \mathrm{~s}^{-1}$. 

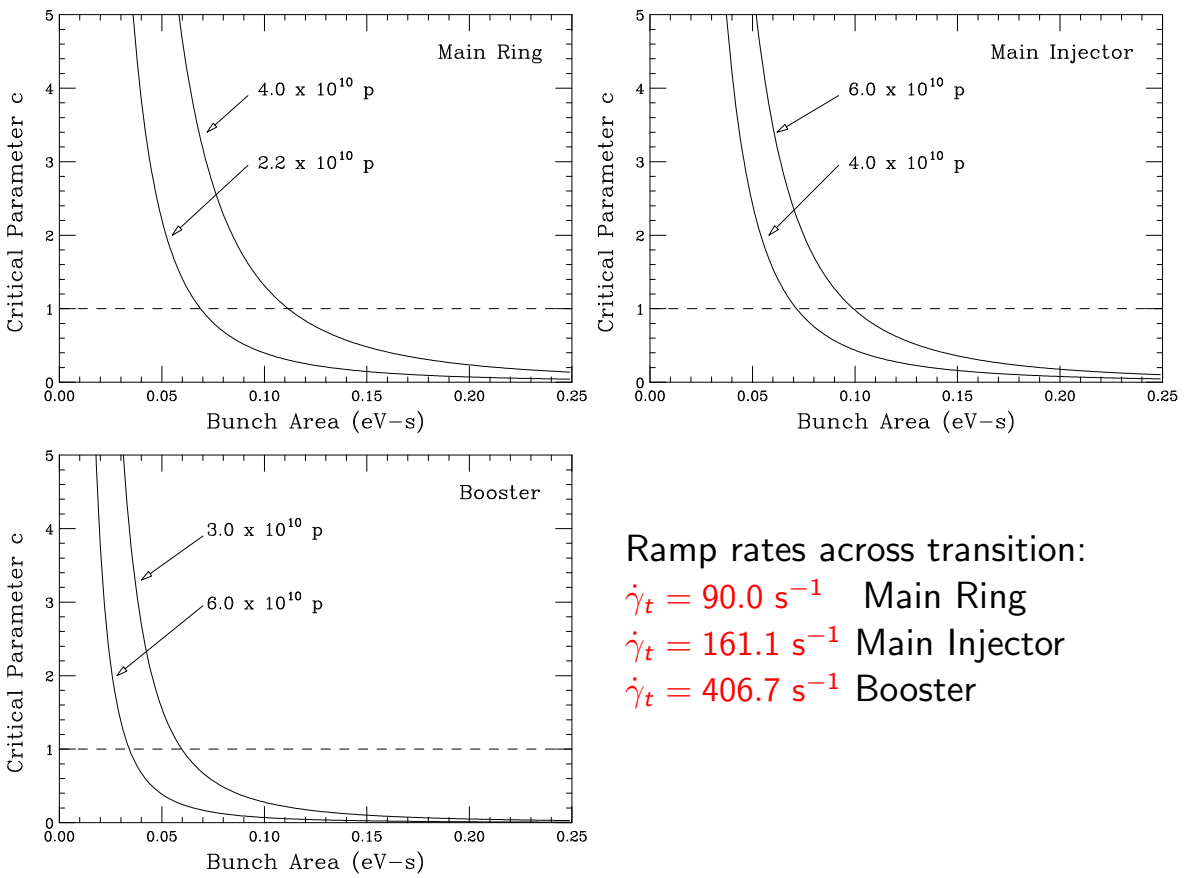

Ramp rates across transition: $\dot{\gamma}_{t}=90.0 \mathrm{~s}^{-1} \quad$ Main Ring $\dot{\gamma}_{t}=161.1 \mathrm{~s}^{-1}$ Main Injector $\dot{\gamma}_{t}=406.7 \mathrm{~s}^{-1}$ Booster 


\section{Growths at Cutoff and High Frequencies}

- At cutoff freq., growth rate small but seeds from beam are large. At high freq., growth rate huge but seeds from Schottky noise tiny.

- So which one gives higher final growth in amplitude or power.

- We analyze this problem analytically.

- Results are given for the old Fermilab Main Ring.

$\dot{\gamma}_{t} \quad N_{b}$ Initial Bunch Emittance Final Power Spectrum of Fluctuation

\begin{tabular}{cccccc}
$\left(\mathrm{s}^{-1}\right)$ & $\left(10^{10}\right)$ & $(\mathrm{eV}-\mathrm{s})$ & at $n_{\text {cutoff }}$ & at $n_{\max }$ & sum \\
\hline 90 & 2.2 & 0.05 & 3.70 & $1.50 \times 10^{9}$ & $4.03 \times 10^{10}$ \\
90 & 2.2 & 0.06 & 2.21 & $1.08 \times 10^{2}$ & $3.97 \times 10^{3}$ \\
90 & 2.2 & 0.07 & 1.67 & $1.19 \times 10^{-2}$ & $5.74 \times 10^{-1}$ \\
90 & 2.2 & 0.08 & 1.41 & $4.86 \times 10^{-5}$ & $2.93 \times 10^{-3}$ \\
90 & 2.2 & 0.09 & 1.26 & $1.41 \times 10^{-6}$ & $1.06 \times 10^{-4}$ \\
\hline 120 & 4.0 & 0.06 & 7.44 & $4.37 \times 10^{18}$ & $1.00 \times 10^{20}$ \\
120 & 4.0 & 0.07 & 3.80 & $1.94 \times 10^{9}$ & $5.83 \times 10^{10}$ \\
120 & 4.0 & 0.08 & 2.54 & $4.40 \times 10^{3}$ & $1.67 \times 10^{5}$ \\
120 & 4.0 & 0.09 & 1.95 & $1.02 \times 10^{0}$ & $4.76 \times 10^{1}$ \\
120 & 4.0 & 0.10 & 1.64 & $3.57 \times 10^{-3}$ & $2.00 \times 10^{-1}$
\end{tabular}




\section{Difficulties in Simulation}

- For Fermilab Main Ring, half-value space-charge roll-off harmonic $n_{\frac{1}{2}}$ corresponds to $134 \mathrm{GHz}$.

- In simulations need bin size $1 /(2 \times 134)=0.00373$ ns.

- RF wavelength or bucket width $18.8 \mathrm{~ns} \longrightarrow$ need at least 4096 bins.

- Simulation results across transition using ESME. [26]
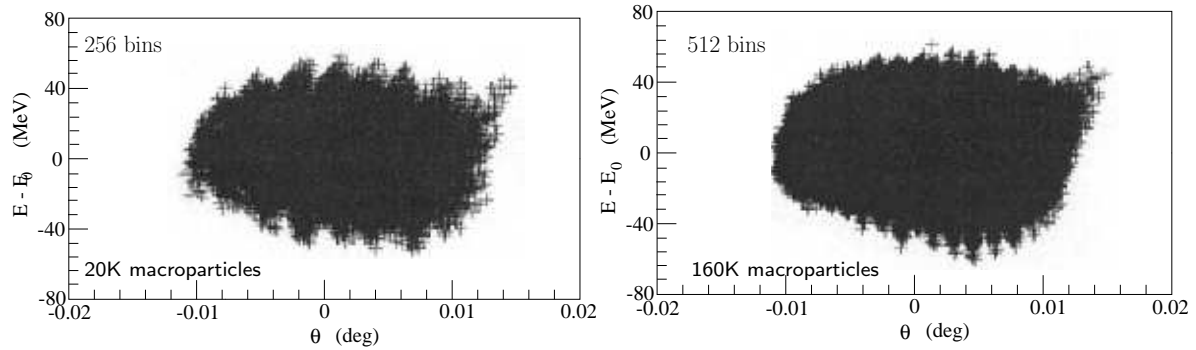

- Because space charge force $\propto$ gradient of distribution. To maintain same accuracy, 3-in-1 rule says that macroparticle number must increase by $2^{3}$ when bin width is reduced by a factor of 2. [27]

- Thus tracking time will increase by a factor of $2^{4}$. 


\section{Right Amount of Schottky Noise}

- In simulation of microwave instability, there is usually ample time for instability to develop to saturation. Therefore, we do not care so much about the size of the initial excitation or seed of the growth.

- Across transition, however, the bunch is negative-mass unstable only for a short time until the slip factor $\eta$ becomes large enough to provide enough Landau damping.

This time is typically of the order of the nonadiabatic time, about $3 \mathrm{~ms}$ for the Fermilab Main Ring. Therefore right amount of seeds is very important.

- Relative size of Schottky noise $\propto \frac{1}{\sqrt{N_{M}}}$, where $N_{M}$ is no. of macroparticles.

- But the right amount of Schottky noise is $\sim \frac{1}{\sqrt{N_{b}}}$, where $N_{b} \sim 2 \times 10^{10}$.

- Since it is impossible to use so many particles in simulation, most reported simulations across transition are incorrect. 


\section{Low-Discrepancy Sequence [28]}

- Since we must use macroparticles of number much less than number of the microparticles, Schottky noise is very much larger.

- But if we populate the macroparticles in more orderly way, Schottky noise can be reduced.

- This way of population is according to a low-discrepancy sequence.

- Population of 50 particles randomly (left) and according to Hammersley sequence (right).
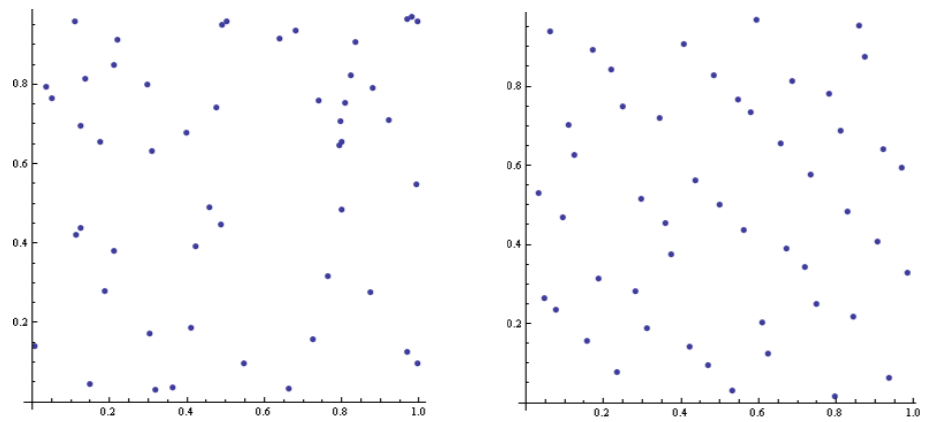

- Fluctuation of $N$ particle becomes $\mathcal{O}(1)$ instead of $\mathcal{O}(\sqrt{N})$. 


\section{Population of 10,000 particles:}

random

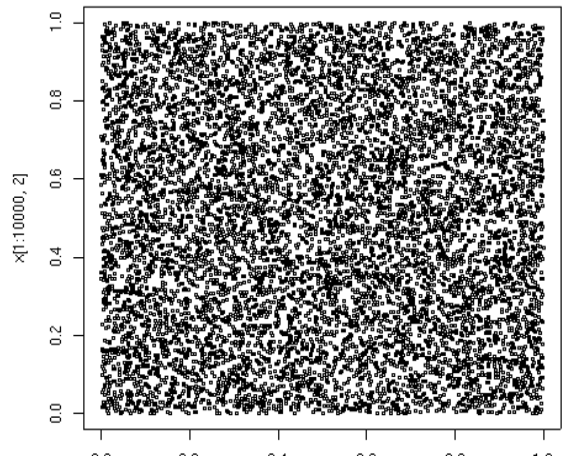

low-discrepancy

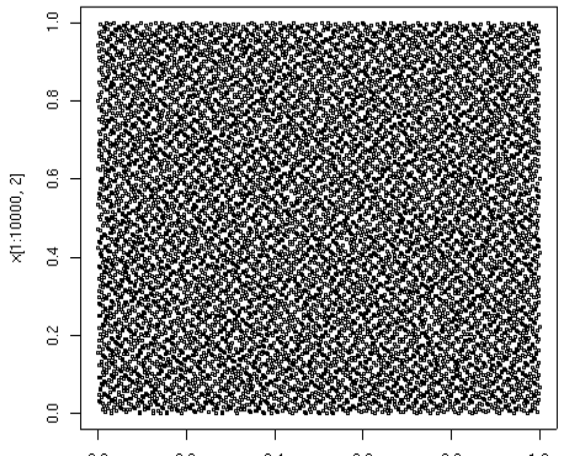

Relative noise level:

$$
\frac{\sqrt{N}}{N}=3.2 \times 10^{-3}
$$

$$
\frac{1}{N}=1 \times 10^{-5}
$$




\section{Improvement with Hammersley Sequence}

\begin{tabular}{|c|c|c|}
\hline & With Microparticles & $\begin{array}{c}\text { With Macroparticles } \\
\text { Hammersley Sequence }\end{array}$ \\
\hline $\begin{array}{c}\text { average no. } \\
\text { per bin }\end{array}$ & $\Delta N=\frac{N_{b}}{M}$ & $\Delta N_{M}=\frac{N_{M}}{M}$ \\
\hline $\begin{array}{c}\text { fluctuation } \\
\text { function }\end{array}$ & $f\left(\Delta \phi_{n}\right)=\frac{\delta N_{n}}{\Delta N}$ & $f\left(\Delta \phi_{n}\right)=\frac{\delta N_{n}}{\Delta N_{M}}$ \\
\hline $\begin{array}{c}\text { Expectation } \\
\text { of errors }\end{array}$ & $E\left(\delta N_{n} \delta N_{m}\right)=\delta_{n m} \Delta N F\left(\Delta \phi_{n}\right)$ & $E\left(\delta N_{n} \delta N_{m}\right)=\delta_{n m}$ \\
\hline & $E\left(\left|c_{k_{b}}(0)\right|^{2}\right)=\frac{1}{N_{b}}$ & $E\left(\left|c_{k_{b}}(0)\right|^{2}\right)=\frac{1}{M\left(\Delta N_{M}\right)^{2}}=\frac{M}{N_{M}^{2}}$ \\
\hline
\end{tabular}

- $N_{b}$ is no. of microparticles, $N_{M}$ is no. of macroparticles

- Thus number of macroparticles required for the same Schottky noise is $N_{M}=\left(M N_{b}\right)^{\frac{1}{2}}$

For Fermilab MR, $N_{M} \sim 2.4$ to $3.6 \times 10^{6}$, instead of $N_{b}=2.2 \times 10^{10}$ 


\section{Two Difficulties}

- Formerly $E\left[f^{2}\left(\Delta \phi_{m}, 0\right)\right]=E\left[\frac{\delta N_{m}^{2}}{\Delta N^{2}}\right]=\frac{F\left(\Delta \phi_{m}\right)}{\Delta N}=\frac{M}{N_{b}} F\left(\Delta \phi_{m}\right)$

- Now $E\left[f^{2}\left(\Delta \phi_{m}, 0\right)\right]=\frac{1}{\Delta N_{M}^{2}}=\frac{M^{2}}{N_{M}^{2}}$ which is not proportional to $F\left(\Delta \phi_{m}\right)$. Difficulty 1: Thus relative fluctuations in bins have changed.

- Difficulty 2: Fluctuation of particles may change in time.

- Because of space charge, rf bucket will be modified.

- Usual way of population is to populate as if no space charge.

Then turn on space charge and let particles fit the rf bucket after a number of synchrotron oscillations.

- But synchrotron tune is amplitude dependent.

- Try simulation of $2 \times 10^{5}$ particles in 20 bins of equal size. Assume syn. tune to decrease linearly by $1 \%$ from bunch's center to edge. 
- Distribution is projected onto time or phase axis.

- Number of particles in excess of a smooth Gaussian is recorded for each bin

- Result: rms fluctuation starts from 7 , increases rapidly to 20 after 20 turns, and 50 after 100 turns.

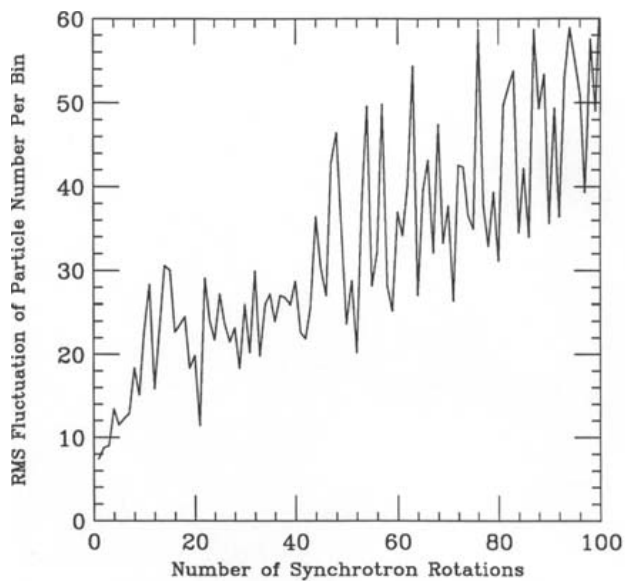

- Thus the advantage of a smaller noise level by using a Hammersley sequence can be lost rapidly when particles are shuffled.

Care must be taken and many tests have to be made when such population is used. 


\section{Undulator Radiation $[29,30,31]$}

- Consider a planar undulator with sinusoidal magnetic field in $y$-direction.

Suppose undulator period is $\lambda_{u}$, magnetic field is

$B_{y}(z)=B_{0} \sin k_{u} z$

$B_{x}=B_{z}=0 \quad$ with $\quad k_{u}=\frac{2 \pi}{\lambda_{u}}$

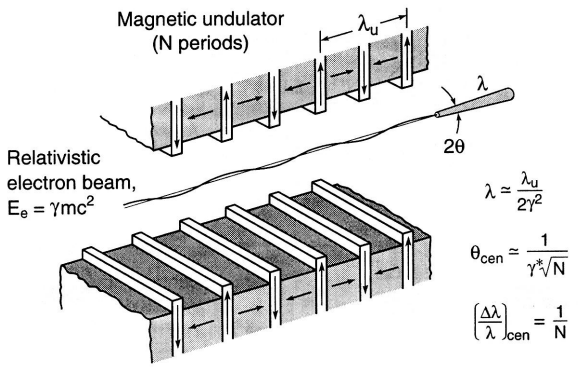

- Electron motion $m \gamma \frac{d^{2} x}{d t^{2}}=e\left(v_{y} B_{z}-v_{z} B_{y}\right)=-e B_{0} c \sin k_{u} z$

- Changing $t$ to $z$ and assume $v_{z} \approx c, x^{\prime \prime}=-\frac{e B_{0}}{\gamma m c} \sin k_{u} z$

- Since $\gamma$ is constant in a static magnetic field,

$x^{\prime}=\frac{e B_{0}}{\gamma m c k_{u}} \cos k_{u} z \equiv \frac{K}{\gamma} \cos k_{u} z, \quad x=\frac{K}{\gamma k_{u}} \sin k_{u} z$

where $K=\frac{e B_{0}}{m c k_{u}}=0.9337 B_{0}[$ Tesla $] \lambda_{u}[\mathrm{~cm}], \quad \frac{K}{\gamma} \sim$ deflection angle is the dimensionless deflection parameter or undulator parameter 


\section{Undulator Radiation}

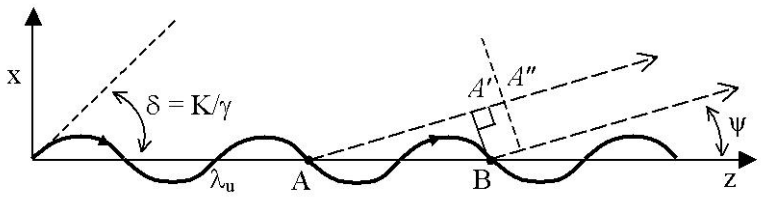

- Electron moves along arc length $\widetilde{A B}$, radiation along straight line $\overline{A A^{\prime}}$. Their time difference is one radiation wavelength $\lambda_{1} / c$.

- $\frac{\lambda_{1}}{c}=\frac{\widetilde{A B}}{v}-\frac{\overline{A A^{\prime}}}{c}$

with $\widetilde{A B}=\int_{0}^{\lambda_{u}} d z \sqrt{1+x^{\prime 2}} \approx \int_{0}^{\lambda_{u}} d z\left[1+\frac{K^{2}}{2 \gamma^{2}} \cos ^{2} k_{u} z\right]=\lambda_{u}\left(1+\frac{K^{2}}{4 \gamma^{2}}\right)$

and $\overline{A A^{\prime}}=\lambda_{u} \cos \psi \approx \lambda_{u}\left(1-\frac{\psi^{2}}{2}\right)$

- Get $\frac{\lambda_{1}(\psi)}{\lambda_{u}}=\frac{1+K^{2} / 4 \gamma^{2}}{\beta}-\left(1-\frac{\psi^{2}}{2}\right) \approx \frac{1+K^{2} / 2+\gamma^{2} \psi^{2}}{2 \gamma^{2}}$ 
- Example $\lambda_{u}=5 \mathrm{~cm}, B_{0}=0.35 \mathrm{~T}, K=0.934 \times 0.35 \times 5=1.6$

- At $E=5 \mathrm{GeV}, \lambda_{1}(0)=5.7 \AA$

- For one electron, emission at one undulator magnet is in phase with emission at the next magnet. Thus for $N$ undulator periods, emission adds up in phase.
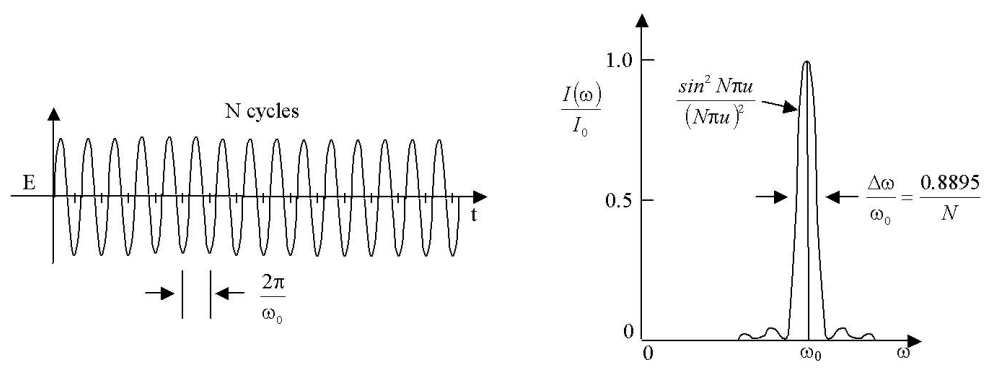

- However, emission from one electron usually is not in phase from that of other electrons, unless they are within $\lambda_{1} / 2$.

- Thus of beam is microbunched in wavelength of $\lambda_{1} / 2$, electrons within each bunchlet will emit in phase, or emission will be coherent. 
- Emission freq depends on $\gamma$. which has a spread in beam.

- Thus there is a spread in $\lambda_{1}$.

- In presence of a laser beam of wavelength $\lambda_{1}$, electrons will be driven into synchrotron oscillation in buckets setup by $E$ field of laser.

- This pendulum motion is first discovered by Colson. [32]
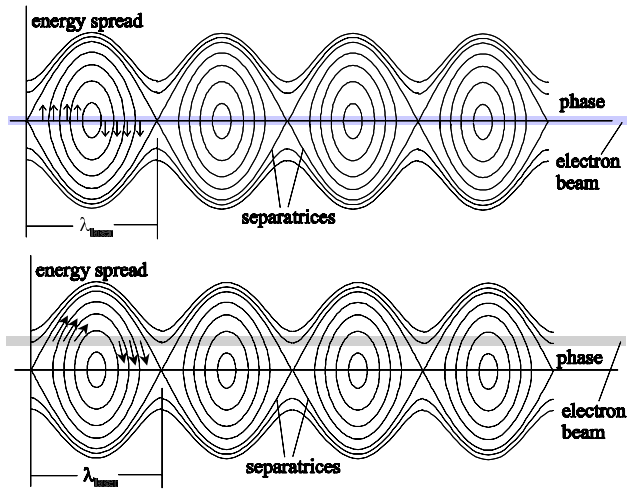

- Electron interacts with $E$ of laser with power gain $\vec{E} \cdot \vec{v}$.

- This has to be a loss in order intensity of laser is increases.

- This can be accomplished to offsetting initial energy of electron beam.

- In $\frac{1}{4}$ of syn. period, beam will point in $\gamma$-direction and narrow in $z$-direction. Emission mostly coherent and power is peaked. 


\section{Types of FEL}

1. Mirrors at right position so that reflected emission wave is in phase with undulator.

Good for low freq only, because there are no high freq mirrors.

2. There is an input laser pulse that induces stimulus emission at undulator, and microbunches electron beam.

3. Electron beam is microbunched by emission wave - called SASE.

FEL Oscillator

Light Envelope

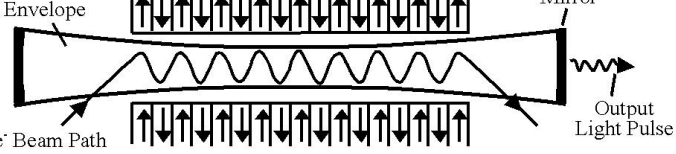

UNDULATOR

NOTE: Requires Mirrors

Seeded (Amplifier) FEL

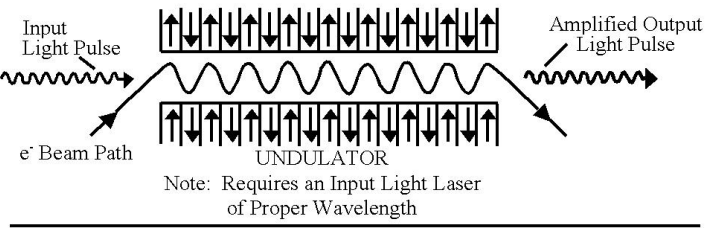

Self-Amplified-Spontaneous-Emission (SASE) FEL

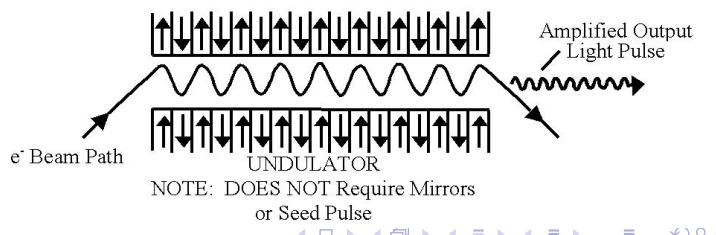




\section{Low-Energy Electron Storage Ring at ICEEM}

- Using the 4 dipoles left behind from the cooler injector (CIS) at ICEEM, a low energy electron storage ring has been built.

- Ring circumference $20 \mathrm{~m}$, energy 50-200 MeV

- Dipoles: $\rho=1.273 \mathrm{~m}, L=2 \mathrm{~m}$, edge angle $12^{\circ}$.

- $\mathcal{J}_{x}<0 \Longrightarrow$ horizontal motion unstable.

- $\mathcal{J}_{x}$ can be adjusted by two Robinson wigglers.

- Dispersion can be changed in main dipoles, so momentum compaction $\alpha$ is tunable.

- want to produce coherent $1 \mathrm{THz} X$-ray in the ring.

- Need micro-bunching of beam to 1 ps or $\lambda \sim 300 \mu \mathrm{m}$.

- Use impedance to create controlled microwave-like instability and microbunching. 


\section{Diffraction Grating}

- Beam through cavity-like structure generates wake.

- $E_{z}$ of $\mathrm{TM}_{0 n p}$ modes provides controllable microbunching for the required frequency.

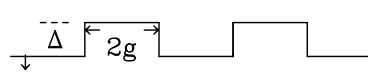

$2 \mathrm{~b}$

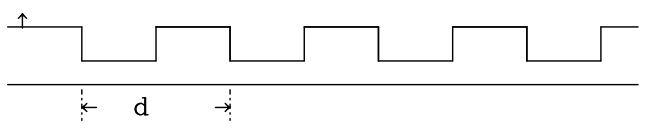

- $Z_{\|}(\omega)=\frac{g Z_{0}}{\pi b I_{0}^{2}\left(\frac{k b}{\beta \gamma}\right) D}$

$D=j \frac{R_{0}^{\prime}(k b)}{R_{0}(k b)}-2 j k\left[\sum_{s=1}^{s} \frac{1}{\beta_{s}^{2} b}\left(1-e^{-j \beta_{s} g} \frac{\sin \beta_{s} g}{\beta_{s} g}\right)\right.$

$$
\left.-\sum_{s=S+1}^{\infty} \frac{1}{\alpha_{s}^{2} b}\left(1-e^{-\alpha_{s} g} \frac{\sinh \alpha_{s} g}{\alpha_{s} g}\right)\right]
$$

- $k=\omega / c$

$R_{0}(k b)=J_{0}(k b) Y_{0}(k a)-J_{0}(k a) Y_{0}(k b)$, with $a=b+\Delta$

$\beta_{s} b=\sqrt{k^{2} b^{2}-j_{0 s}^{2}}$ and $\alpha_{s} b=\sqrt{j_{0 s}^{2}-k^{2} b^{2}}$,

where $j_{0 s}$ is sth zero of $J_{0}$ and $j_{0 s}$ is zero just larger than or equal to $k b$. 


\section{$I_{0}$ Factor}

- $I_{0}^{2}\left(\frac{k b}{\beta \gamma}\right)$ occurs in the denominator.

- Image of a point charge on beam pipe has rms length $\sigma_{\tau}=\frac{b}{\sqrt{2} \beta \gamma}$.

- The Fourier spectrum of image current is $\frac{1}{I_{0}\left(\frac{k b}{\beta \gamma}\right)}$.

- $\frac{1}{I_{0}^{2}\left(\frac{k b}{\beta \gamma}\right)}$ can become very small at high frequency and low energy.

- This is the only particle-energy dependent factor in the impedance.

- It constitutes a measure of the efficiency at which the beam particle generates electromagnetic fields and excites a resonance in the cavity.

- Energy is low at the Indiana ring.

This limits the highest frequency $X$-ray generated to $\lesssim 1 \mathrm{THz}$ 


\section{Illustration of Impedance}
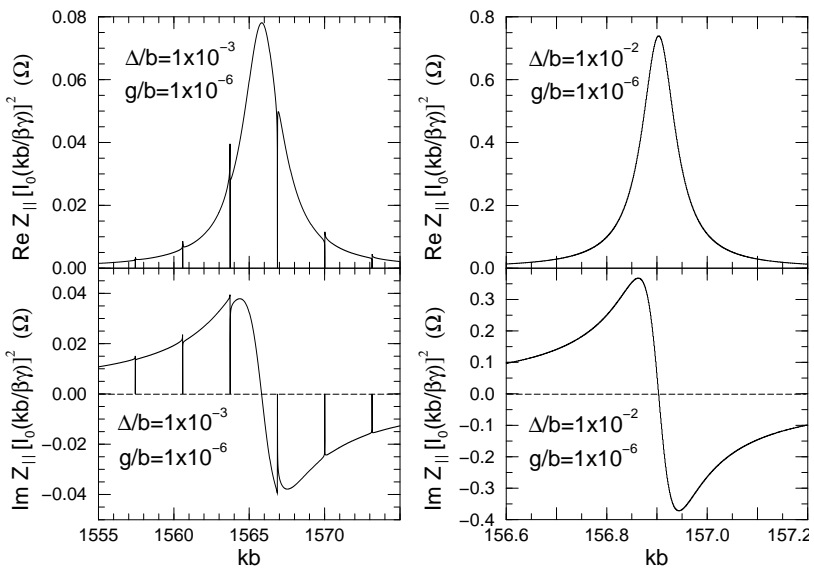

For $1 \mathrm{THz}, b=4 \mathrm{~cm}$ $k b=838$ $\Delta=75 \mu \mathrm{m}$ $\frac{\Delta}{b}=1.88 \times 10^{-3}$

- Center freq of 1 st broad-band of 1 cavity is at $k \Delta \approx \frac{\pi}{2}$ when $\frac{g}{b} \rightarrow 0$.

- It is shifted slightly downward as $\frac{g}{b}$ increases and $\frac{\Delta}{b}$ decreases.

- The center freq. is approx. $f_{c} \approx \frac{C}{4 \Delta}$. 


\section{Quality Factor and Impedance}

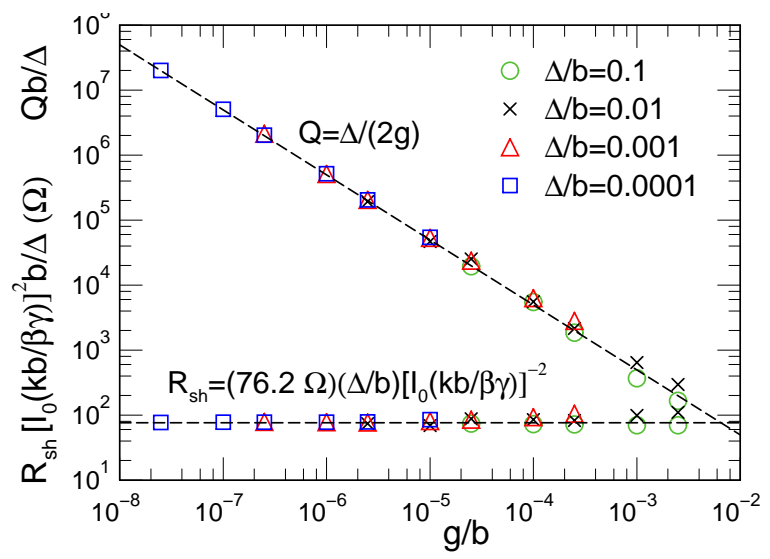

- Quality factor ( $Q$-value) of resonance generated by the grating structure depends essentially on $\Delta / g$

- Shunt impedance depends essentially on $\Delta / b$.

- Impedance becomes $\left|\frac{Z_{\|}}{n}\right| \approx \frac{300 \beta \Delta^{2}}{2 \pi R b} \Omega$.

$\left[I_{0}^{2}\right.$ factor not included $]$ 


\section{Microwave Instabilities}

- With $N$ cavities, diffraction phenomenon takes place by enhancing some resonances $N$-fold and shrinking width $N$-fold within the envelope of broadband resonance of a single cavity.

- This impedance is used to generate microbunching.

- Condition of controlled microwave instability (Keil-Schnell):

$e l_{\mathrm{pk}} \beta^{2}\left|\frac{Z_{\|}}{n}\right| \geq 2 \pi E \sigma_{\delta}^{2}|\eta| F_{\text {dist }} \approx 1.23 \times 10^{-6} \frac{\gamma^{3}|\eta|}{\mathcal{J}_{E} \rho[\mathrm{m}]}$

$I_{\mathrm{pk}}=F_{\mathrm{B}} I_{\mathrm{av}}$ is the peak current,

$l_{\mathrm{av}}$ is the average current,

$F_{\mathrm{B}}=2 \pi R / \sqrt{2 \pi} \sigma_{z}$ is the bunching factor,

$\mathcal{J}_{E} \approx 2$ is the longitudinal damping partition number,

$\sigma_{\delta}=\sqrt{\frac{3.83 \times 10^{-13} \gamma^{2}}{\rho \mathcal{J}_{E}}}$ is natural momentum spread. 


\section{Application to ICEEM Ring}

- Consider generation of $f_{\mathrm{coh}}=1 \mathrm{THz}$ radiation.

- Minimum electron energy required is $\gamma=\left(4 \pi f_{\operatorname{coh}} \rho / 3 c\right)^{1 / 3} \geq 26$.

- Consider 100-Mev electrons $(\gamma=196)$.

Get êे $\beta^{2}\left|\frac{Z_{\|}}{n}\right| \geq 3.37|\eta| \mathrm{eV}$.

- If $I_{\mathrm{pk}}=1 \mathrm{kA}$, requires $\left|\frac{Z_{\|}}{n}\right| \geq 3.37 \times 10^{-3}|\eta| \Omega$.

- $1 \mathrm{THz}$ radiation $\Longrightarrow$ grating depth $\Delta \approx 75 \mu \mathrm{m}$ and rev. harmonic $n=6.7 \times 10^{4}$.

- Structure radius $b=4 \mathrm{~cm}$ gives $\left|\frac{Z_{\|}}{n}\right|=1.02 \times 10^{-8} \Omega$

$\longrightarrow N=10000$ gratings required if $|\eta| \lesssim 0.003$. 


\section{Maximum Slip Factor Required}

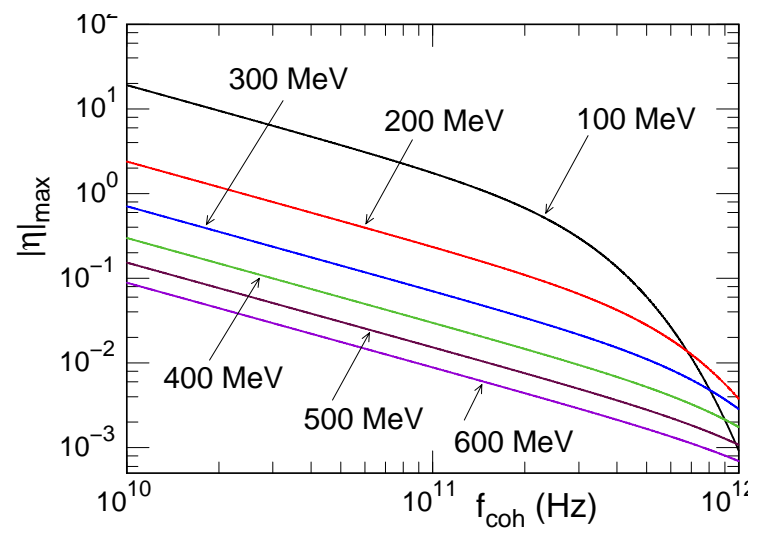

- For each coherent radiation frequency $f_{\text {coh }}$, plot gives maximum $|\eta|$ required for Keil-Schell instability or microbunching.

- We use grating depth $\Delta=c /\left(4 f_{\text {coh }}\right)$,

$g=\Delta$ (groove half width equal depth), grating period $d=4 g$, total length of gratings $1 \mathrm{~m}$, peak current $I_{\mathrm{pk}}=100 \mathrm{~A}$. 


\section{Simulation}

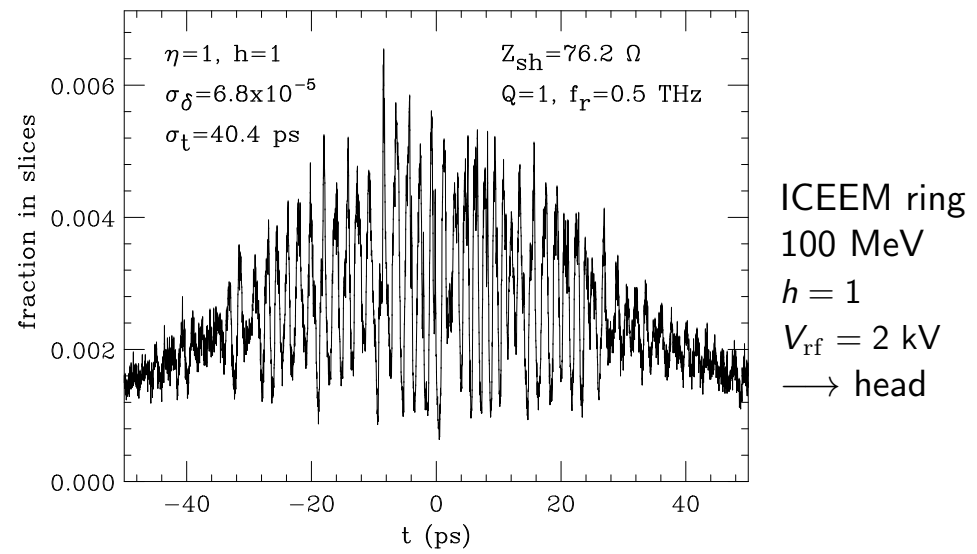

- Bin width 0.04 ps, 80,000 macroparticles used.

- Keil-Schnell limit of stability is $I_{\mathrm{pk}}=12.7 \mathrm{~A}$.

- However, $I_{\mathrm{pk}}=90 \mathrm{~A}$ was used so that microbunching develops fast before peaks are overlapped. 


\section{Higher-Frequency Coherent Radiation}

- The grating method sets limit to coherent radiation freq. in ICEEM ring.

There are 2 reasons:

(1) Since the ring can reach $200 \mathrm{Mev}$ only, the $\frac{1}{I_{0}^{2}(k b / \beta \gamma)}$ factor becomes too small when $\gtrsim 1 \mathrm{THz}$.

(2) The grating depth cannot be made too shallow technically.

- To achieve higher frequency coherent radiation, need another method.

- The method of inverse Compton scattering can be used. 


\section{Inverse Compton Scattering}

- Compton scattering is for photon scattered by electron in rest frame.

- Now electron in the beam travels at high speed.

- Inverse Compton scattering is for a photon collide with the electron head-on and its direction is reverse,

- There is one Lorentz transformation to bring incident photon to electron rest frame.

- There is another Lorentz transformation to boost the direction-reverse photon back to the lab frame.

- Two Lorentz transformations increases photon freq. by the factor $\sim 4 \gamma^{2}$.

- If we start from a laser beam of freq $f_{L}=3 \times 10^{14} \mathrm{~Hz}(1000 \AA)$, the back-scattered photon has frequency $f_{s}=4 \gamma^{2} f_{L}=1.15 \times 10^{19} \mathrm{~Hz}$ with $\gamma=196$ or $100-\mathrm{MeV}$ electrons. $\left(\lambda_{s}=8.70 \times 10^{-20} \mathrm{~s}\right.$. $)$ 


\section{Undulator Analog}

- The electron traveling towards the head-on laser beam sees alternating horizontal $\mathrm{E}$ field and alternating vertical $\mathrm{B}$ field.

- Both fields steer the electron in an oscillatory path in horizontal plane.

- the effect of $E$ and $B$ fields add, because electron and photon are in opposite directions.

- This resembles the electron traversing an undulator, with undulator wavelength $\lambda_{u} \approx \frac{\lambda_{L}}{2}$.

- It can be shown that the undulator concept gives relatively the same intensity of emitted photon per electron per unit angular frequency and per unit solid angle, $\frac{d^{2} N}{d \omega d \Omega}$. 


\section{Microbunching}

- The electron bunch passes through an undulator, it will be micro-bunched to the forward radiating photon wavelength.

- Now the electron bunch passes through the incident laser beam will also be micro-bunched to the inverse-scattered photon wavelength.

- Photons scattered by each micro-bunched slice of the electron bunch will be coherent.

- It takes time for the intensity of scattered photon to grow, the growth length $L_{g}=\frac{\lambda_{u}}{4 \pi \sqrt{3} \rho}$, and to saturate, the saturation length $L_{\text {sat }}=\frac{\lambda_{u}}{\rho} . \quad$ ( $\rho$ is Pierce parameter)

- We need to make sure that during this time, the electron micro-bunched slice will not drift by more than one wavelength of the scattered photon or $\lambda_{s}=8.70 \times 10^{-20} \mathrm{~s}$.

- This means that the slip factor of the ring $\eta$ must be kept very small. 


\section{FEL or Pierce Parameter [34]}

- The Pierce parameter is an important parameter in FEL.

- It is dimensionless and is defined as $\rho=\left[\frac{r_{e} \lambda_{u}^{2} K^{2}[J J]^{2} n_{e}}{32 \pi \gamma^{3}}\right]^{1 / 3}$

$r_{e}$ is electron classical radius,

$$
\begin{aligned}
& {\left[J J=J_{0}(\xi)-J_{1}(\xi), \quad \xi=\frac{K^{2}}{4+2 K^{2}}=\frac{1}{2\left(1+2 / K^{2}\right)}<\frac{1}{2} .\right.} \\
& n_{e}=\frac{N_{B}}{\sqrt{2 \pi} \sigma_{\tau} c \sqrt{2 \pi} \sigma_{x} \sqrt{2 \pi} \sigma_{y}}=\frac{I_{p} / e}{2 \pi c \sigma_{x} \sigma_{y}} \text { is peak electron density, }
\end{aligned}
$$

$I_{p}$ is peak current, and $\sigma_{x}$ and $\sigma_{y}$ are rms sizes of electron beam.

- 1. $\rho=\frac{\text { field energy generated }}{\text { e-beam kinetic energy }}, \therefore$ saturated power $\sim \rho \times$ e-beam power

2. Saturated length $L_{\text {sat }} \sim \frac{\lambda_{u}}{\rho}$.

3. Final saturated energy spread of e-beam $\sim \rho$.

4. Transverse size of laser beam $\sigma_{r} \sim \sqrt{\frac{\lambda_{1}}{4 \pi} \frac{\lambda_{u}}{4 \pi \rho}}$. 


\begin{tabular}{|c|c|c|c|c|c|c|c|c|c|c|}
\hline$\sigma_{x}(\mathrm{~m})$ & $\sigma_{z}(\mathrm{~m})$ & $\sigma_{t}(\mathrm{~s})$ & $\sigma_{s}(\mathrm{~m})$ & $\lambda_{u}(\mathrm{~m})$ & $\begin{array}{l}q \\
\text { (coulumb) }\end{array}$ & $N_{0}\left(\mathrm{~m}^{3}\right)$ & fact & $E_{r}(\mathrm{eV})$ & $\gamma_{r}$ & $\epsilon$ \\
\hline \multirow[t]{2}{*}{$6.32 \mathrm{E}-05$} & $6.32 \mathrm{E}-05$ & $1.00 \mathrm{E}-11$ & $3.00 \mathrm{E}-03$ & $1.00 \mathrm{E}-06$ & $1.00 \mathrm{E}-08$ & $1.66 \mathrm{E}+21$ & $4.48 \mathrm{E}-16$ & $2.50 \mathrm{E}+07$ & 48.92 & $8.00 \mathrm{E}-08$ \\
\hline & $1.26 \mathrm{E}-08$ & $\leftarrow \operatorname{area}\left(\mathrm{m}^{2}\right)$ & ) $3.00 \mathrm{E}+14$ & $\leftarrow \mathrm{Hz}$ & & & & & & $3.91 \mathrm{E}-06$ \\
\hline$C(\mathrm{~m})$ & $T_{0}(\mathrm{~s})$ & $f_{0}(\mathrm{~Hz})$ & $\rho_{0}(\mathrm{~m})$ & $\delta_{0}$ & & & & & & \\
\hline 20 & $6.67 \mathrm{E}-08$ & $1.50 \mathrm{E}+07$ & 1.273 & $1.898 \mathrm{E}-05$ & & & & & & \\
\hline $\begin{array}{l}P \\
(\mathrm{~W})\end{array}$ & $\begin{array}{l}I \\
\left(\mathrm{~W} / \mathrm{m}^{2}\right)\end{array}$ & $\begin{array}{l}u \\
\left(\mathrm{~J} / \mathrm{m}^{3}\right)\end{array}$ & $\begin{array}{l}E \\
(\mathrm{~V} / \mathrm{m})\end{array}$ & $\begin{array}{l}B \\
(\mathrm{~T})\end{array}$ & $K_{w}$ & $\rho$ & $\begin{array}{l}L_{G} \\
(\mathrm{~m})\end{array}$ & $\begin{array}{l}L_{\text {sat }} \\
(\mathrm{m})\end{array}$ & $N_{\text {path }}$ & $|\eta|$ \\
\hline $1.00 \mathrm{E}+03$ & $7.96 \mathrm{E}+10$ & $2.65 \mathrm{E}+02$ & $5.48 \mathrm{E}+06$ & $1.83 \mathrm{E}-02$ & $1.71 \mathrm{E}-06$ & $1.05 \mathrm{E}-08$ & $4.38 \mathrm{E}+00$ & $8.76 \mathrm{E}+01$ & 29213 & $9.42 \mathrm{E}-12$ \\
\hline $3.16 \mathrm{E}+03$ & $2.52 \mathrm{E}+11$ & $8.39 \mathrm{E}+02$ & $9.74 \mathrm{E}+06$ & $3.25 \mathrm{E}-02$ & $3.03 \mathrm{E}-06$ & $3.88 \mathrm{E}-08$ & $1.18 \mathrm{E}+00$ & $2.37 \mathrm{E}+01$ & 7897 & $3.48 \mathrm{E}-11$ \\
\hline $1.00 \mathrm{E}+04$ & $7.96 \mathrm{E}+11$ & $2.65 \mathrm{E}+03$ & $1.73 \mathrm{E}+07$ & $5.78 \mathrm{E}-02$ & $5.39 \mathrm{E}-06$ & $5.69 \mathrm{E}-08$ & $8.07 \mathrm{E}-01$ & $1.61 \mathrm{E}+01$ & 5380 & $5.11 \mathrm{E}-11$ \\
\hline $3.16 \mathrm{E}+04$ & $2.52 \mathrm{E}+12$ & $8.39 \mathrm{E}+03$ & $3.08 \mathrm{E}+07$ & $1.03 \mathrm{E}-01$ & $9.59 \mathrm{E}-06$ & $8.36 \mathrm{E}-08$ & $5.50 \mathrm{E}-01$ & $1.10 \mathrm{E}+01$ & 3666 & $7.51 \mathrm{E}-11$ \\
\hline $1.00 \mathrm{E}+05$ & $7.96 \mathrm{E}+12$ & $2.65 \mathrm{E}+04$ & $5.48 \mathrm{E}+07$ & $1.83 \mathrm{E}-01$ & $1.71 \mathrm{E}-05$ & $1.23 \mathrm{E}-07$ & $3.75 \mathrm{E}-01$ & $7.49 \mathrm{E}+00$ & 2497 & $1.10 \mathrm{E}-10$ \\
\hline $3.16 \mathrm{E}+05$ & $2.52 \mathrm{E}+13$ & $8.39 \mathrm{E}+04$ & $9.74 \mathrm{E}+07$ & $3.25 \mathrm{E}-01$ & $3.03 \mathrm{E}-05$ & $1.80 \mathrm{E}-07$ & $2.55 \mathrm{E}-01$ & $5.10 \mathrm{E}+00$ & 1701 & $1.62 \mathrm{E}-10$ \\
\hline $1.00 \mathrm{E}+06$ & $7.96 \mathrm{E}+13$ & $2.65 \mathrm{E}+05$ & $1.73 \mathrm{E}+08$ & $5.78 \mathrm{E}-01$ & $5.39 \mathrm{E}-05$ & $2.64 \mathrm{E}-07$ & $1.74 \mathrm{E}-01$ & $3.48 \mathrm{E}+00$ & 1159 & $2.37 \mathrm{E}-10$ \\
\hline $3.16 \mathrm{E}+06$ & $2.52 \mathrm{E}+14$ & $8.39 \mathrm{E}+05$ & $3.08 \mathrm{E}+08$ & $1.03 \mathrm{E}+00$ & $9.59 \mathrm{E}-05$ & $3.88 \mathrm{E}-07$ & $1.18 \mathrm{E}-01$ & $2.37 \mathrm{E}+00$ & 790 & $3.48 \mathrm{E}-10$ \\
\hline $1.00 \mathrm{E}+07$ & $7.96 \mathrm{E}+14$ & $2.65 \mathrm{E}+06$ & $5.48 \mathrm{E}+08$ & $1.83 \mathrm{E}+00$ & $1.71 \mathrm{E}-04$ & $5.69 \mathrm{E}-07$ & 8.07E-02 & $1.61 \mathrm{E}+00$ & 538 & $5.11 \mathrm{E}-10$ \\
\hline $3.16 \mathrm{E}+07$ & $2.52 \mathrm{E}+15$ & $8.39 \mathrm{E}+06$ & $9.74 \mathrm{E}+08$ & $3.25 \mathrm{E}+00$ & $3.03 \mathrm{E}-04$ & $8.36 \mathrm{E}-07$ & $5.50 \mathrm{E}-02$ & $1.10 \mathrm{E}+00$ & 367 & $7.51 \mathrm{E}-10$ \\
\hline $1.00 \mathrm{E}+08$ & $7.96 \mathrm{E}+15$ & $2.65 \mathrm{E}+07$ & $1.73 \mathrm{E}+09$ & $5.78 \mathrm{E}+00$ & $5.39 \mathrm{E}-04$ & $1.23 \mathrm{E}-06$ & $3.75 \mathrm{E}-02$ & $7.49 \mathrm{E}-01$ & 250 & $1.10 \mathrm{E}-09$ \\
\hline $3.16 \mathrm{E}+08$ & $2.52 \mathrm{E}+16$ & $8.39 \mathrm{E}+07$ & $3.08 \mathrm{E}+09$ & $1.03 \mathrm{E}+01$ & $9.59 \mathrm{E}-04$ & $1.80 \mathrm{E}-06$ & $2.55 \mathrm{E}-02$ & $5.10 \mathrm{E}-01$ & 170 & $1.62 \mathrm{E}-09$ \\
\hline $1.00 \mathrm{E}+09$ & $7.96 \mathrm{E}+16$ & $2.65 \mathrm{E}+08$ & $5.48 \mathrm{E}+09$ & $1.83 \mathrm{E}+01$ & $1.71 \mathrm{E}-03$ & $2.64 \mathrm{E}-06$ & $1.74 \mathrm{E}-02$ & $3.48 \mathrm{E}-01$ & 116 & $2.37 \mathrm{E}-09$ \\
\hline $3.16 \mathrm{E}+09$ & $2.52 \mathrm{E}+17$ & $8.39 \mathrm{E}+08$ & $9.74 \mathrm{E}+09$ & $3.25 \mathrm{E}+01$ & $3.03 \mathrm{E}-03$ & $3.88 \mathrm{E}-06$ & $1.18 \mathrm{E}-02$ & $2.37 \mathrm{E}-01$ & 79 & $3.48 \mathrm{E}-09$ \\
\hline $1.00 \mathrm{E}+10$ & $7.96 \mathrm{E}+17$ & $2.65 \mathrm{E}+09$ & $1.73 \mathrm{E}+10$ & $5.78 \mathrm{E}+01$ & $5.39 \mathrm{E}-03$ & $5.69 \mathrm{E}-06$ & $8.07 \mathrm{E}-03$ & $1.61 \mathrm{E}-01$ & 54 & $5.11 \mathrm{E}-09$ \\
\hline $3.16 \mathrm{E}+10$ & $2.52 \mathrm{E}+18$ & $8.39 \mathrm{E}+09$ & $3.08 \mathrm{E}+10$ & $1.03 \mathrm{E}+02$ & $9.59 \mathrm{E}-03$ & $8.36 \mathrm{E}-06$ & $5.50 \mathrm{E}-03$ & $1.10 \mathrm{E}-01$ & 37 & $7.51 \mathrm{E}-09$ \\
\hline $1.00 \mathrm{E}+11$ & $7.96 \mathrm{E}+18$ & $2.65 \mathrm{E}+10$ & $5.48 \mathrm{E}+10$ & $1.83 \mathrm{E}+02$ & $1.71 \mathrm{E}-02$ & $1.23 \mathrm{E}-05$ & $3.75 \mathrm{E}-03$ & $7.49 \mathrm{E}-02$ & 25 & $1.10 \mathrm{E}-08$ \\
\hline $3.16 \mathrm{E}+11$ & $2.52 \mathrm{E}+19$ & $8.39 \mathrm{E}+10$ & $9.74 \mathrm{E}+10$ & $3.25 \mathrm{E}+02$ & $3.03 \mathrm{E}-02$ & $1.80 \mathrm{E}-05$ & $2.55 \mathrm{E}-03$ & $5.10 \mathrm{E}-02$ & 17 & $1.62 \mathrm{E}-08$ \\
\hline $1.00 \mathrm{E}+12$ & $7.96 \mathrm{E}+19$ & $2.65 \mathrm{E}+11$ & $1.73 \mathrm{E}+11$ & $5.78 \mathrm{E}+02$ & $5.39 \mathrm{E}-02$ & $2.64 \mathrm{E}-05$ & $1.74 \mathrm{E}-03$ & $3.48 \mathrm{E}-02$ & 12 & $2.37 \mathrm{E}-08$ \\
\hline $3.16 \mathrm{E}+12$ & $2.52 \mathrm{E}+20$ & $8.39 \mathrm{E}+11$ & $3.08 \mathrm{E}+11$ & $1.03 \mathrm{E}+03$ & $9.59 \mathrm{E}-02$ & $3.88 \mathrm{E}-05$ & $1.18 \mathrm{E}-03$ & $2.37 \mathrm{E}-02$ & 8 & $3.48 \mathrm{E}-08$ \\
\hline $1.00 \mathrm{E}+13$ & $7.96 \mathrm{E}+20$ & $2.65 \mathrm{E}+12$ & $5.48 \mathrm{E}+11$ & $1.83 \mathrm{E}+03$ & $1.71 \mathrm{E}-01$ & $5.69 \mathrm{E}-05$ & $8.07 \mathrm{E}-04$ & $1.61 \mathrm{E}-02$ & 5 & $5.11 \mathrm{E}-08$ \\
\hline $3.16 \mathrm{E}+13$ & $2.52 \mathrm{E}+21$ & $8.39 \mathrm{E}+12$ & $9.74 \mathrm{E}+11$ & $3.25 \mathrm{E}+03$ & $3.03 \mathrm{E}-01$ & $8.36 \mathrm{E}-05$ & $5.50 \mathrm{E}-04$ & $1.10 \mathrm{E}-02$ & 4 & $7.51 \mathrm{E}-08$ \\
\hline $1.00 \mathrm{E}+14$ & $7.96 \mathrm{E}+21$ & $2.65 \mathrm{E}+13$ & $1.73 \mathrm{E}+12$ & $5.78 \mathrm{E}+03$ & $5.39 \mathrm{E}-01$ & 1.23E-04 & $3.75 \mathrm{E}-04$ & $7.49 \mathrm{E}-03$ & 2 & $1.10 \mathrm{E}-07$ \\
\hline
\end{tabular}




\section{Microbunching}

- It appears that the slip factor has to be controlled to $10^{-7}$.

- The natural momentum spread is $\sigma_{\delta}=1.90 \times 10^{-5}$.

If we expand momentum compaction as

$\alpha=\alpha_{0}+\alpha_{1} \delta+\alpha_{2} \delta^{2}+\alpha_{3} \delta^{3}+\cdots$

We must control $\alpha_{0}, \alpha_{1}$ and even $\alpha_{2}$, using quadrupoles, sextupoles, and octupoles, respectively.

- Whether the slip factor can be controlled to such high accuracy remains to be seen. 


\section{Conclusions}

- We would like to produce coherent synchrotron radiation at the 20-m electron storage ring under commission now at ICEEM.

- For coherent radiation of frequency up to $1 \mathrm{THz}$, a cavity-like grating structure can be used.

- The low-energy nature of the ring limits the frequency of such radiation.

- For freq, higher than $1 \mathrm{THz}$, inverse Compton scattering can be used.

- However, for the radiation to reach saturation and remain coherent, the slip factor seen by particles of all momenta in the bunch must be controlled to $|\eta| \lesssim 10^{-7}$.

- This implies the control of momentum compaction up to $\mathcal{O}\left(\delta^{2}\right)$ and is extremely difficult.

- Whether this method works at ICEEM remains an open question. 


\section{Linac}

- Main difference from rings is no synchrotron oscillation because

1. Total linac length is not long enough

2. Bunch is placed at crest of rf wave.

- Thus there is no head-tail exchange.

Tail particles constantly affected by head particles.

- Longitudinal and transverse wake effects will be different from accelerator rings. 


\section{Longitudinal Effects}

- Beam particles lose energy constantly because of radiation.

- But tail particles lose more because of wake left by head particles.

- Example of SLAC linac:

Linac length $L=3 \mathrm{~km}$, cell length $L_{0}=3.5 \mathrm{~cm}$.

Wake: $W_{0}^{\prime}(0)=7.0 \mathrm{~cm}^{-1}$ per cell

$$
W_{0}^{\prime}\left(\sigma_{\ell}\right)=4.5 \mathrm{~cm}^{-1} \text { per cell, } \sigma_{\ell}=1 \mathrm{~mm} \text {. }
$$

- Change from Gaussian units to MKS units:

$W_{0}^{\prime}$ is in stat volts/stat coulomb

1 stat volt $=300 \mathrm{~V}, 1$ stat coulomb $=\frac{1}{3} \times 10^{9}$ coulombs.

Thus $W_{0}^{\prime}(0)=7.0 \times 300 \times 3 \times 10^{9}=6.29 \times 10^{12} \mathrm{~V} / \mathrm{C}$.

- Two particle model: bunch represented by 2 macro-bunches $\sigma_{\ell}$ apart, each carrying charge $\frac{1}{2} \mathrm{eN}$ with $N=5 \times 10^{10}$.

head particle: $\Delta \mathcal{E}_{\text {head }}=-\frac{1}{2} \frac{1}{2} N e^{2} W_{0}^{\prime}(0) \frac{L}{L_{0}}=-1.08 \mathrm{GeV} /$ electron

tail particle: $\Delta \mathcal{E}_{\text {tail }}=-\left[\frac{1}{2} \frac{1}{2} N e^{2} W_{0}^{\prime}(0)+\frac{1}{2} N e^{2} W_{0}^{\prime}\left(\sigma_{\ell}\right)\right] \frac{L}{L_{0}}$

$$
=-1.08-1.39=-2.47 \mathrm{GeV} / \text { electron }
$$




\section{Compensation}

- If uncorrected, energy spread of bunch will increase. Unwanted chromatic effects may result.

- Usually bunch is placed at crest of rf wave to receive maximum acceleration. Can displace it so that head receives less energy than tail.

- SLAC linac: $V_{\mathrm{rf}}=600 \mathrm{kV}$ per cell. For $L=3 \mathrm{~km}$, total $\mathrm{rf}$ $V_{t}=V_{\mathrm{rf}} \frac{L}{L_{0}}=51.4 \mathrm{GV} . \quad \frac{\omega_{\mathrm{rf}}}{2 \pi}=2.8 \mathrm{GHz}$

- RF wave is $V=V_{t} \cos \left(\frac{\omega_{\mathrm{rf}} Z}{c}+\phi\right)$ with $\phi=0$ implying at crest.

- From 2-particle model, $\Delta \mathcal{E}=\Delta \mathcal{E}_{\text {tail }}-\Delta \mathcal{E}_{\text {head }}=-1.39 \mathrm{GeV}$.

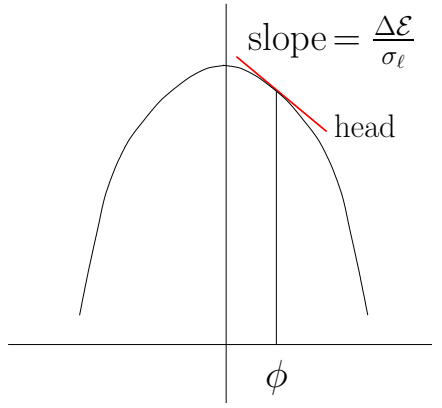

- We choose phase offset $\phi$ so that $V^{\prime}=-V_{t} \sin \phi \frac{\omega r f}{c}=\frac{\Delta \mathcal{E}}{\sigma_{\ell}} \longrightarrow \phi=27.4^{\circ}$.

- Actual computation gives $\Delta \mathcal{E}=-0.9 \mathrm{GeV}$ and $\phi=17.3^{\circ}$. 


\section{Transverse Effects}

- Want to address the effect of transverse wake.

- In a linac, tail particles are constantly pushed sideway by head wake of particles.

- Deflection of tail accumulates along linac.

- When tail particles hit vacuum chamber $\longrightarrow$ beam loss.

- This is called beam breakup (BBU)

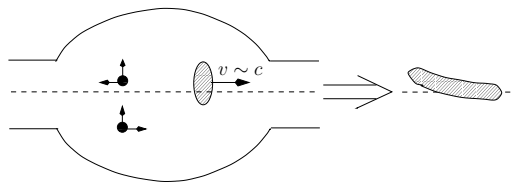

Particles in same vertical slice receive same vertical impulse independent of vertical position. Can lead to beam breakup.

- To avoid BBU, transverse wake must be suppressed. 
- A short bunch: represented by 2 macroparticles each of charge $\frac{1}{2} e N$.

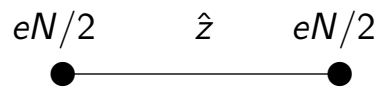

$$
\hat{z}=\text { rms bunch length }
$$

- $\frac{d^{2} y_{1}}{d s^{2}}+k_{\beta_{1}}^{2} y_{1}=0$

$L=$ length of a number of cavities

$\frac{d^{2} y_{2}}{d s^{2}}+k_{\beta_{2}}^{2} y_{2}=-\frac{e^{2} N_{b} W_{1}(\hat{z})}{2 L E_{0}}$
$k_{\beta_{1,2}}=\frac{\nu_{\beta_{1,2}}}{R} \sim \frac{1}{\beta_{\perp}}$ for a ring.

For linac, $\nu_{\beta_{1,2}}$ is number of betatron oscillations in length $L$.

- Solution: $y_{1}(s)=y_{10} \cos k_{\beta_{1}} s$

$y_{2}(s)=y_{10} \cos \bar{k}_{\beta} s \cos \frac{\Delta k_{\beta} s}{2}-y_{10} \sin \bar{k}_{\beta} s\left[\frac{\Delta k_{\beta}}{2}+\frac{e^{2} N_{b} W_{1}(\hat{z})}{4 L E_{0} \bar{k}_{\beta}}\right]\left[\frac{\sin \Delta k_{\beta} s / 2}{\Delta k_{\beta} / 2}\right]$,

with $\bar{k}_{\beta}=\frac{1}{2}\left(k_{\beta_{1}}+k_{\beta_{2}}\right), \quad \Delta k_{\beta}=k_{\beta_{2}}-k_{\beta_{1}}$

- As $\Delta k_{\beta} \rightarrow 0$, last term $\propto s$

- This is a resonance growth. 
- $y_{2}(s)=y_{1}(s)-s y_{10} \sin k_{\beta_{1}} s \frac{e^{2} N_{b} W_{1}(\hat{z})}{4 E_{0} L k_{\beta}}$

Thus in a length $L_{0}$, tail's oscillation amp grows by $\Upsilon_{1}$-fold:

$\Upsilon_{1}=-\frac{e^{2} N_{b} W_{1}(\hat{z}) L_{0}}{4 E_{0} L k_{\beta}}$

- Let us look at the transverse wake. Assuming broadband,

$W_{1}(z)=-\frac{\omega_{r}^{2} Z_{1}^{\perp}}{Q \bar{\omega}} e^{-\alpha z / c} \sin \frac{\bar{\omega} z}{c}$

$Z_{1}^{\perp}$ is transverse impedance at the resonant frequency $\omega_{r}$

$\bar{\omega}=\sqrt{\omega_{r}^{2}-\alpha^{2}}$

$\alpha=\omega_{r} /(2 Q), Q$ being the quality factor.

- Introduce dimensionless variables

$v=\frac{\omega_{r} \sigma_{\ell}}{c}, \quad t=\frac{z}{\sigma_{\ell}}$, and $\phi=v t \cos \phi_{0}=\frac{\bar{\omega} z}{c}$

with $\cos \phi_{0}=\sqrt{1-\frac{1}{4 Q^{2}}}$ or $\sin \phi_{0}=\frac{1}{2 Q}$

- For $Q>\frac{1}{2}$

$\left.W_{1}\right|_{\text {min }}=-2 \omega_{r} Z_{1}^{\perp} \tan \phi_{0} \cos \phi_{0} e^{-\left(\frac{\pi}{2}-\phi_{0}\right) \tan \phi_{0}}$ 
- Minimum at $\left.W_{1}\right|_{\min }=-2 \omega_{r} Z_{1}^{\perp} \tan \phi_{0} \cos \phi_{0} e^{-\left(\frac{\pi}{2}-\phi_{0}\right) \tan \phi_{0}}$ at $\phi=\frac{\pi}{2}-\phi_{0}$ or $\frac{\alpha z}{c}=\left(\frac{\pi}{2}-\phi_{0}\right) \tan \phi_{0}$.

Plot of dipole wake $W_{1}$ with $\omega_{r}=50 \mathrm{GHz}$

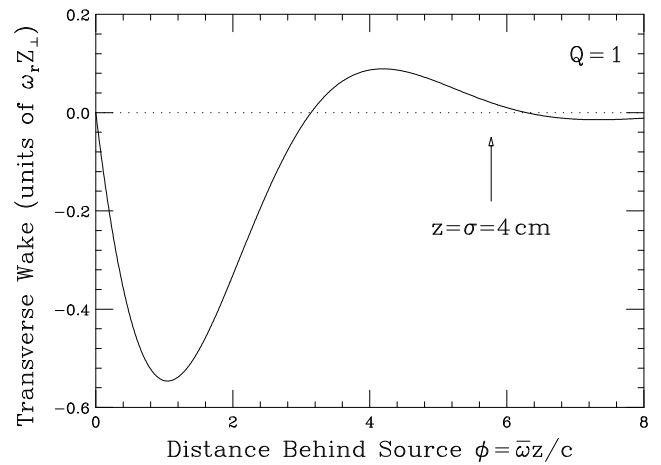

- 2-particle model applicable only in linear part of wake, usually for only short bunches.

- Or valid only when $\phi=\frac{\bar{\omega} z}{c} \ll 1 \quad \longrightarrow \quad \sigma_{\ell} \ll \frac{1}{2} \frac{\lambda}{2 \pi}$

- With $Q \sim 1$, resonant freq $f_{r}=7.96 \mathrm{GHz}\left(\omega_{r}=50 \mathrm{GHz}\right)$, two-particle model works only when the rms bunch length $\sigma_{\ell} \ll 3 \mathrm{~mm}$. 


\section{Long Bunches}

- Although long bunches are for proton or muon rings, will go over theory because it will be useful later.

- For linear density $\lambda(z)$, transverse motion of a particle is $\frac{d^{2} y(z, s)}{d s^{2}}+k_{\beta}^{2} y(z, s)=-\frac{e^{2} N_{b}}{L E_{0}} \int_{-\infty}^{z} d z^{\prime} \lambda\left(z^{\prime}\right) W_{1}\left(z-z^{\prime}\right) y\left(z^{\prime}, s\right)$.

- One solution is by iteration: equate LS to zero and solve for $y(z, s)$. substitute soln on RS, and solve again, iterate until soln is stable.

- When amplitude growth factor $\Upsilon_{1}$ is large, soln gives growth as powers of $\Upsilon_{1}$ or even exponential.

- Soln very sensitive to $\left[\beta_{y} Z_{1}^{\perp}\right], \omega_{r}$, as well as $Q$. 
- Beam-breakup growth for 1000 turns of a muon bunch of intensity $4 \times 10^{12}$ at $50 \mathrm{GeV}$ interacting with a broadband impedance of $Q=1$, $Z_{1}^{\perp}=0.1 \mathrm{M} \Omega / \mathrm{m}$ at the angular resonant frequency of $\omega_{\mathrm{r}}=50 \mathrm{GHz}$.

- Left: rms 13-cm bunch has total growths of 32.50, 7.4, 2.0, 1.09, 1.006, respectively for $\left\langle\beta_{y}\right\rangle=30,25,20,15,10 \mathrm{~m}$.

Right: rms 4-cm bunch has total growths of $29713,3361,287,16.2$, respectively for $\left\langle\beta_{y}\right\rangle=25,20,15,10 \mathrm{~m}$.
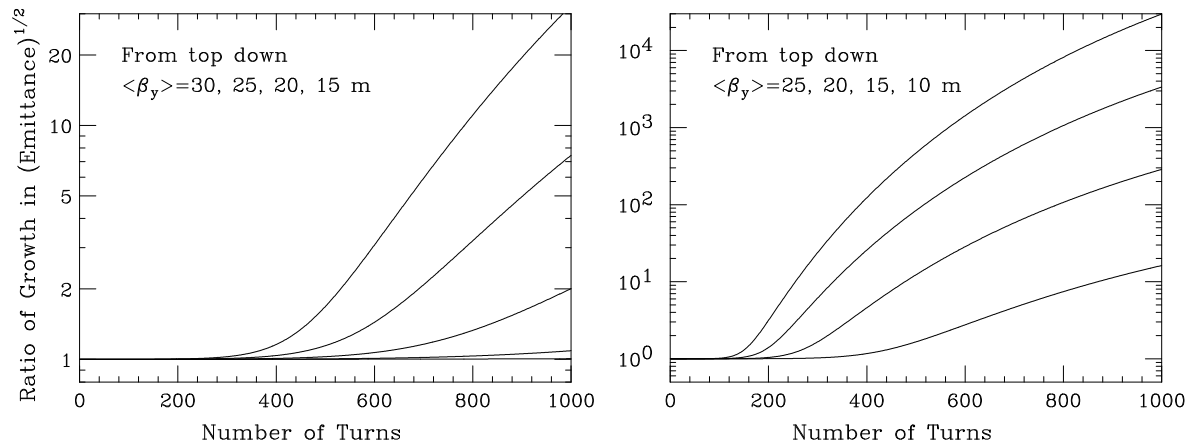


\section{Balakin-Novokhatsky-Smirnov Damping (BNS)}

- Kim, Wurtele, and Sessler suggested to suppress beam breakup by a small tune spread in the beam, coming either through chromaticity, amplitude dependency, or beam-beam interaction. [35]

- A beam particle will be resonantly driven by only a small number of particles in front that have the same betatron tune.

This is a form of Balakin-Novokhatsky-Smirnov (BNS) damping suggested in 1983. [36]

- To implement this, add a detuning term $\Delta \nu_{\beta_{i}}=a\left[y_{i}^{2}+\left(\left\langle\beta_{y}\right\rangle y_{i}^{\prime}\right)^{2}\right]$ to the ith particle, as if it is contributed by an octupole or sextupole.

- The rms tune spread becomes $\sigma_{\nu_{\beta}}=a\left\langle\sigma_{y}^{2}+\left(\left\langle\beta_{y}\right\rangle \sigma_{y^{\prime}}\right)^{2}\right\rangle$.

- Continue the example of a muon bunch of intensity $4 \times 10^{12}$. Solve for the growth in 1000 turns, assuming $\left\langle\beta_{\perp}\right\rangle=20 \mathrm{~m}$ and $Z_{1}^{\perp}=0.1 \mathrm{M} \Omega / \mathrm{m}$. 
- Left: growths of rms $13 \mathrm{~cm}$ bunch are 1.36, 1.08, 1.02, 1.007 for rms tune spread of $\sigma_{\nu_{\beta}}=0.0002,0.0004,0.0006,0.0008$.

Right: growths of rms $4 \mathrm{~cm}$ bunch are 1.58, 1.23, 1.08, 1.03, 1.012 for rms tune spread of $\sigma_{\nu_{\beta}}=0.002,0.003,0.004,0.005,0.006$.
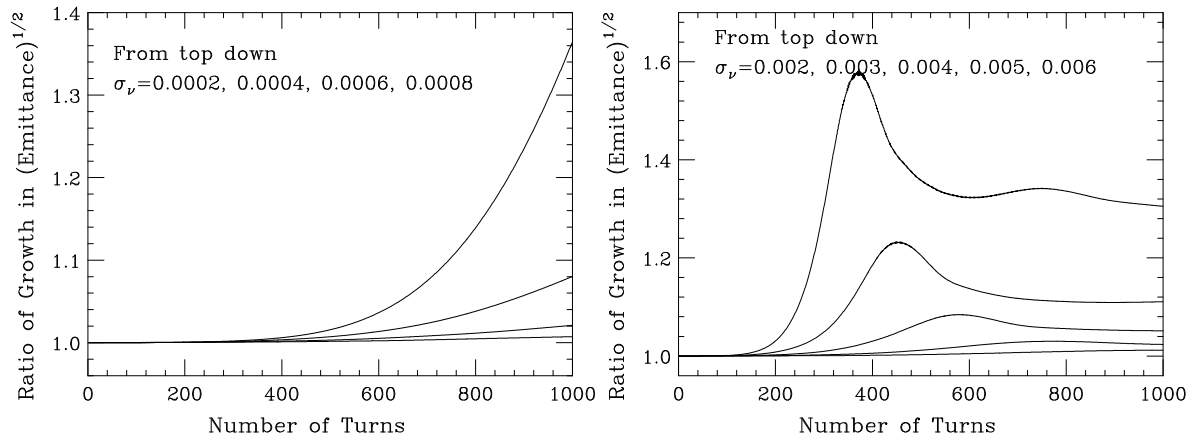

- Notice that for $13-\mathrm{cm}$ bunch, $\sigma_{\nu_{\beta}}=0.0006$ damps growth to $<1.08$. To do the same for 4-cm bunch, $\sigma_{\nu_{\beta}}=0.004$ is needed.

- Thus BNS damping is good for long bunch only. 


\section{Autophasing [37]}

- Let us look at the result of two-particle model again.

$y_{2}(s)=y_{10} \cos \bar{k}_{\beta} s \cos \frac{\Delta k_{\beta} s}{2}-y_{10} \sin \bar{k}_{\beta} s\left[\frac{\Delta k_{\beta}}{2}+\frac{e^{2} N_{b} W_{1}(\hat{z})}{4 L E_{0} \bar{k}_{\beta}}\right]\left[\frac{\sin \Delta k_{\beta} s / 2}{\Delta k_{\beta} / 2}\right]$,

- If we let the tune difference $\Delta k_{\beta}=-\frac{e^{2} N_{b} W_{1}(\hat{z})}{2 L E_{0} \bar{k}_{\beta}}$,

the resonant growth term will be eliminated.

Then $y_{2}(s)=y_{10} \cos \bar{k}_{\beta} s \cos \frac{\Delta k_{\beta} s}{2}$

- This implies we allow a tune difference along the linear bunch density.

- Can do better by letting $\Delta k_{\beta}=-\frac{e^{2} N_{b} W_{1}(\hat{z})}{4 L E_{0} \bar{k}_{\beta}}=\frac{\Upsilon_{1}}{L_{0}}$

Then $y_{2}(s)=y_{10}\left[\cos \bar{k}_{\beta} s \cos \frac{\Delta k_{\beta} s}{2}-\sin \bar{k}_{\beta} s \sin \frac{\Delta k_{\beta} s}{2}\right]=y_{10} \cos k_{\beta 1} s$ exactly same as head particle.

- Being in phase all the time, the tail cannot be driven by the head at all. This is another form of BNS damping known as autophasing. 


\section{Autophasing for Long Bunches}

- Add $\Delta k_{\beta}$ to $k_{\beta}$ to get

$$
\frac{d^{2} y(z, s)}{d s^{2}}+\left[k_{\beta}+\Delta k_{\beta}(z)\right]^{2} y(z, s)=-\frac{e^{2} N_{b}}{L E_{0}} \int_{-\infty}^{z} d z^{\prime} \lambda\left(z^{\prime}\right) W_{1}\left(z-z^{\prime}\right) y\left(z^{\prime}, s\right)
$$

$\lambda(z)$ is linear bunch density.

- In order to have $y(z, s) \sim \sin \left(k_{\beta} s+\varphi_{0}\right)$ independent of $z$, one needs $2 k_{\beta} \Delta k_{\beta}+\Delta k_{\beta}^{2}(z)=-\frac{e^{2} N_{b}}{L E_{0}} \int_{-\infty}^{z} d z^{\prime} \lambda\left(z^{\prime}\right) W_{1}\left(z-z^{\prime}\right)$

- For small compensation, $\frac{\Delta k_{\beta}(z)}{k_{\beta}}=-\frac{e^{2} N_{b} R}{2 L E_{0} k_{\beta}^{2}} \int_{-\infty}^{z} d z^{\prime} \lambda\left(z^{\prime}\right) W_{1}\left(z-z^{\prime}\right)$

- For Gaussian $\lambda(z)$ and resonant wake, can integrate to close form in terms of complex error function.

- Continue with example of muon bunches. 
- For long bunches like muon bunches $v=\omega_{r} \sigma_{\ell} / c=6.67$ and 21.7, compensation is mostly symmetric and Gaussian.

- For short electron bunches, compensation becomes linear. Can implement thru chromaticity by displacing bunch from crest.
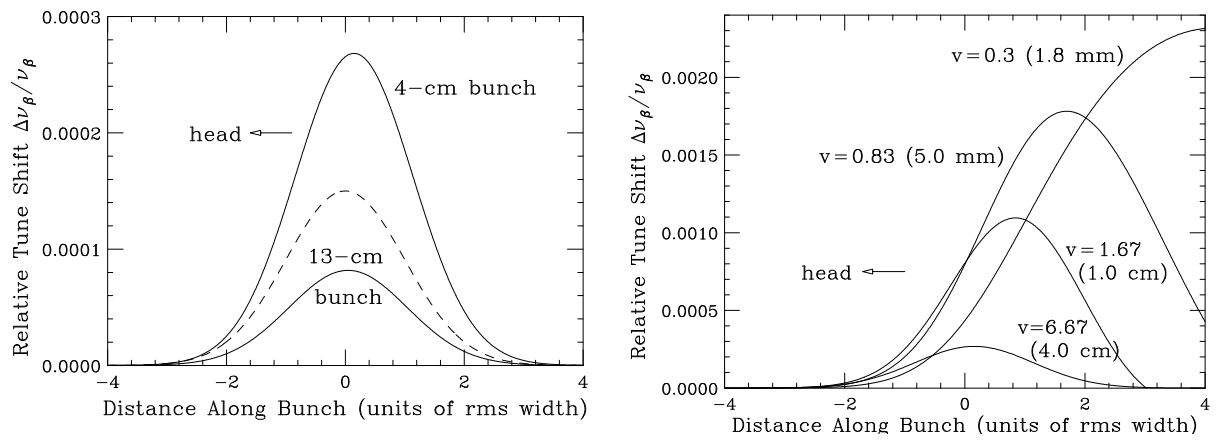

- Autophasing is used mostly for short electron bunches in linac.

- For long bunches in a ring, need rf quadrupole to be pulsed according to compensation curve as bunch passing through.

- If frequency is high, one needs cavities having dipole oscillations. 


\section{Adiabatic Damping in Linacs}

- In linacs, beam energy increases rapidly, 2-particle model should read:

$$
\begin{aligned}
& \frac{1}{\gamma} \frac{d}{d s}\left(\gamma \frac{d y_{1}}{d s}\right)+k_{\beta}^{2} y_{1}=0 \\
& \frac{1}{\gamma} \frac{d}{d s}\left(\gamma \frac{d y_{2}}{d s}\right)+k_{\beta}^{2} y_{2}=-\frac{e^{2} N_{b} W_{1}(\hat{z})}{2 L \gamma E_{\text {rest }}} y_{1}
\end{aligned}
$$

- Assume linear acceleration $\gamma(s)=\gamma_{i}(1+\alpha s), \quad \alpha$ constant.

- First equation becomes $\frac{d}{d u}\left(u \frac{d y_{1}}{d u}\right)+\frac{k_{\beta}^{2}}{\alpha^{2}} u y_{1}=0$, with $u=1+\alpha s$.

Solution is $y_{1}(s)=\hat{y} J_{0}\left[k_{\beta}(1+\alpha s) / \alpha\right] \approx \frac{\hat{y}}{\sqrt{1+\alpha s}} \cos k_{\beta} s$, since $\alpha / k_{\beta} \ll 1$ usually.

SLAC linac, $E_{i}=1 \mathrm{GeV}$ to $E_{f}=50 \mathrm{GeV}, \alpha=0.0163 \mathrm{~m}^{-1}$, while the betatron wave number is $k_{\beta}=0.06 \mathrm{~m}^{-1}$.

- Tail particle: $\frac{d}{d u}\left(u \frac{d y_{2}}{d u}\right)+\frac{k_{\beta}^{2}}{\alpha^{2}} u y_{2}=-\frac{e^{2} N_{b} W_{1}(\hat{z})}{2 L E_{i} \alpha^{2}} \frac{\hat{y}}{\sqrt{u}} \cos k_{\beta} s$

- Try $y_{2}=\frac{D \sin k_{\beta} s}{\sqrt{u}}$ with $D$ a slowly varying function of $u$. 
- Get $y_{2}(s)=\frac{\hat{y}}{\sqrt{1+\alpha s}}\left[\cos k_{\beta} s-\frac{e^{2} N_{b} W_{1}(\hat{z})}{4 L E_{i} \alpha k_{\beta}} \ln (1+\alpha s) \sin k_{\beta} s\right]$.

- Since $E_{f}=E_{i}\left(1+\alpha L_{0}\right), \alpha L_{0} \approx \frac{E_{f}}{E_{i}}$.

- Growth for the whole length $L_{0}$ is $\Upsilon_{1}=-\frac{e^{2} N_{b} W_{1}(\hat{z}) L_{0}}{4 k_{\beta} E_{f} L} \ln \frac{E_{f}}{E_{i}}$.

- Compare with former result, there is an extra factor of $\mathcal{F}=\frac{E_{i}}{E_{f}} \ln \frac{E_{f}}{E_{i}}$.

- For SLAC linac, $\mathcal{F}=1 / 12.8=0.0782$ meaning that the tail will be deflected by 12.8 less with the acceleration.

- This effect is called adiabatic damping. 


\section{Detuned Cavities}

- We shown before that a spread in betatron tune helps in alleviating bbu. Such spread can come from a detuned cavity structure.

- Dipole wake of a cavity structure is given by

$$
W_{1}(z)=-2 \sum_{n} K_{n} \sin \frac{2 \pi \nu_{n} z}{c} e^{-\pi \nu_{v} z /\left(c Q_{n}\right)} \quad z>0
$$

where $K_{n}=\frac{n_{n} c}{Q_{n}}, \nu_{n}$, and $Q_{n}$ are kick factor, resonant frequency, and quality factor of the $n$th eigenmode in the structure.

- To reduce bbu, it is important to reduce this dipole wake $W_{1}(z)$.

- One way to reduce $W_{1}(z)$ is to manufacture the cavity structure with cell dimension varying gradually

so that each cell has a slightly different resonant frequency.

- Effect of the wake due to sharp resonant peak of each individual cell will not add together and wake of the whole structure will be reduced.

- Such a structure is called a detuned cavity structure. 


\section{Short-Range Part of Wake}

- For short range, can assume all cells do not couple.

Total wake is sum of wake of individual cells.

- Cell-to-cell variation is small, can replace sum over cells by an integral. $W_{1}(z) \approx-2 \int d \nu K \frac{d n}{d \nu} \sin \frac{2 \pi \nu z}{c} \quad$ one eigenmode included only

- $W_{1}(z)$ defined as dipole wake per cell $\longrightarrow \frac{d n}{d \nu}$ normalized to unity.

- Let $\nu=\bar{\nu}+x$, where $\bar{\nu}$ is average resonant frequency.

$$
\begin{aligned}
& W_{1}(z) \approx-2 \operatorname{Im}[e_{\uparrow}^{2 i \pi \bar{\nu} z / c} \underbrace{\int d x K(\bar{\nu}+x) \frac{d n}{d \nu}(\bar{\nu}+x) e^{2 \pi i x z / c}}_{\uparrow}] \\
& \text { rapidly varying slowly varying } \\
& \text { envelope }
\end{aligned}
$$

- Slowly varying part or envelope is Fourier transform of $K \frac{d n}{d \nu}$. 
Two Examples

- Uniform frequency distribution with full frequency spread $\Delta \nu$ :

$W_{1}(z) \approx-2 \bar{K} \sin \frac{2 \pi \bar{\nu} z}{c} \frac{\sin (\pi \Delta \nu z / c)}{\pi \Delta \nu z / c}$

- Gaussian freq distribution with rms width $\sigma_{\nu}$ :

$W_{1}(z) \approx-2 \bar{K} \sin \frac{2 \pi \bar{\nu} z}{c} e^{-2\left(\pi \sigma_{\nu} z / c\right)^{2}}$

Rapid decay as Gaussian and is therefore preferred.

Next Linear Collider (NLC)

$N=206$ cells

$\bar{\nu}=15.25 \mathrm{GHz}$

$\bar{K}=40 \mathrm{MV} / \mathrm{nC} / \mathrm{m}^{2}$

Detuned distribution:

Gaussian $\pm 2.5 \sigma_{\nu}$

$\sigma_{\nu}=2.5 \%$ of $\bar{\nu}$.

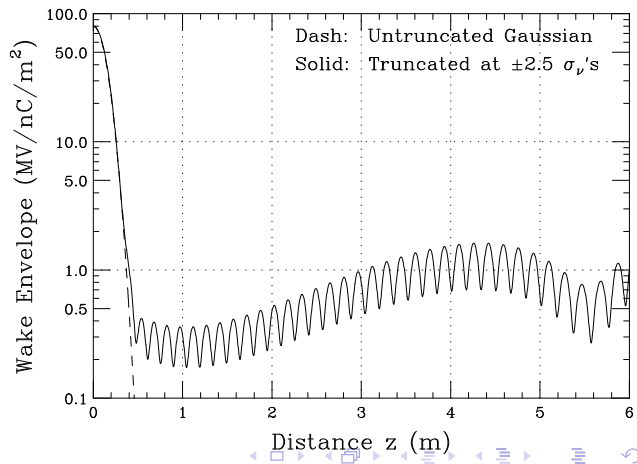




\section{Comments on NLC Detuned Wake}

- The negative signs in the wake expressions are just convention.

- The detuned dipole wake actually starts from zero, increases linearly, reaches a maximum, and rolls off like a Gaussian.

- For larger distance, the roll-off stops, because the detuning is not a true Gaussian, but truncated. So we get $\frac{\sin x}{x}$-behavior.

- There are the $42-\mathrm{cm}$ and $82-\mathrm{cm}$ bunch-spacing scenarios. The dipole wake at the 2 nd bunch has been suppressed by more than 2 orders of magnitude.

- This detuning method is very useful for long-range bunch-to-bunch bbu, but not useful for single bunch bbu.

- However, for long-range suppression, we cannot trust the above result, because cell-to-cell interaction has been neglected. 


\section{Circuit Model for DDS}

- To incorporate interaction between cells, Bane and Gluckstern devised an equivalent circuit to represents cell structure. [38]

- Later Kroll and Jones et al. improved the model by introducing two circuits together with 4 damping manifolds, corresponding to 4 holes in the cells to carry away dipole wake. [39]

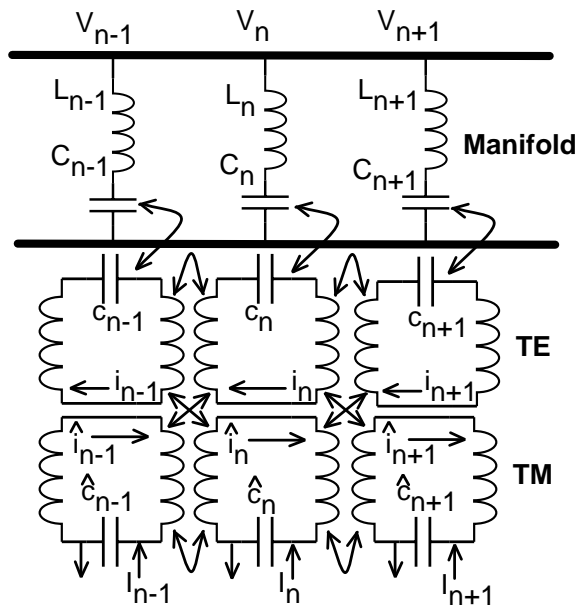

- There are $N=206$ TE and TM resonant circuits connected together.

- The wake is computed by solving a $618 \times 618$ matrix. But the matrix is sparse, which makes solution much easier. 

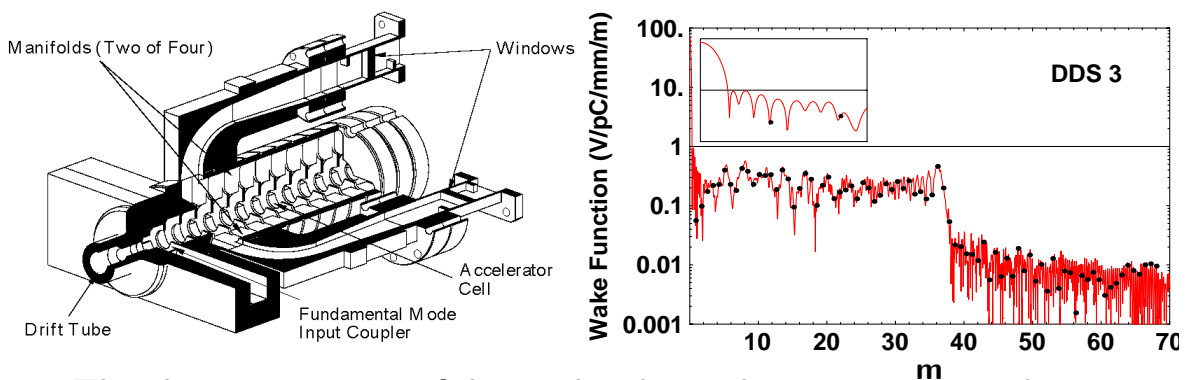

- The short-range part of detuned wake is almost same as earlier calculation.

- The long-range part of detuned wake is suppressed to less than $1 \mathrm{~V} / \mathrm{pC} / \mathrm{mm} / \mathrm{m}$.

- The dots represent the 82 bunches with $84-\mathrm{cm}$ spacing.

- The wake was computed in the frequency domain and Fourier transformed to time domain.

Thus very short-range part may not be accurate.

- Parameters used are obtained by fitting to measured freq vs phase advance dispersion curve. 


\section{Multi-Bunch BBU}

- DDS suppresses the dipole wake by more than 2 orders of magnitude. For the 95-bunch 42-cm scenario of NLC, dipole wake per unit length is only $\sim 0.21 \mathrm{MV} / \mathrm{nC} / \mathrm{m}^{2}$.

- Want to examine how much emittance growth and bbu will be driven by detuned wake, and how much energy spread is required for further suppression.

- 2-particle model is used by considering each bunch as a maga particles 1st bunch: $\frac{d^{2} y_{1}}{d s^{2}}+k_{\beta}^{2} y_{1}=0$

2nd bunch: $\frac{d^{2} y_{2}}{d s^{2}}+k_{\beta}^{2} y_{2}=-\frac{e^{2} N_{b} W_{1}(\hat{z})}{L E} y_{1}$

where $L$ is cavity length and $\hat{z}$ is bunch spacing.

- Soln: 1st bunch $y_{1}(s)=\operatorname{Re} \hat{y} e^{i k_{\beta} s}$

$$
\text { 2nd bunch } y_{2}(s)=\operatorname{Re} \hat{y} \Gamma s e^{i k_{\beta} s} \quad \Gamma=\frac{i e^{2} N_{b} W_{1}(\hat{z})}{2 k_{\beta} L E_{0}}
$$

Keep only particular solution because it increases with $s$. 
- 3rd bunch: $\frac{d^{2} y_{3}}{d s^{2}}+k_{\beta}^{2} y_{3}=-\frac{e^{2} N_{b} W_{1}(2 \hat{z})}{L E_{0}} y_{1}-\frac{e^{2} N_{b} W_{1}(\hat{z})}{L E_{0}} y_{2}$

- Keeping only the most divergent term, i.e., the last term, $y_{3}(s)=\operatorname{Re} \hat{y} \frac{1}{2} \Gamma^{2} s^{2} e^{i k_{\beta} s}$

- Continuing, get $y_{m}(s)=\operatorname{Re} \hat{y} \frac{\Gamma^{m-1} s^{m-1}}{(m-1) !} e^{i k_{\beta} s}$

- If we employ BNS damping on 2nd bunch, amount of tune spread is $\frac{\Delta k_{\beta}}{k_{\beta}}=-\frac{e^{2} N_{b} W_{1}(\hat{z})}{2 k_{\beta}^{2} E_{f}} \ln \frac{E_{f}}{E_{i}}$

where adiabatic damping has been included.

- To damp $n_{b}$ bunches, it is reasonable to assume $n_{b}$ times of spread.

- Tune spread can come from chromaticity.

For FODO lattice of phase advance $\mu$, natural chromaticity is $\xi_{N}=-\frac{2}{\pi} \tan \frac{\mu}{2}$

- If we take $\xi_{N}=-1$, we get required energy spread of $2.7 \%$ 
- Simulation has been performed by Stupakov, assuming initial bunch offset of $1 \mu \mathrm{m}$. [41]

- The vertical emittance of the last or 95th bunch was monitored.

Increase of vertical emittance of 95th bunch:

curve 1: w/o energy variation

curve 2 : $0.8 \%$ energy variation

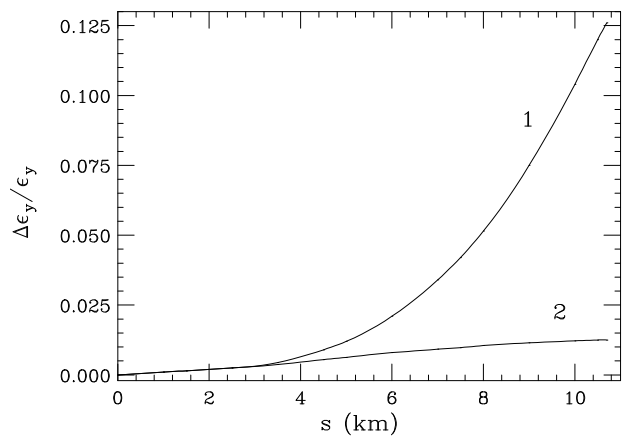

- At $0.8 \%$ energy variation, vertical emittance of 95 th bunch increases by only $1.2 \%$.

- Analytic treatment of multi-bunch bbu has been performed by Bohn, Delayen, and $\mathrm{Ng}$, with good agreement with simulations. [42, 43, 44] 


\section{References}

[1] A.W. Chao, Physics of Collective Beam Instabilities in High Energy Accelerators, John Wiley \& Sons, 1993.

[2] W.K.H. Panofsky and W.A. Wenzel, Rev. Sci. Instrum. 27, 961 (1956).

[3] J. E. Griffin, K. Y. Ng, Z. B. Qian and D. Wildman, Experimental Study of Passive Compensation of Space Charge Potential Well Distortion at the Los Alamos National laboratory Proton Storage Ring, Fermilab Report FN-661, 1997; M. A. Plum, D. H. Fitzgerald, J. Langenbrunner, R. J. Macek, F. E. Merrill, F. Neri, H. A. Thiessen, P. L. Walstrom, J. E. Griffin, K. Y. Ng, Z. B. Qian, D. Wildman and B. A. Jr. Prichard, Phys. Rev. ST Accel. Beams 2, 064201 (1999).

[4] K.Y. Ng, Space-Charge Impedances of Beams with Non-uniform Transverse Distribution, Fermilab Report FN-0756, 2004.

[5] J. Gareyte, Impedances: Measurements and Calculations for Non-symmetric Structures, Proc. EPAC 2002 (Paris, June 3-7, 2002), p. 89. 
[6] K. Y. Ng, Space-Charge Impedances of Beams with Non-uniform Transverse Distributions, Fermilab Report FN-0756, 2004.

[7] L. D. Landau, J. Phys. USSR 10, 25 (1946).

[8] J. D. Jackson, Nucl. Energy Part C: Plasma physics 1, 171 (1960).

[9] K. Y. Ng, Decoherence and Landau Damping, Fermilab Report FERMILAB-FN-0763-AD, 2005.

[10] A. W. Chao, Physics of Collective Beam Instabilities in High Energy Accelerators, Wiley Interscience, 1993, Chapter 5.

[11] K.Y. Ng, Longitudinal Instabilities and Stability Criteria, Physics of Particle Accelerators, AIP Conf. Proc. 184, ed. M. Month and M. Dienes, 1888, p.1989; Fermilab Report FN0438, 1986.

[12] E. Keil and W. Schnell, CERN Report TH-RF/69-48 (1969); V. K. Neil and A. M. Sessler, Rev. Sci. Instrum. 36, 429 (1965).

[13] A. Hofmann, ISR Performance report, CERN Report, August 17, 1976. 
[14] D. Boussard, CERN Report CERN-LAB II/RF/Int./75-2 (1975).

[15] F. J. Sacherer, A Longitudinal Stability Criterion for Bunched Beams, CERN Report CERN/MPS/BR 73-1, 1973; IEEE Trans. Nucl. Sci. NS 20(3), 825 (1973).

[16] P. B. Robinson, Stability of Beam in Radiofrequency System, Cambridge Electron Accel. Report CEAL-1010, 1964.

[17] K.Y. Ng, Longitudinal Instabilities and Stability Criteria of the Fermilab Main Ring, AIP Conference Proc. 184, Physics of Particle Accelerators, ed. M. Month and M. Dienes, AIP, New York, 1989, Vol. 2, p.1969.

[18] F. J. Sacherer, Methods for Computing Bunched-Beam Instabilities, CERN Report CERN/SI-BR/72-5, 1972.

[19] See for example, J. L. Laclare, Bunch-Beam Instabilities, - Memorial Talk for F. J. Sacherer, Proc. 11th Int. Conf. High-Energy Accel., (Geneva, July 7-11, 1980), p. 526. 
[20] B. Zotter and F. Sacherer, Transverse Instabilities of Relativistic Particle Beams in Accelerators and Storage Rings, Proc. First Course of Int. School of Part. Accel. of the 'Ettore Majorana' Centre for Scientific Culture, eds. A. Zichichi, K. Johnsen, and M. H. Blewett (Erice, Nov. 10-22, 1976), CERN Report CERN 77-13, p. 175.

[21] A. Hofmann, Landau Damping, CERN Accelerator School 5th Advanced Accelerator Physics Course, (Hotel Paradise, Rhodes, Greece, Sept. 20-Oct. 1, 1993), p. 275, CERN Report CERN 95-06.

[22] J. Wei, Longitudinal Dynamics of the Non-Adiabatic Regime on Alternating-Gradient Synchrotrons, PhD Dissertation (SUNY, Stony Brook, 1990).

[23] W. W. Lee and L. C. Teng, Beam-Bunch Length Matching at Transition Crossing, Proc. 8th Int. Conf. High Energy Accel., eds. M. H. Blewett and N. Vogt-Nilsen (CERN, Geneva, Sept. 20-24, 1971), p. 327.

[24] W. Hardt, Gamma-Transition-Jump Scheme of the CPS, Proc. 9th Int. Conf. High Energy Accel. (SLAC, Stanford, May 2-7, 1974), p. 434. 
[25] I. Kourbanis and K. Y. Ng, Transition Crossing in the Fermilab Main Ring, Past and Present, Proc. 1993 Part. Accel. Conf., ed. S. T. Corneliussen (Washington, D.C., May 17-20, 1993), p. 3630.

[26] J. A. MacLachlan, ESME: Longitudinal Phase-Space Particle Tracking-Program Documentation, Fermilab Report TM-1274, 1984.

[27] See for example Ref. 3 or I. Kourbanis and K. Y. Ng, Main Ring Transition Crossing Simulations, Proc. Fermilab III Instabilities Workshop, Fermilab, eds. S. Peggs and M. Harvey (Batavia, U.S., 1990), p. 151.

[28] J. M. Hammersley and D. C. Handscomb, Monte Carlo Methods (Wiley, 1964). [29] Kwang-Je Kim and Zhirong Huang, Physics of High-Gain Free-Electron Lasers, USPAS lecture note, updated 2007.

[30] G.A. Kraft and I.V. Bazarov, 4th Generation Light Source II, ERLs and Thomson Scattering, USPAS lecture note, 2002.

[31] H. Wiedermann, Synchrotron Radiation, SLAC-R-637, 2011. 
[32] W.B. Colson, Phys. Lett. 64A, 190 (1977).

[33] S.Y. Lee, Y.C. Jing, T. Luo, X. Pang, X. Wang, and K.Y. Ng, Diffractive Grating Structure for Coherent Light Source Production, Rev. Sci. Inst. 80, 066102 (2009).

[34] R. Bonifacio, C. Pelligrini and L.M. Narducci, Opt. Comm. 50, 373 (1984).

[35] E.-S. Kim, A, M, Sessler, and J. S. Wurtele, Transverse Instability in a $50 \mathrm{GeV} \times 50 \mathrm{GeV}$ Muon Collider Ring, Proc. 1999 Part. Accel. Conf., eds. A. Luccio and W. Mackay (New York, March 27-April 2, 1999), p. 3057.

[36] V. Balakin, A. Novokhatsky, and V. Smirnov, VLEPP: Transverse Beam Dynamics, Proc. 12th Int. Conf. High Energy Accel. eds. F. T. Cole and R. Donaldson (Fermilab, Batavia, Aug. 11-16, 1983), p. 119.

[37] V. E. Balakin, Proc. Int. Workshop Next Generation Linear Collider, ed. M. Riordan (SLAC, Stanford, Nov. 28-Dec. 9, 1988), p. 55.

[38] K. L. F. Bane and R. L. Gluckstern, Part. Accel. 42, 123 (1993). 
[39] R. Jones, K. Ko, N. M. Kroll, R. H. Miller, and K. A. Thompson, Equivalent Circuit Analysis of the SLAC Damped Detuned Structure, Proc. 5th European Part. Accel. Conf. EPAC'96, ed. S. Myers, R. Pascual, and J. Poole (Sitges, Barcelona, June 10-14, 1996), p. 1292; R. Jones, K. Ko, N. M. Kroll, and R. H. Miller, Spectral Function Calculation of Angle Wakes, Wake Moments, and Misalignment Wakes for the SLAC Damped Detuned Structures (DDS), Proc. 1997 Part. Accel. Conf., eds. M. Comyn, M. K. Craddock, M. Reiser, and J. Thomson (Vancouver, Canada, May 12-16, 1997), p. 551; R. Jones, N.M. Kroll, and R.H. Miller, R.D. Ruth, and J.W. Wang, Advanced Damped Detuned Structure Development at SLAC, ibid., p. 548; M. Dehler, R. M. Jones, N. M. Kroll, R. H. Miller, I. Wilson, and J. W. Wuensch, Design of a $30 \mathrm{GHz}$ Damped Accelerating Structure, ibid., p.518; J.-F. Ostiguy, K.Y. Ng, R.M. Jones, Z. Li, and N.M. Kroll, Comparative Study of Medium Damped and Detuned Linear Accelerator Structures, PAC'01, p.3789.

[40] K. Yokoya, Cumulative Beam Breakup in Large-Scale Linacs, DESY 86-084, ISSN 0418-9833, 1986. 
[41] G. Stupakov, talk given in SLAC-Fermilab Video Conference, September, 1999: G. Stupakov, Effect of Energy Spread in the Beam Train on Beam Breakup Instability, SLAC Report, LLC-0027, 1999.

[42] C. L. Bohn and J. R. Delayen, Phys. Rev. A45, 5964 (1992). "Multibunch Domain B" introduced in this reference is the limit of zero focusing variation away from wake zero-crossing; C. L. Bohn and J. R. Delayen, Cumulative Beam Breakup in Radio-Frequency Linacs, Proc. 1990 Linear Accelerator Conference, Los Alamos National Laboratory Report No. LA-12004-C, (Albuquerque, New Mexico, Sept. 10-14, 1990), p. 306.

[43] C. L. Bohn and K. Y. Ng, Theory and Suppression of Multibunch Beam Breakup in Linear Colliders, Proc. XX Int. Linac Conf., (Monterey, CA, Aug. 21-25, 2000), p. 884; C. L. Bohn and K. Y. Ng, Phys. Rev. Lett. 85, 984 (2000); Preserving High Multibunch Luminosity in Linear Colliders, Fermilab Report FERMILAB-PUB-00-072-T, 2000. Erratum p. 5010.

[44] C. L. Bohn and K. Y. Ng, Phys. Rev. Lett. 85, 984 (2000); Preserving High Multibunch Luminosity in Linear Colliders, Fermilab Report FERMILAB-PUB-00-072-T, 2000. Erratum p. 5010. 